\title{
Needle Exchange Programs to Prevent Hepatitis C Virus Infection in People Who Inject Drugs in Rural Appalachia
}

\author{
Stephen M. Davis
}

Follow this and additional works at: https://researchrepository.wvu.edu/etd

\section{Recommended Citation}

Davis, Stephen M., "Needle Exchange Programs to Prevent Hepatitis C Virus Infection in People Who Inject Drugs in Rural Appalachia" (2018). Graduate Theses, Dissertations, and Problem Reports. 5448. https://researchrepository.wvu.edu/etd/5448

This Dissertation is protected by copyright and/or related rights. It has been brought to you by the The Research Repository @ WVU with permission from the rights-holder(s). You are free to use this Dissertation in any way that is permitted by the copyright and related rights legislation that applies to your use. For other uses you must obtain permission from the rights-holder(s) directly, unless additional rights are indicated by a Creative Commons license in the record and/ or on the work itself. This Dissertation has been accepted for inclusion in WVU Graduate Theses, Dissertations, and Problem Reports collection by an authorized administrator of The Research Repository @ WVU. For more information, please contact researchrepository@mail.wvu.edu. 


\title{
Needle Exchange Programs to Prevent Hepatitis C Virus Infection in People Who Inject Drugs in Rural Appalachia
}

\author{
Stephen M. Davis \\ Dissertation submitted \\ to the School of Public Health \\ at West Virginia University
}

in partial fulfillment of the requirements for the degree of Doctor of Philosophy in Public Health Sciences

\author{
Alfgeir L. Kristjansson, Ph.D., Chair \\ Adam Baus, Ph.D. \\ Danielle Davidov, Ph.D. \\ Melanie Fisher, M.D. \\ Keith Zullig, Ph.D.
}

\section{Department of Social and Behavioral Health Sciences}

\author{
Morgantown, West Virginia \\ 2018
}

Keywords: needle exchange programs; hepatitis C virus;

people who inject drugs; paraphernalia laws; barriers to using clean needles; policing behaviors

Copyright 2018 Stephen M. Davis 


\title{
ABSTRACT
}

\section{Needle Exchange Programs to Prevent Hepatitis C Virus Infection in People Who Inject Drugs in Rural Appalachia}

\author{
Stephen M. Davis
}

The recent opioid epidemic in the rural Appalachian region of the United States has fueled an exponential increase in hepatitis $\mathrm{C}$ virus (HCV) infection among People Who Inject Drugs (PWID). Needle exchange programs (NEP), which provide clean needles in exchange for used needles, can reduce the incidence of HCV among PWIDs. However, the empirical evidence examining NEP effectiveness in the prevention of HCV in this population is mixed, only describes studies conducted in urban areas, and does not address the unique challenges associated with implementing NEPs in rural areas. Furthermore, no studies have examined barriers to using clean needles obtained from NEPs in rural settings. Therefore, there is a critical need to understand the unique context and programmatic challenges of rural NEPs to design and implement successful programs to reduce HCV transmission in this underserved population. The objective of this dissertation was to improve empirical evidence related to NEP efficacy, examine programmatic challenges encountered by rural NEPs, and identify clean needle use barriers unique to rural areas. The rationale underlying this research is that improved understanding of operational and clean needle use barriers will promote successful implementation of NEPs in rural areas. In the first study, a systematic review with meta-analysis was conducted to update the empirical evidence related to NEP prevention of HCV in PWIDs. Findings from this study revealed that the overall impact of NEPs on HCV prevention remains unclear and is complicated by significant heterogeneity between studies. Furthermore, no rural studies were identified in the review. Standardization of population characteristics, intervention components, empirical comparisons, and outcome assessments is suggested to reduce heterogeneity and clarify the empirical contribution of NEPs to HCV prevention. In the second study, a qualitative case study design was employed to examine barriers and facilitators to NEP implementation, ongoing operations, and future sustainability.

Structured interviews with program directors, law enforcement leaders and personnel, and NEP attendees revealed common barriers and facilitators to program operations. Findings from this study indicated that despite broad community support, growing volumes, funding shortages, and the federal government's prohibition on the use of funds to purchase needles threatened program operations. Furthermore, paraphernalia laws created a legal conundrum in the form of criminal sanctions for the possession of needles, which may inadvertently promote needle sharing and disease transmission. In the third study, a mixed methods survey of PWIDs attending two rural, Appalachian programs regarding barriers to using clean needles obtained from the exchange for every injection was employed. Findings from this study revealed that fear of arrest and problems with obtaining clean needles from pharmacies were the most commonly endorsed barriers to clean needle use. The results of this dissertation suggest that NEPs opened in rural Central Appalachia in response to an opioid and 
heroin epidemic have enjoyed robust community support and are viewed as an important infectious diseases prevention mechanism by PWIDs. However, the overall impact of NEPs on preventing HCV in rural PWIDs is unclear and, similar to urban areas of the United States, may be blunted by legal structural influences (i.e.

paraphernalia laws) that impact policing behaviors. Future studies should explore the factors associated with these structural barriers that may prevent the ability of rural PWIDs to use a clean needle during every injection, which, in turn, may minimize the overall efficacy of these programs on HCV prevention. Further research is also needed on the feasibility of implementing the addition of opiate replacement therapy (i.e., methadone, buprenorphine) with NEPs given the strong protective effect on HCV seroconversion observed in several European studies implementing this combined approach consistently over time. 


\section{DEDICATION}

This dissertation is dedicated to my wife, Teresa Kay Adams-Davis, who has walked this difficult journey with me and carried me more than she will ever know. 


\section{ACKNOWLEDGMENTS}

None of this work would have been possible without the guidance and expertise of my dissertation committee. Any positive accolades that may stem from this work should necessarily extend to Drs. Kristjansson, Baus, Davidov, Fisher, and Zullig. In particular, I am indebted to my committee chair and mentor, Dr. Alfgeir Kristjansson, for his mental and spiritual support and guidance throughout this entire process. Additionally, the sound expertise, direction, and advice regarding meta-analytic methods received from Dr. George Kelley greatly promoted the successful completion of study 1 . I would also like to acknowledge the support I received from the needle exchange program directors and staff that made my work in dissertation aims 2 and 3 possible. Finally, I would like to acknowledge the attendees of the two needle exchange programs that took the time to participate in studies 2 and 3. 


\section{TABLE OF CONTENTS}

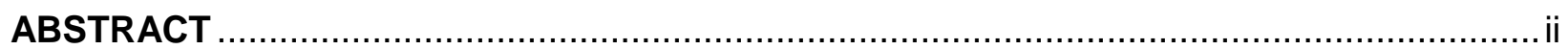

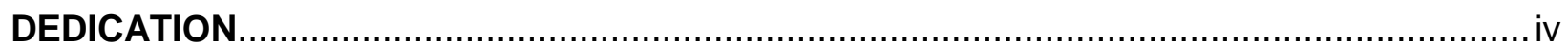

ACKNOWLEDGMENTS

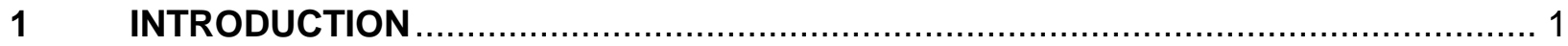

1.1 Hepatitis C Virus Infection in the United States........................................... 1

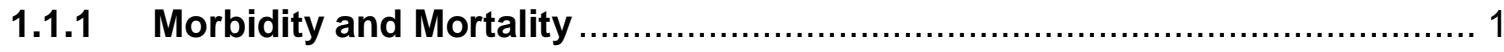

1.1.2 Emerging Epidemic ............................................................................. 1

1.1.2.1 The role of injection drug use and needle sharing ...................................... 2

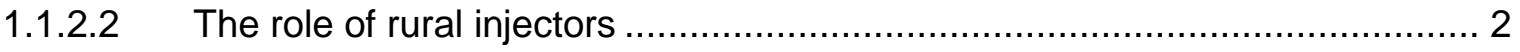

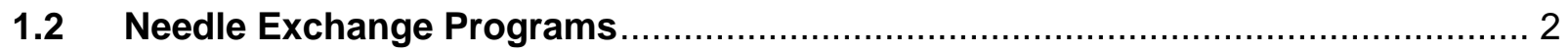

1.2.1 Unclear Efficacy .............................................................................. 3

1.2.2 Barriers to Using Clean Needles and NEP Efficacy ……........................... 3

1.3 Knowledge Gaps and Dissertation Aims

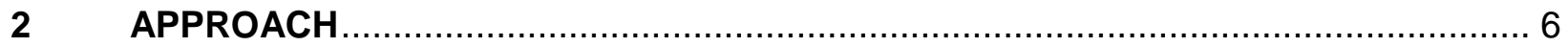

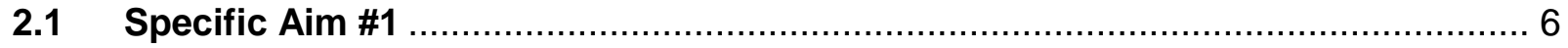

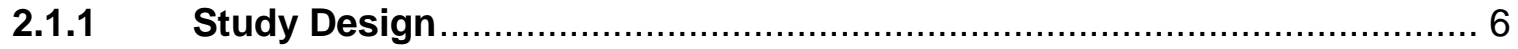

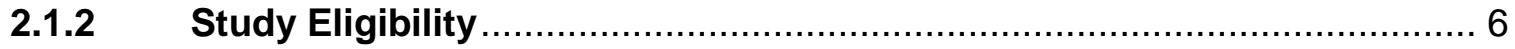

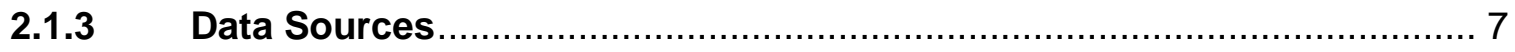

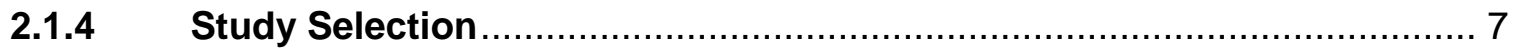

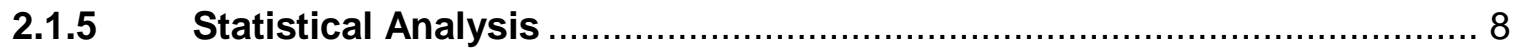

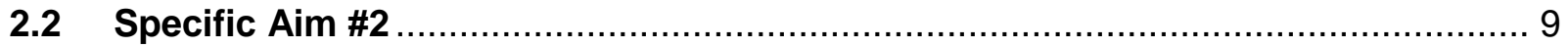

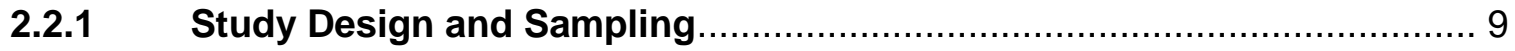

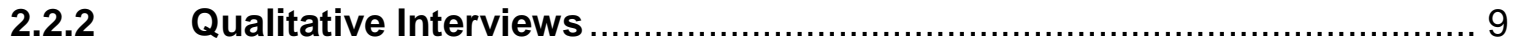

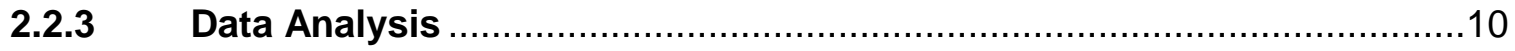

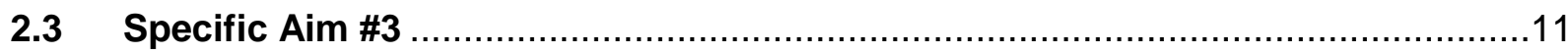

2.3.1 Study Design

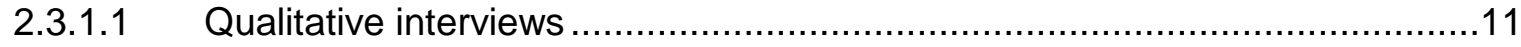

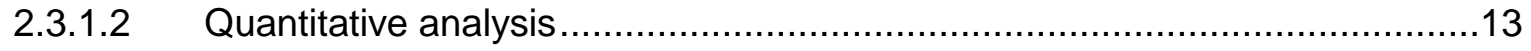

$3 \quad$ MANUSCRIPTS

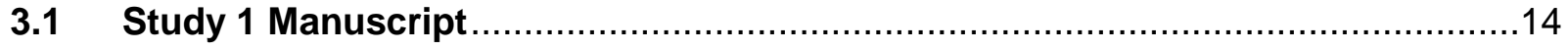

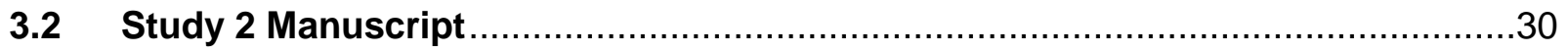

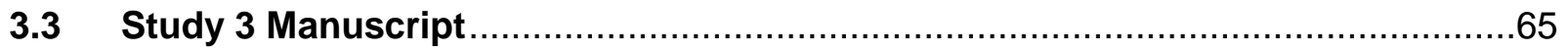

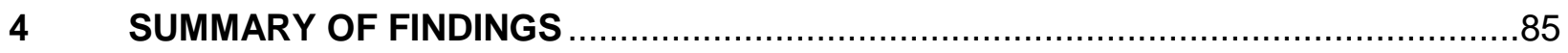




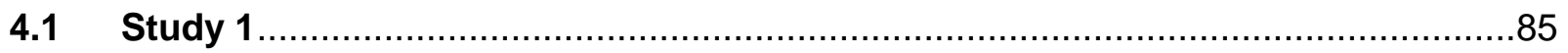

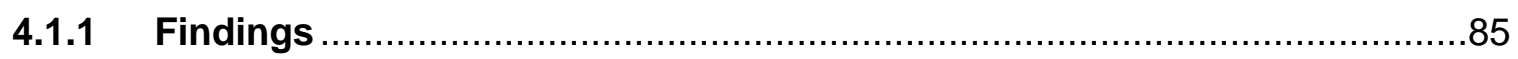

4.1.2 Discussion and Implications for Future Research ............................... 85

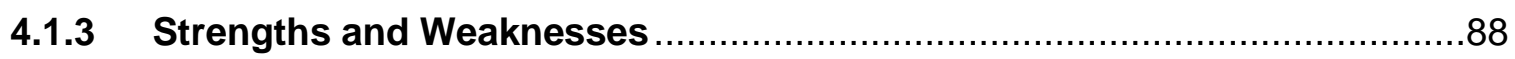

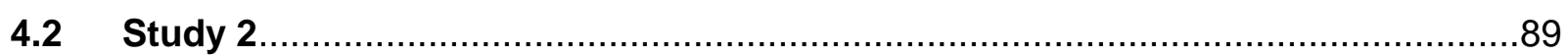

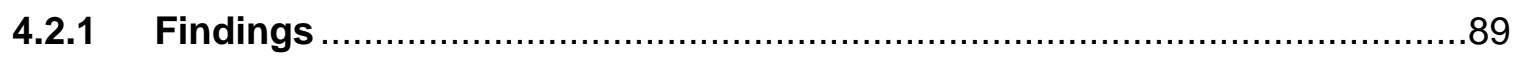

4.2.2 Discussion and Implications for Future Research...............................89

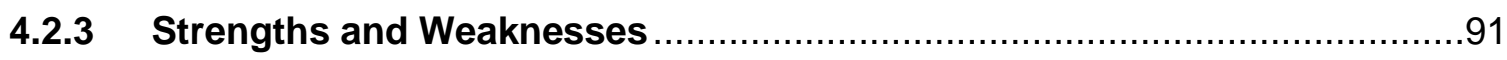

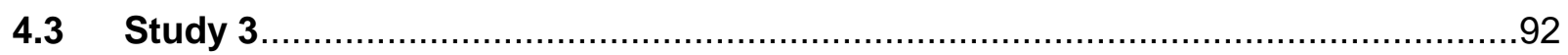

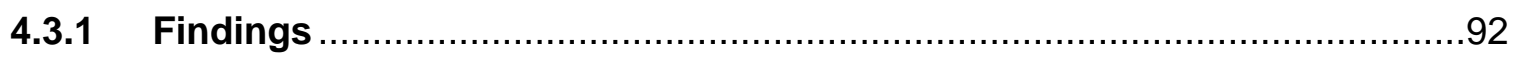

4.3.2 Discussion and Implications for Future Research ..............................92

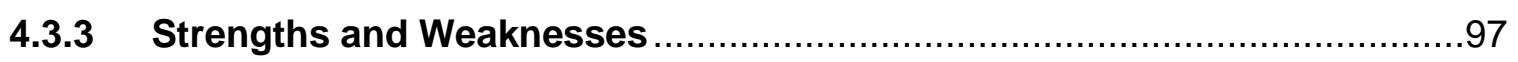

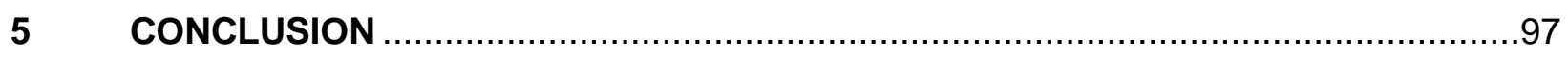

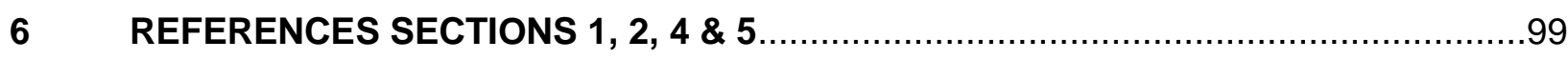

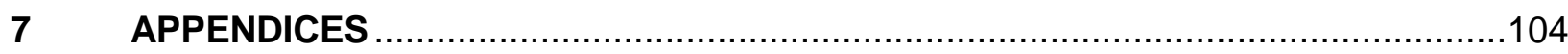

7.1 Study 1 Search Strategy and Results ................................................. 105

7.1.1 PubMed Search Details................................................................... 105

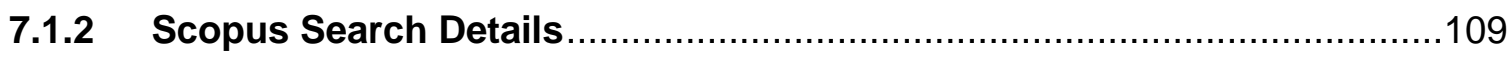

7.1.3 Web of Science Search Details .................................................... 110

7.1.4 CINHAL Search Details ................................................................111

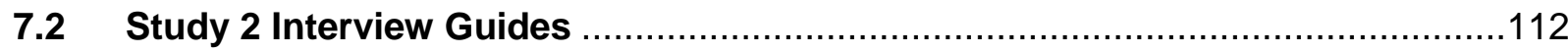

7.2.1 Needle Exchange Program Directors ...........................................112

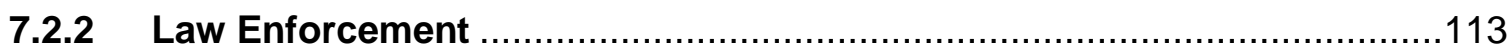

7.2.3 Needle Exchange Program Attendees ............................................114

7.3 Study 3 Barriers to Using New Needles Survey ......................................116 


\section{INTRODUCTION}

\subsection{Hepatitis C Virus Infection in the United States}

\subsubsection{Morbidity and Mortality}

Hepatitis $\mathrm{C}$ virus (HCV) is a blood-borne virus that infects the liver. ${ }^{1}$

Approximately $75 \%$ of acute HCV infections become chronic. ${ }^{2}$ Chronic HCV infection significantly increases the risk of liver disease, especially cirrhosis and liver cancer. ${ }^{2-6}$ Of the approximately 3 million individuals infected with chronic HCV in the United States, it is projected that 1.47 million will develop liver cirrhosis, 350,000 will develop liver cancer, and almost 900,000 will die from HCV-related complications. ${ }^{2,5} \mathrm{HCV}$ infected patients consume a large proportion of healthcare resources in the United States. Between 2001 and 2010, HCV infected individuals accounted for almost 3 million outpatient, inpatient, and emergency department visits in the United States. ${ }^{6}$ Estimated health care costs associated with the treatment and care of chronic HCV was $\$ 6.5$ billion in 2011 , and is expected to peak at $\$ 9.1$ billion in $2024 .^{3} \ln 2007$, mortality from HCV infection surpassed human immunodeficiency virus (HIV), and this trend is projected to continue..$^{2,4}$

\subsubsection{Emerging Epidemic}

The incidence of HCV in the United States declined from 2000-2003 and plateaued during 2006-2010 with 800-1000 new

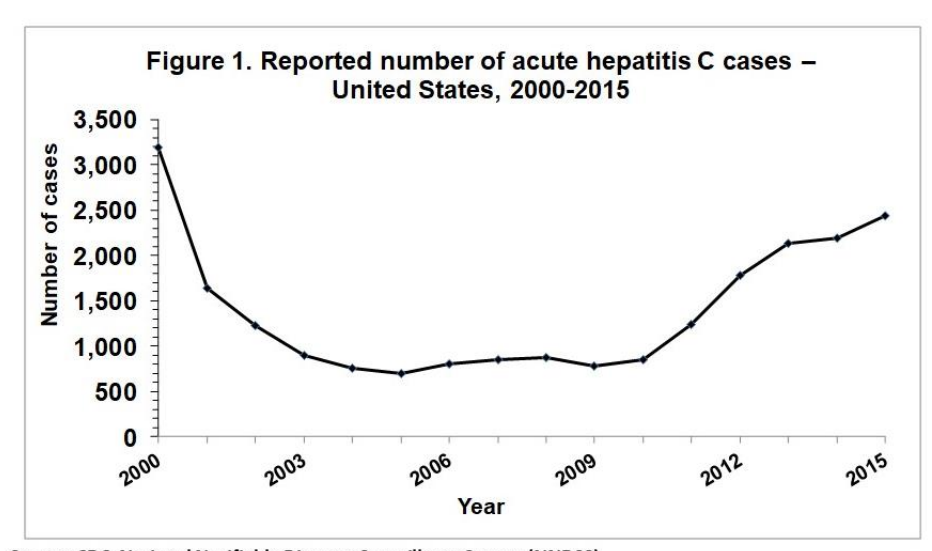

Source: CDC, National Notifiable Diseases Surveillance System (NNDSS) cases reported annually during this period. ${ }^{7}$ Thereafter, the number of reported acute 
cases significantly increased (Figure 1). ${ }^{7}$ Nationally, from 2010-2015 there was a 2.9 fold increase in the number of reported acute HCV infections. ${ }^{7}$

\subsubsection{The role of injection drug use and needle sharing}

Recent evidence examining the potential contributing factors underlying the $\mathrm{HCV}$ epidemic point to the prominent role of injection drug use..$^{2,7-12}$ People who inject drugs (PWID) account for $60 \%-70 \%$ of new HCV infections in the United States. ${ }^{2}$ The prevalence of HCV infection in PWIDs ranges between $40 \%$ and $90 \%$ and has been observed to be as high as $98 \% .^{13}$ Many of these PWIDs are white, ${ }^{2,9,11,12}$ young $(<35$ years of age), $, 2,-9,11,12$ and have a history of prescription drug use and abuse, especially prescription opiates. ${ }^{2,10-12}$ Among PWIDs, HCV is primarily transmitted through shared (used) injection syringes. ${ }^{14}$ A recent meta-analysis found a $94 \%$ increased risk of acquiring HCV infection among PWIDs who shared syringes (pooled risk ratio $(R R)=$ $1.94,95 \%$ confidence interval $(\mathrm{Cl}), 1.53,2.46) .{ }^{14}$

\subsubsection{The role of rural injectors}

Two recent studies suggest HCV infected PWIDs are more likely to reside in nonurban areas. ${ }^{11,12}$ Between 2006 and 2012, there was a staggering 364\% increase in HCV cases observed in young ( $\leq 30$ years of age) PWIDs in Central Appalachia (Kentucky, Tennessee, Virginia, and West Virginia). ${ }^{8}$ A recent heroin epidemic in this region is thought to underlie the HCV epidemic. ${ }^{8,15}$

\subsection{Needle Exchange Programs}

Needle exchange programs (NEP) are one popular harm reduction intervention that provide clean needles in exchange for used needles to minimize the reuse of needles contaminated with pathogens (i.e., HCV) during drug injection. ${ }^{16}$ 


\subsubsection{Unclear Efficacy}

The evidence for the effectiveness of NEPs in preventing HCV among PWIDs is mixed. ${ }^{17}$ A review of reviews by MacArthur et al. ${ }^{17}$ concluded that there was sufficient evidence to support the effectiveness of NEPs in reducing risky injection behavior (e.g., needle sharing), but found insufficient evidence to support or discount the effectiveness of NEPs for the prevention of HCV in PWIDs. A systematic review with meta-analysis of several interventions, including NEPs, to prevent HCV infection in PWIDs observed an increased risk of HCV seroconversion associated with NEP use (RR $1.62,95 \% \mathrm{Cl}, 1.04,2.52)$, although substantial between-study heterogeneity was observed $\left(Q=32.3, \mathrm{P}<0.01, R^{2}=81 \%\right) .{ }^{13}$ Additionally, this systematic review only included studies published through April 2010 when the current HCV epidemic was relatively young. A more recent systematic review of needle/syringe programs for the reduction of HCV infection among PWIDs by Abdul-Quader et al. ${ }^{18}$ found that 6 of the 15 included studies (40\%) reported decreases in HCV infection. NEPs have also been shown to effectively reduce the number of injections per syringe. ${ }^{19}$

\subsubsection{Barriers to Using Clean Needles and NEP Efficacy}

Although the ability to access clean needles is one important step in fighting the HCV epidemic, there are potential barriers to practicing risk reduction strategies (e.g., not sharing syringes) that may reduce the efficacy of NEPs. Barriers to using a clean needle for every injection can occur at the individual (e.g., being in drug withdraw), interpersonal (e.g., peer influences), and societal (e.g., confiscation of needles by police) levels of influence. For example, Phillips et al. ${ }^{19}$ recruited heroin injectors in Denver, Colorado and administered a structured interview that explored barriers 
associated with risk reduction practices. Being in drug withdrawal and fear of being arrested with needles were the two most frequently reported barriers to using new (clean) needles in this sample. Furthermore, secondary analysis of data from a randomized controlled trial that was designed to reduce the risk of human immunodeficiency virus (HIV) and sexually transmitted infections among female PWID sex workers observed that safe injection self-efficacy was negatively affected by police syringe confiscation. ${ }^{20}$ Similarly, Pollini et al. ${ }^{21}$ conducted a cross-sectional survey of PWIDs in Tijuana and Ciudad Juarez, Mexico to elucidate the association between syringe possession arrests and syringe sharing. After adjustment, arrests for possessing both clean needles (aOR $2.05,95 \% \mathrm{Cl}, 1.26,3.35)$ and used needles (aOR $2.87,95 \% \mathrm{Cl}, 1.76,4.69)$ were independently associated with receptive syringe sharing.

\subsection{Knowledge Gaps and Dissertation Aims}

Although previous studies have demonstrated the important role of NEPs in preventing HCV infection among PWIDs, none were conducted in rural areas of the United States. These rural areas, particularly Central Appalachia, are in the midst of a heroin epidemic that is directly contributing to the ongoing, costly HCV epidemic. However, the most recently available systematic reviews of the evidence do not include any studies that were conducted in rural areas of the United States. Therefore, there is a critical need to improve this evidence by conducting an updated systematic review of the literature with meta-analytic methods. Additionally, it is necessary to identify programmatic challenges to implementing these programs in rural areas by interviewing NEP directors and stakeholders. Furthermore, the presence of NEPs in rural communities may not be enough to prevent HCV transmission among PWIDs. 
Individual, interpersonal, and societal barriers to the use of clean needles may reduce the efficacy of NEPs in preventing HCV infection. However, no studies describing these barriers have been conducted in rural areas of the United States. Therefore, there is a critical need to understand the unique context and programmatic challenges of rural NEPs to design and implement successful programs to reduce HCV transmission in this underserved population. This dissertation proposes to address these knowledge gaps via the following three studies:

- Specific Aim \#1: Conduct a systematic literature review with metaanalysis on the efficacy of NEPs in preventing HCV infection among PWIDs.

Hypothesis: NEPs will demonstrate a protective effect on the acquisition of HCV infection among PWIDs.

- Specific Aim \#2: Identify and compare programmatic facilitators, barriers, and innovative solutions between two rural NEPs.

Research Questions: What are the facilitators and barriers to opening the program? What are the facilitators and barriers encountered during the day-today running of the program? What are the future plans for the program, and what are challenges and barriers related to these future plans?

- Specific Aim \#3: Identify self-reported barriers to using a clean needle among PWIDs attending NEPs in West Virginia.

Hypothesis: Fear of arrest and drug withdrawal will be the most frequently reported barriers to using a clean needle. 
Successful completion of these aims will result in an updated understanding of the efficacy of NEPs for preventing HCV, and provide previously unknown information concerning the operation of these programs in rural areas. This new information may inform the design, implementation, and operation of NEPs in other rural areas to mitigate the current HCV epidemic in this underserved population.

\section{APPROACH}

\subsection{Specific Aim \#1}

Conduct a systematic literature review with meta-analysis on the efficacy of NEPs in preventing HCV infection among PWIDs.

Hypothesis \#1: NEPs will demonstrate a protective effect on the acquisition of HCV infection among PWIDs.

\subsubsection{Study Design}

The conduct and reporting of this systematic review with meta-analysis will follow the recommended guidelines from the Preferred Reporting Items for Systematic Reviews and Meta-Analysis (PRISMA) statement. ${ }^{22}$ This review is registered in the PROSPERO trial registry (CRD42016035315).

\subsubsection{Study Eligibility}

The a priori inclusion criteria are as follows: (1) observational studies, (2) PWIDs, (3) NEP use, (4) HCV status ascertained by serological testing (saliva or serum), (5) studies published in any language after January 1,1989, and (6) data available or calculable for measures of association between participation in a NEP and HCV infection. Studies will be excluded based on inappropriate study design (i.e., 
randomized trials), population, intervention (i.e., supervised injection facility, pharmacybased NEP), or lack of available information to calculate a measure of association between program participation and HCV infection.

\subsubsection{Data Sources}

The following databases will be searched: (1) PubMed, (2) Scopus, (3) Web of Science, and (4) CINAHL. Based on the recommendations of van Driel et al.., ${ }^{23}$ and current practice, ${ }^{23,24}$ no searches for unpublished works or reports will be conducted. The search strategy and terms were based on the work of the HCV synthesis project, ${ }^{25}$ and Abdul-Quader et al. ${ }^{18}$ The term "people who inject drugs" was included in the search since it is now commonly used to refer to injection drug users (Figure 2). Crossreferencing from retrieved studies and reviews will also be conducted. The number needed to read (NNR) will be calculated by taking the inverse of the precision, which is defined as the number of included studies divided by the total number of studies screened after removal of duplicates. ${ }^{26}$ All studies identified during the search will be stored in Endnote ${ }^{\circledR}$ version 7.4. ${ }^{27}$

\subsubsection{Study Selection}

Two researchers will

independently review, select, and

Figure 2. Example Search String
(hepatitis C OR HCV) AND (intravenous drug abuse OR intravenous
drug use OR drug misuse OR drug addict OR injecting drug use OR
drug abuse OR people who inject drugs OR PWID OR PWID) AND
(prevention OR risk factor OR epidemiology OR prevalence OR
incidence OR seroprevalence OR seroincidence OR seroconversion
OR genotype OR coinfect*) AND (needle exchange OR needle
exchange program OR syringe exchange program OR syringe
access program) AND ("1989/01/01"[Date - Publication] :
"3000"[Date - Publication]).

abstract data from eligible studies with discrepancies resolved by a third researcher, if needed. The risk of bias in selected studies will be assessed using the Newcastle- 
Ottawa Quality Assessment Scale (NOS). ${ }^{28}$ Consistent with previous research, no study will be excluded based on the risk of bias assessment. ${ }^{29}$

\subsubsection{Statistical Analysis}

The a priori plan is to conduct an aggregate data meta-analysis with the study as the unit of analysis. The primary outcome for this study is the association between HCV seroconversion (dependent variable) and participation in a NEP (independent variable) observed in either cohort studies that follow seronegative individuals over time or case-control studies. This outcome will be calculated as the log odds ratio (OR) or the log hazard ratio $(\mathrm{HR})$. Because HRs include a time component, ORs and HRs will be analyzed separately, as recommended. ${ }^{30}$ If reported, adjusted effects will be used as the primary outcome under the assumption that such effects have been adjusted for potential bias. Effect size estimates from individual studies will be pooled using a random effects model, which incorporates between study variance into the overall estimate. ${ }^{31}$ Between study heterogeneity will be evaluated using the $Q$ statistic, and the percentage of variation in effect estimates due to heterogeneity will be assessed using the $R^{2}$ statistic. ${ }^{32}$ Effect sizes will be calculated after each study is removed from the model in order to assess the influence of each study on the overall results. Cumulative meta-analysis, ranked by year of publication, will be conducted to examine the accrued results over time. Ninety-five percent confidence intervals that do not cross ' 1 ' will be considered statistically significant with values below 1 indicative of a decrease in the odds or risk of HCV seroconversion (evidence of a protective or positive effect). Values significantly above ' 1 ' will be considered to indicate a harmful or negative effect. Values that cross ' 1 ' will be considered to indicate no effect from NEP 
participation on the prevention of HCV infection. All analyses will be carried out using Comprehensive Meta-Analysis $\AA$ (version 3.0). ${ }^{33}$

\subsection{Specific Aim \#2}

Conduct a qualitative case study of two rural NEPs to identify programmatic facilitators, barriers, and innovative solutions.

Research Questions: What are the facilitators and barriers to opening the program? What are the facilitators and barriers encountered during the day-today running of the program? What are the future plans for the program, and what are challenges and barriers related to these future plans?

\subsubsection{Study Design and Sampling}

A multiple, intrinsic case study approach has been selected for the following reasons: 1) each case (NEP) is of interest in and of itself (intrinsic), ${ }^{34}$ 2) the goal of this study is to obtain an in-depth understanding of each case, ${ }^{35}$ and 3 ) each program (case) is a bounded system. ${ }^{35}$ Each case is located in West Virginia and was selected using a purposive sampling strategy. ${ }^{36}$ More specifically, a NEP operated within a free healthcare clinic and a NEP operated within a health department were purposefully selected to document variations between these two program structures.

\subsubsection{Qualitative Interviews}

In-depth interviews with each NEP director will be conducted. Other NEP staff and individuals within the community that are recommended by the NEP director will also be interviewed. Prior to conducting the interview, a structured interview guide will be developed. This guide will focus on the facilitators and barriers encountered during the past and present, as well as ones anticipated to occur in the future. The specific 
questions included in the interview guide will be informed by initial discussions with each director, and include questions of interest to him or her. Finally, a case study of the first NEP in the United States, Point Defiance, ${ }^{37}$ will also serve as a source of potential questions. Due to the interest in obtaining an in-depth understanding of programmatic facilitators and barriers from the viewpoint of the participants, each interview will be semi-structured to allow conversations to emerge and flow in naturalistic directions that may depart from the interview guide. ${ }^{38}$ Each interview will be recorded using a TasCAM DR-05® stereo handheld microSD card recorder with built-in omnidirectional microphones. ${ }^{39}$ At various points throughout each interview, the interviewer will summarize the discussion and seek clarification from the interviewee regarding the accuracy (validity) of the measurement. As recommended, policy and procedure manuals and websites will also be analyzed to triangulate emerging themes. ${ }^{40}$

\subsubsection{Data Analysis}

Each interview will be professionally transcribed verbatim and entered into NVIVO® 11 Pro software for analysis. ${ }^{41}$ Prior to formal analysis, each transcript will be read several times independently by two researchers with notes made regarding initial codes. Next, line by line coding will be conducted with the objective of identifying emergent categories and themes. The initial codebook will have a priori codes for past, present, and future, in which information on barriers and facilitators corresponding to each time period will be categorized. In the constructivist tradition, ${ }^{38}$ codes will be allowed to emerge from the data prior to categorization into themes to promote discovery of unexpected information. Cross case synthesis will be conducted to denote 
similarities, differences, and emerging themes both within and between the two cases. ${ }^{40}$ After initial coding and classification, intensive group discussion with a second researcher with a goal of simple consensus will be employed to finalize the codes and overall themes..$^{42-44}$ If needed, discrepancies will be resolved by a third party. This approach is recommended by several methodologists in order to preserve the interpretive process at the core of qualitative analysis, ${ }^{45}$ and is consistent with current case study practice, ${ }^{46}$ in specific, and qualitative research reporting criteria, ${ }^{47}$ in general. Naturalistic generalizations will be made by comparing emergent themes with previously published literature. ${ }^{35}$

\subsection{Specific Aim \#3}

Use a mixed-methods approach to identify self-reported barriers to using a clean needle among PWIDs attending NEPs in West Virginia.

Hypothesis: Fear of arrest and drug withdrawal will be the most frequently reported barriers to using a clean needle.

\subsubsection{Study Design}

We propose to use a mixed methods approach to explore barriers to using a clean needle as reported by attendees of the first two NEPs to operate in West Virginia.

\subsubsection{Qualitative interviews}

There are no known validated surveys to assess barriers to using a clean needle among PWIDs. Therefore, we propose to modify the Barriers to Using New Needles ${ }^{19}$ survey (Figure 3) and create a standardized survey to ensure that all respondents answer the same set of questions (i.e., reliability). ${ }^{48}$ To further enhance survey reliability and increase validity, these questions will be reviewed by the directors of both NEPs 
from which attendees will be sampled. Modifications will be made to survey questions to maximize their applicability to the rural setting and avoid poorly defined words or terms that are not universally understood. ${ }^{48}$ Next, 3 to 5 attendees at each NEP will be purposively selected and interviewed using methods similar to the technique of respondent debriefings. ${ }^{49}$

Specifically, each attendee will be queried about survey comprehension and completeness. ${ }^{50}$ These attendees will also be asked

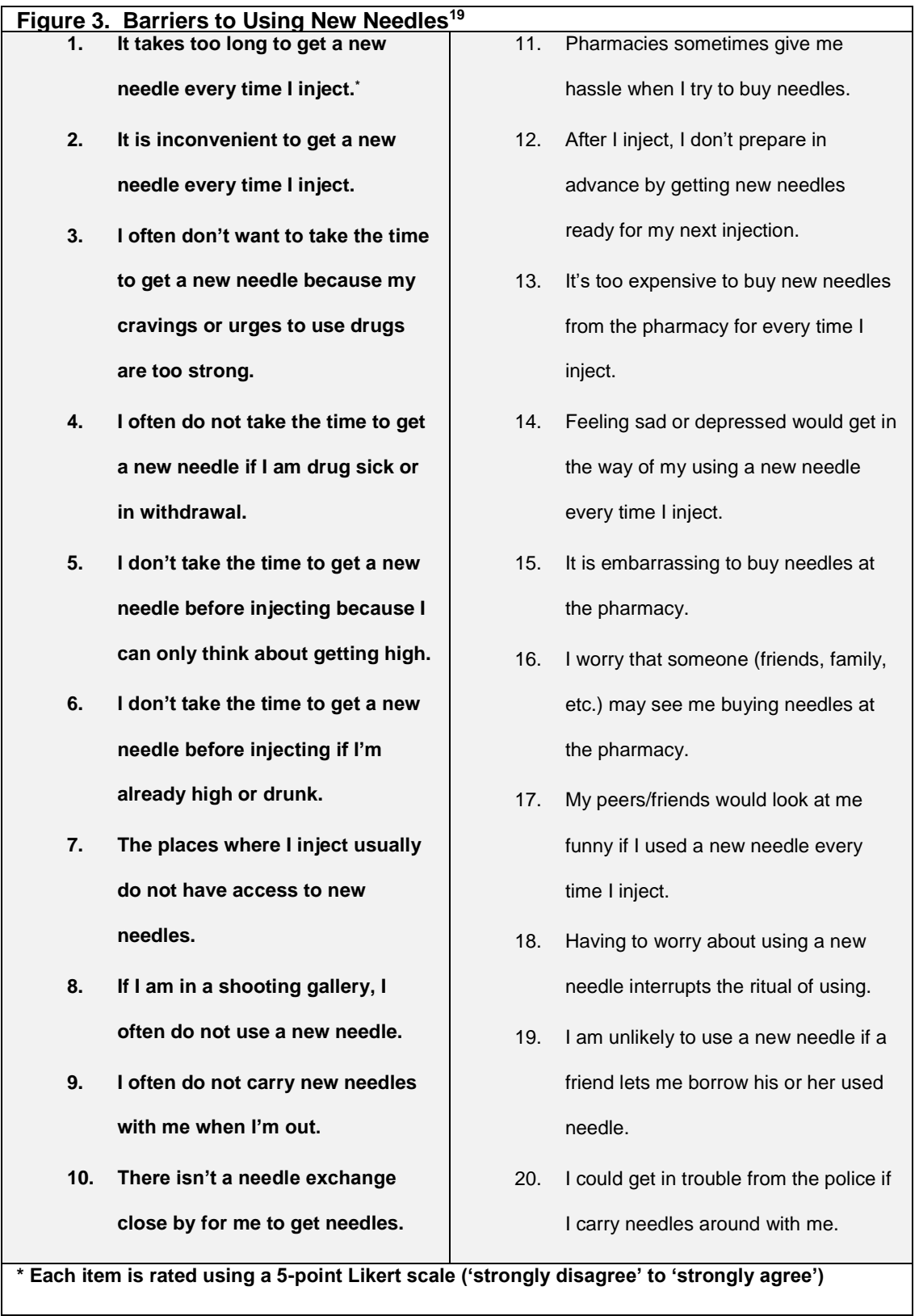

questions constructed from the emergent themes identified in Aim \#2 to achieve triangulation and thematic saturation. Final modifications based on these attendee interviews will be made in preparation for survey administration.

Basic demographic questions, along with questions querying injection history (e.g., frequency of injecting, years of injecting, etc.) will be included in the survey. To 
minimize potential bias stemming from participant and item nonresponse, the final survey will be designed and pilot tested to take no more than a few minutes to complete. ${ }^{50,51}$ Importantly, to minimize social desirability bias given the sensitive nature of the questions, the survey will be anonymous. ${ }^{48}$ Given the unstable and transient nature of the target population, and based on consultation with the NEP program directors, a convenience sample of attendees conducted over a few, consecutive exchange days is proposed. To further promote the number of responses, participants will be personally invited by study staff to take the survey while attending the exchange..$^{51}$

\subsubsection{Quantitative analysis}

All survey data will be entered into $\mathrm{JMP} \AA 13.0$ Pro. ${ }^{52}$ Frequencies and descriptive statistics will be calculated. Contingency table analysis will be used to explore associations between various demographic and injection history factors and individual barriers to clean needle use. An alpha of 0.05 has been selected as the threshold for statistical significance. 


\section{MANUSCRIPTS}

\subsection{Study 1 Manuscript}

Davis SM, Daily S, Kristjansson AL, Kelley GA, Zullig K, Baus A, Davidov D, Fisher M. Needle exchange programs for the prevention of hepatitis $C$ virus infection in people who inject drugs: a systematic review with meta-analysis. Harm Reduct J. 2017 May 17;14(1):25. doi: 10.1186/s12954-017-0156-Z. PubMed PMID: 28514954; PubMed Central PMCID: PMC5436422. 


\title{
Needle exchange programs for the prevention of hepatitis $C$ virus infection in people who inject drugs: a systematic review with meta-analysis
}

Stephen M. Davis ${ }^{1^{*}}$ D, Shay Daily², Alfgeir L. Kristjansson², George A. Kelley ${ }^{3}$, Keith Zullig², Adam Baus², Danielle Davidov ${ }^{1,2}$ and Melanie Fisher ${ }^{4}$

\begin{abstract}
Background: Previous research on the effectiveness of needle exchange programs (NEP) in preventing hepatitis $C$ virus (HCV) in people who inject drugs (PWID) has shown mixed findings. The purpose of this study was to use the meta-analytic approach to examine the association between NEP use and HCV prevention in PWIDs.

Methods: Study inclusion criteria were (1) observational studies, (2) PWIDs, (3) NEP use, (4) HCV status ascertained by serological testing, (5) studies published in any language since January 1, 1989, and (6) data available for measures of association. Studies were located by searching four electronic databases and cross-referencing. Study quality was assessed using the Newcastle Ottawa (NOS) scale. A ratio measure of association was calculated for each result from cohort or case-control studies and pooled using a random effects model. Odds ratio (OR) and hazard ratio (HR) models were analyzed separately. Results were considered statistically significant if the $95 \%$ confidence interval (Cl) did not cross 1 . Heterogeneity was estimated using $Q$ and $P^{2}$ with alpha values for $Q \leq 0.10$ considered statistically significant.

Results: Of the 555 citations reviewed, 6 studies containing 2437 participants were included. Studies had an average NOS score of 7 out of $9(77.8 \%)$ stars. Concerns over participant representativeness, unclear adjustments for confounders, and bias from participant nonresponse and loss to follow-up were noted. Results were mixed with the odds ratio model indicating no consistent association $(\mathrm{OR}, 0.51,95 \% \mathrm{Cl}, 0.05-5.15)$, and the hazard ratio model indicating a harmful effect $(\mathrm{HR}, 2.05,95 \% \mathrm{Cl}, 1.39-3.03)$. Substantial heterogeneity $(p \leq 0.10)$ and moderate to large inconsistency $\left(P^{2} \geq 66 \%\right)$ were observed for both models.
\end{abstract}

Conclusions: The impact of NEPs on HCV prevention in PWIDs remains unclear. There is a need for well-designed research studies employing standardized criteria and measurements to clarify this issue.

Trial registration: PROSPERO CRD42016035315

Keywords: Needle exchange program, Meta-analysis, Systematic review, Hepatitis C, Injection drug use, Opioids, Heroin, Pain killers, Pain

\footnotetext{
* Correspondence: smdavis@hsc.wvu.edu

${ }^{1}$ School of Medicine, Department of Emergency Medicine, Robert C. Byrd Health Sciences Center, West Virginia University, PO Box 9149, Morgantown, WV 26506-9149, USA

Full list of author information is available at the end of the article
} 


\section{Background Rationale}

Globally, over 184 million people ( $>2.8 \%$ of the world's population) have been infected with the hepatitis $C$ virus $(\mathrm{HCV})[1] . \mathrm{HCV}$ is a blood-borne virus that infects the liver. Approximately $75 \%$ of acute HCV infections become chronic [2]. Chronic HCV infection significantly increases the risk of liver disease, especially cirrhosis and liver cancer [1-7]. A majority of the 350,000 deaths attributed to HCV infection each year are caused by cirrhosis and hepatocellular carcinoma [7]. In most countries, the annual incidence of $\mathrm{HCV}$ infection has peaked with the exception of Russia where new cases are still increasing [1]. However, a troublesome pattern of new HCV cases observed in the USA suggests another or recurrent public health epidemic $[1,2,8,9]$.

The incidence of $\mathrm{HCV}$ in the USA declined from 2000-2005 and plateaued during 2005-2010 [8]. Thereafter, the number of reported acute cases increased significantly with a 2.6-fold increase observed between 2010 and 2014 [8]. Collectively, a 364\% increase in HCV cases among persons aged $\leq 30$ years was observed in Central Appalachia (Kentucky, Tennessee, Virginia, and West Virginia) between 2006 and 2012 [10]. As a result, the USA has set a goal of reducing new hepatitis $C$ infections from 0.28 cases per 100,000 to 0.25 cases per 100,000 (Healthy People 2020 Objective IID-26) [11].

$\mathrm{HCV}$-infected patients consume a large proportion of healthcare resources in the USA. Between 2001 and 2010, $\mathrm{HCV}$-infected individuals accounted for almost 3 million outpatient, inpatient, and emergency department visits in the USA [6]. Estimated healthcare costs associated with the treatment and care of chronic HCV was $\$ 6.5$ billion in 2011 and is expected to peak at $\$ 9.1$ billion in 2024 [3].

Although HCV can be transmitted in several ways, the primary mode of $\mathrm{HCV}$ exposure is percutaneous with injection drug use remaining the largest risk factor for HCV infection [2, 3, 8, 9, 12-18]. People who inject drugs (PWID) account for approximately $60-70 \%$ of the incidence of new HCV infections in the USA and many other countries [2]. Globally, it is estimated there are 10 million PWIDs that have HCV infection [18]. The prevalence of $\mathrm{HCV}$ infection in PWIDs ranges between 40 and $90 \%$ and has been observed to be as high as $98 \%$ [10]. Recent evidence from the USA has shown that many of these PWIDs are White [2, 9, 14, 15] and young $(<35)[2,8,9,14,15]$ and have a history of prescription drug use and abuse, especially prescription opiates [2, 13-15]. Furthermore, two recent studies suggest that HCVinfected PWIDs are more likely to reside in non-urban areas $[14,15]$.

Harm reduction interventions aim to reduce individual and societal harms stemming from drug use by targeting risky behaviors and risky settings [19]. A needle exchange program (NEP) is one popular harm reduction intervention that seeks to reduce risky settings. NEPs provide clean needles in exchange for used needles to minimize the reuse of needles contaminated with infectious disease during drug injection [19]. Many NEPs also provide other prevention materials and services such as additional sterile injecting supplies (e.g., cotton and alcohol swabs), wound care and safe injecting practices education, and linkage and referral to substance treatment programs for those PWIDs ready to quit injecting [20]. However, the evidence for the effectiveness of NEPs in preventing HCV among PWIDs is mixed [21]. For example, a systematic review with meta-analysis of several interventions, including NEPs, to prevent HCV infection in PWIDs observed an increased risk of $\mathrm{HCV}$ seroconversion associated with NEP use (RR 1.62, 95\% CI, 1.04-2.52), although substantial heterogeneity was observed $(Q=32.3 ; P<0.01$; $I^{2}=81 \%$ ) [12]. Furthermore, this systematic review only included studies published through April 2010, and selected studies were limited to the injection of illegal drugs (heroin, amphetamine, and cocaine) by PWIDs. Thus, studies describing the injection of prescription opioids were not considered for inclusion in the analysis. In another study, a review of reviews by MacArthur et al. [21] concluded that there was insufficient evidence to support or discount the effectiveness of NEPs for the prevention of HCV in PWIDs. A more recent systematic review of needle/syringe programs for the reduction of $\mathrm{HCV}$ infection among PWIDs by Abdul-Quader et al. [22] found that 6 of the 15 included studies (40\%) reported decreases in $\mathrm{HCV}$ infection. However, included studies only examined structural and population level interventions, as opposed to the association between individual use of NEPs and HCV infection. Finally, a recently published systematic review with meta-analysis by Sawangiit et al. [23] examined the effectiveness of pharmacy-based NEPs for PWIDs. However, in addition to specifically focusing on pharmacy-based NEPs, which may not always exchange needles [24], this study only examined the impact of these programs on the prevalence of HCV versus the prevention of new infections (incidence). To the best of the authors' knowledge, no other systematic reviews with or without meta-analyses of the effectiveness of NEPs in preventing $\mathrm{HCV}$ in PWIDs currently exist. Therefore, the primary objective of this study was to conduct a systematic review with meta-analysis to examine the association between NEPs and the prevention of HCV in PWIDs.

\section{Methods}

\section{General procedure}

The conduct and reporting of this study followed the recommended guidelines from the Preferred Reporting Items for Systematic Reviews and Meta-Analysis (PRISMA) statement [25]. This systematic review with 
meta-analysis is registered in the PROSPERO trial registry (CRD42016035315).

\section{Study eligibility criteria}

The a priori inclusion criteria for this systematic review with meta-analysis were as follows: (1) observational studies, (2) PWIDs, (3) NEP use, (4) HCV status ascertained by serological testing (saliva or serum), (5) studies published in any language after January 1, 1989, and (6) data available or calculable for measures of association between participation in a NEP and HCV infection. Studies were excluded based on inappropriate study design, population, intervention, or lack of available information to calculate a measure of association between program participation and HCV infection.

Although randomized controlled trials are considered the highest level of evidence for examining the effect of an intervention on a health outcome, [26] it was anticipated that no such trials would be found given that it would be highly unethical (violation of the ethical principle of beneficence) to randomize subjects away from a potentially beneficial treatment (i.e., needle exchange). Therefore, the focus of the review was on observational studies.

An a priori decision was made to exclude studies that reported the use of supervised injection facilities (SIFs). The rationale for this exclusion was based on the observation that while SIFs provide a safe environment for drug users to inject drugs, such facilities may not always provide (exchange) clean needles [27]. Additionally, studies describing the distribution of clean syringes from pharmacies were also excluded because such programs typically involve the sale of clean syringes with or without a prescription but may not involve the exchange of clean needles for dirty needles [24]. HCV status ascertained by serological testing was chosen as the primary outcome because previous research has demonstrated low sensitivity when HCV status is self-reported by PWIDs [28]. The year 1989 was chosen as a starting point for the search because this was the year that the HCV antibody was identified, and thus, enabled serological testing to detect the virus [29]. Based on the recommendations by van Driel et al. [30], no searches for unpublished works such as dissertations and conference abstracts or other unpublished reports were conducted.

\section{Data sources}

The following databases were searched between July 18 , 2016, and August 24, 2016: (1) PubMed, (2) Scopus, (3) Web of Science, and (4) CINAHL. The search strategy and terms were based on the work of the HCV synthesis project [29] and was modified to include specific search terms related to NEP that were used by Abdul-Quader et al. [22] in their systematic review of population level outcomes following the implementation of NEP. Additionally, the term "people who inject drugs" was included in the search given the observation by the authors that this term has been commonly used to refer to injection drug users in more recent research literature. Although there was slight variation in the specific search format between databases, the following search terms and combinations were used: (hepatitis C OR HCV) AND (intravenous drug abuse OR intravenous drug use OR drug misuse OR drug addict OR injecting drug use OR drug abuse OR people who inject drugs OR PWID OR PWID) AND (prevention OR risk factor OR epidemiology OR prevalence OR incidence OR seroprevalence OR seroincidence OR seroconversion OR genotype OR coinfect*) AND (needle exchange OR needle exchange program OR syringe exchange program OR syringe access program) AND ("1989/01/01" [Date - Publication] : "3000" [Date - Publication]). Search strategy examples for the four databases searched are included in Additional file 1. In addition to database searches, cross-referencing from retrieved studies and reviews was also conducted. After identifying the final number of studies to be included, the number needed to read (NNR) was calculated by taking the inverse of the precision, which was defined as the number of included studies divided by the total number of studies screened after removal of duplicates [31]. All studies identified during the search were stored in EndNote ${ }^{\bullet}$ version 7.4 [32].

\section{Study selection}

Two researchers (SMD and SD) independently reviewed studies for selection and abstracted data from eligible studies with discrepancies resolved by consensus and discussion with a third researcher (GK), if needed. Duplicate studies were discovered by using the "Find Duplicates" tool in EndNote ${ }^{\circ}$ 7.4 [32] and by manual examination. After removal of duplicate studies, abstracts of all studies were reviewed and the full text of studies appearing to meet the inclusion criteria were obtained and reviewed. Studies that met all of the inclusion criteria were selected. The authors were not blinded to journal titles and study authors and their associated institutions during the review. Reasons for exclusion from further review were coded as one or more of the following: (1) inappropriate population (i.e., not PWIDs), (2) inappropriate intervention (i.e., not a NEP), (3) inappropriate comparison (i.e., no comparison to non-exchange users), (4) inappropriate outcome (i.e., self-reported HCV status), and (5) lack of data to enable calculations of the association between program use and $\mathrm{HCV}$ infection.

\section{Data abstraction}

A codebook containing 85 items was developed a priori using Microsoft Excel 2013 ${ }^{\circ}$, [33] and is included in 
Additional file 2. The major categories of variables coded by the authors were based upon the HCV Synthesis Project [29] and included (1) study characteristics (author, journal, year, funding status, design, inclusion criteria, recruitment method, recruitment locations, method of determining PWID status, specimen type, and HCV test method), (2) participant characteristics (age, gender, ethnicity, duration of drug use, type of drug used, frequency of use), and (3) outcome characteristics (prevalence, incidence, number of person years, sample size, and variables adjusted for, if applicable). The primary outcome of this study, established a priori, was the association between HCV seroconversion and use of a NEP.

\section{Risk of bias assessment}

The risk of bias in selected studies was assessed using the Newcastle-Ottawa Quality Assessment Scale (NOS) [34]. Consistent with previous research, no study was excluded based on the risk of bias assessment [35].

\section{Statistical analysis}

The a priori plan was to conduct an aggregate data meta-analysis with the study as the unit of analysis.

\section{Calculation of effect sizes}

The primary outcome for this study was the association between $\mathrm{HCV}$ seroconversion and participation in a NEP observed in either cohort studies that follow seronegative individuals over time to monitor seroconversion or case-control studies. This outcome was calculated as the $\log$ odds ratio $(\mathrm{OR})$ or the log hazard ratio (HR). Because hazard ratios include a time component, ORs and HRs were analyzed separately.

Where possible, published ratios (OR or HR) and confidence limits from individual studies were used to calculate the $\log$ ratios and corresponding $\operatorname{logs}$ of the standard errors. If associations in individual studies were not presented in ratios, only log odds ratios were calculated using the reported number of $\mathrm{HCV}$ infections and the total number of participants in each group (NEP users and non-users). Missing log hazard ratios were not calculated due to the unavailability of time data. If an exact $p$ value was reported instead of a confidence interval $(\mathrm{CI})$, the standard error was calculated using the following formula [26]: $\log (\mathrm{OR}) / \mathrm{z}$. If reported, adjusted effects were used as the primary outcome under the assumption that such effects have been adjusted for potential bias in the observed association between NEP participation and HCV infection. For ease of interpretation, log ratios were converted back to odds ratios and hazard ratios after analysis.

\section{Pooling estimates}

Effect size estimates from individual studies were pooled using a random effects model [36]. Between-study heterogeneity was evaluated using the $Q$ statistic, and the percentage of variation in effect estimates due to heterogeneity was assessed using the $I^{2}$ statistic [37]. Based on current recommendations, heterogeneity was considered to be substantial if the $p$ value for the observed $Q$ statistic was $\leq 0.10$ [26]. The amount of heterogeneity present (as assessed by $I^{2}$ values) was interpreted according to the following categories: $<25 \%$ ("very low"); 25 to $<50 \%$ ("low"); 50 to $<75 \%$ ("moderate"); and $75 \%$ or greater ("large") [37]. Effect sizes were calculated after each study was removed from the model in order to assess the influence of each study on the overall results. In addition, cumulative meta-analysis, ranked by year of publication, was conducted to examine the accrued results over time. Ninety-five percent confidence intervals that did not cross 1 were considered to be statistically significant with values below one indicative of a decrease in the odds or risk of HCV seroconversion (evidence of a preventative or positive effect). Values significantly above one were considered to indicate a harmful or negative effect. Values that crossed 1 were considered to indicate no effect from NEP participation on the prevention of HCV infection.

An a priori plan was made to assess small-study effects (publication bias, etc.) using funnel plots and Egger's regression intercept test (one-tailed). However, we were unable to conduct these analyses because we did not have at least 10 effect sizes, the minimum sample size recommended by Sterne et al. [38]. Similarly, a priori plans to conduct a mixed-effects meta-regression to examine potential covariates and a moderator analysis to examine potential differential study effects from different study designs (e.g., cohort and case study) were not conducted due to insufficient sample sizes ( $<10$ effects) [26]. All analyses were carried out using Comprehensive Meta-Analysis (version 3.0) [39].

\section{Results \\ Study characteristics}

Overall, of the 555 references examined, 6 studies, [40-45] containing data from 2437 PWIDs, were included in the final review and analysis. One study [46] was identified that contained estimates based on data from the same sample of PWIDs collected in the same location during the same time periods as those included in another larger and more recent study that was selected for inclusion [42]. Therefore, this study was eliminated from analysis given that these data would have violated the statistical requirement of independence of effect size estimates. The precision of the search was $1 \%(6 / 555)$, and the NNR was 100 . Figure 1 diagrams the 


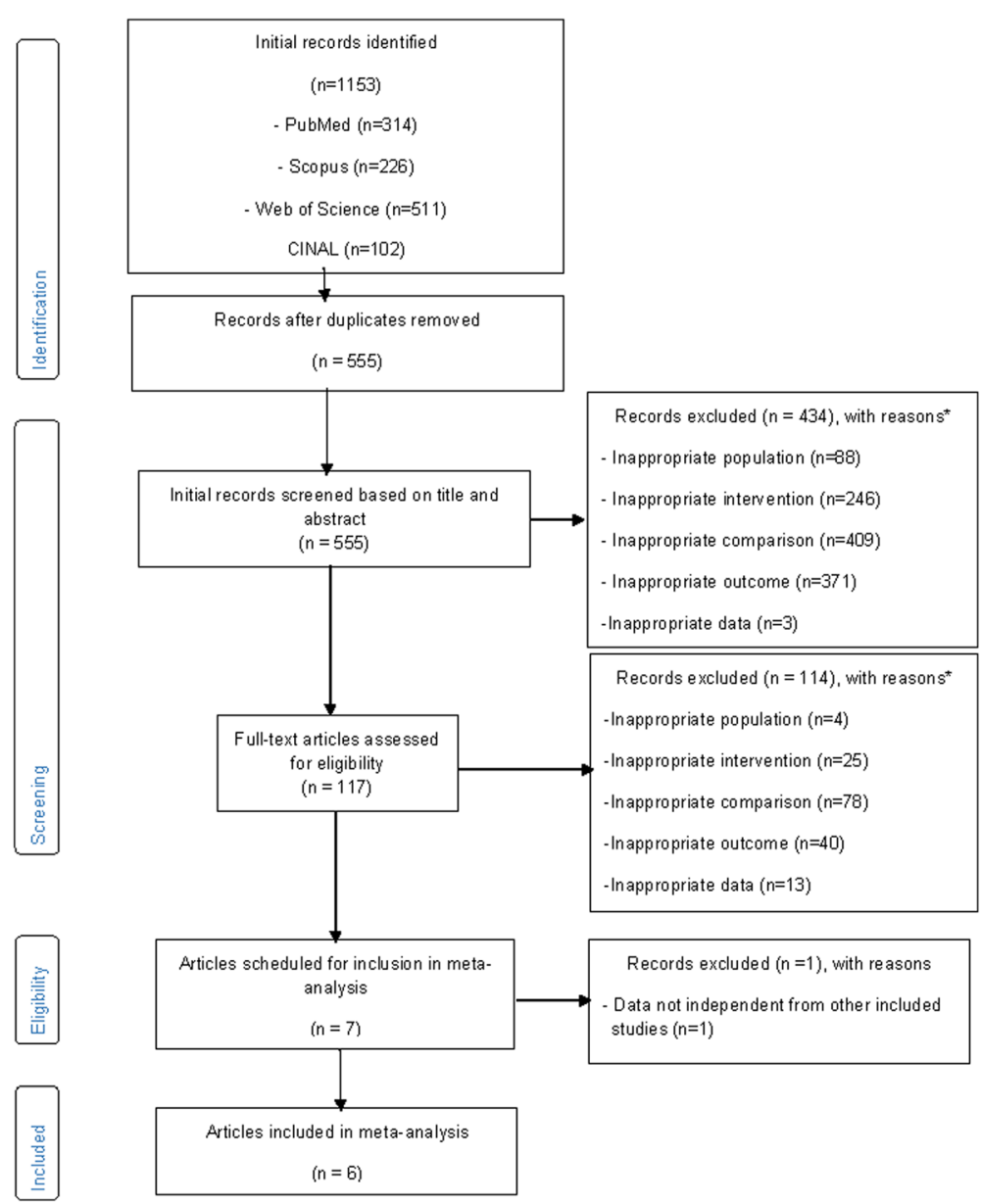

Fig. 1 Flow diagram for the selection of studies. *Number of reasons exceeded the number of studies because some studies were excluded for more than one reason

search process and includes reasons for the exclusion of various studies from the final analysis. Table 1 lists the general study characteristics. Half of the studies were conducted in the USA [40-42], followed by two conducted in Canada [43, 44], and one conducted in Afghanistan [45]. Without exception, all studies were conducted in densely populated urban locations. All studies were published in the English language. There was one case-control study [40] and five cohort studies [41-45].

\section{Participant characteristics}

All studies enrolled PWIDs who self-reported injections. However, time since last injection prior to enrollment varied between studies with two studies enrolling participants that had injected in the previous month [43, 45], one study enrolling those that injected in the previous 6 months [44], one study enrolling those that had injected in the past year [41], one study containing a mix of participants who injected in the previous 6 months and 1 year [42], and one study not specifying any length of prior injection [40].
Participants were enrolled in a variety of settings ranging from syringe exchange programs, [44] harm reduction programs which provided motivational counseling, washing facilities, medical care, and infectious diseases testing in addition to syringe distribution [45], emergency rooms [40], county health departments [40], jails [41], streets [41-43], social service agencies [41], and areas of known drug user congregation [45].

A variety of sampling schemes were described including respondent driven [42], criterion [40], convenience [44], random [41], and a variant of time-location sampling [45]. One study did not describe the sampling strategy [43]. Study participants were recruited over two decades with the oldest study recruiting during 1991 to 1993 [40] and the most recent study enrolling participants between June 2007 and March 2009 [45].

Of the 2437 PWIDs, 941 reported participation in a needle exchange program ("NEP users") and 946 participants did not report using a needle exchange ("non-users"). Two studies did not report needle exchange participation for the number of participants who were HCV seronegative at 


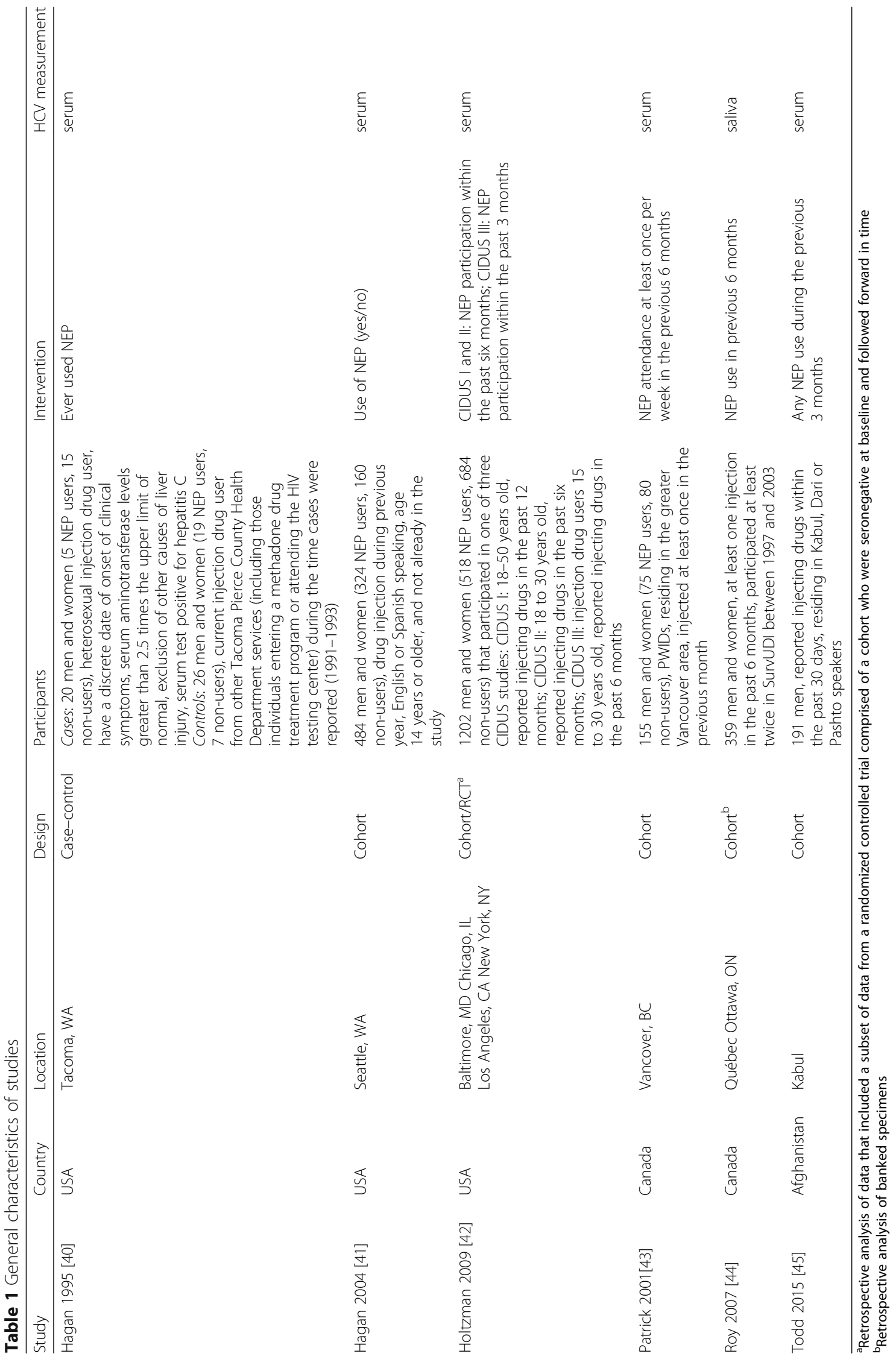


baseline [44, 45]. With the exception of one study that only enrolled males [45], all other studies enrolled both genders. The percentage of male participants in these studies ranged from a low of $42.3 \%$ [40] to a high of $73 \%$ [44] with an average of $62 \%$. Participants in all studies were generally young ( $<40$ years). However, reporting of age was variable with the average given in one study (31.8 years [44]), medians in two studies (28 years [43, 45]), and age categories in the remaining three studies [40-42]. In these latter three studies, the proportion of all participants less than 34 years of age was at least $65 \%$.

There was a wide variability in the reporting of participant race and/or ethnicity with two studies $[44,45]$ not reporting any race and/or ethnicity. Of the five studies reporting race and/or ethnicity, "White" race was the most frequently reported by participants, ranging from a low of $49 \%$ [42] to a high of $85 \%$ [40].

The types of drugs injected varied between studies. The most frequent drugs injected, self-reported by participants in each study, included heroin [41, 43] and cocaine [44]. Three studies [40, 42, 45] did not report a specific type of drug.

Length of time injecting was also widely variable between studies. Three studies reported median injecting durations of 2 years [42, 45] and 7 years [43]. Another two studies partitioned injecting duration into categories. Hagan et al. [40] reported the following categories: HCV positive cases: $<5$ years $(n=7,35 \%), 5+$ years $(n=13$, $65 \%)$, HCV negative controls: $<5$ years $(n=6,23.1 \%)$, $5+$ years $(n=20,76.9 \%)$. Hagan et al. [41] reported the following categories (years): $\leq 1 \quad(n=57,13 \%), 1.1$ to $2.0(n=76,16 \%), 2.1-5.0 \quad(n=144,32 \%), 5.1-10.0$ $(n=84,19 \%),>10.0(n=93,20 \%)$. Roy et al. [44] reported an average of 10 years of injecting.

Half of the studies did not report any information related to the frequency of injections [40, 44, 45]. In the three studies reporting injection frequency [41-43], the proportion of participants injecting at least once a day averaged 51.03\%.

\section{Intervention characteristics}

Participation in a NEP was assessed by self-report in all six studies. The frequency of participation varied between studies due to heterogeneity in the presentation of results. Two studies reported ever (versus never) using a NEP $[40,41]$. Two studies reported NEP use in the last 3 months $[42,45]$. Three studies reported NEP use in the last 6 months [42-44], with Patrick et al. [43] requiring NEP attendance at least once per week in the past 6 months.

\section{Risk of bias assessment}

Risk of bias assessment results are shown in Table 2. Individual study scores ranged from 5-8 stars, which
Table 2 New-Castle Ottawa Scale Ratings

\begin{tabular}{llll}
\hline Study & Selection & Comparability & Exposure/Outcome \\
\hline Hagan 1995 [40] & $\star \star \star$ & $\star \star$ & $\star$ \\
Hagan 2004 [41] & $\star \star \star \star$ & $\star \star$ & $\star \star$ \\
Holtzman 2009 [42] & $\star \star \star \star$ & $\star \star$ & $\star$ \\
Patrick 2001 [43] & $\star \star \star \star$ & $\star$ & $\star \star$ \\
Roy 2007 [44] & $\star \star \star$ & $\star$ & $\star$ \\
Todd 2015 [45] & $\star \star \star \star$ & $\star \star$ & $\star \star$
\end{tabular}

represented $55-89 \%$ of the total number of stars that can be awarded ( 9 stars). The five cohort design studies [41-45] averaged 7 stars with the lone case-control study [40] receiving 6 stars.

Four of the six studies [41-43, 45] received the maximum number of stars (4) in the selection category. Concerns over the representativeness of participants resulted in the deduction of one star from this category in the remaining two studies $[40,44]$. All but two of the studies received the maximum number of stars (2) in the comparability category. Patrick et al. [43] and Roy et al. [45] each received only one star in this category because adjustments for potential confounders were unclear. No studies received the maximum number of three stars in the assessment of the exposure (case-control) or outcome (cohort) category. Potential bias from participant nonresponse and loss to follow-up was a primary weakness for all studies.

\section{Primary outcome}

Study outcomes are shown in Table 3. Four studies reported (or had data enabling calculation of) hazard ratios [41, 43-45], with two studies reporting odds ratios $[40,42]$. Three studies $[40,42,43]$ adjusted effect estimates for potential confounders. Overall, findings were mixed. A statistically significant harmful effect from participation in NEPs was observed when the four studies that reported hazard ratios were combined (pooled HR, 2.05, 95\% CI, 1.39-3.03, Fig. 2a). However, significant heterogeneity and moderate inconsistency were observed $\left(Q=9.03 ; p=0.029 ; I^{2}=66.8 \%\right)$. This finding was not influenced by the deletion of any study from the model once (Fig. 2b) and remained consistent over time (Fig. 2c), with all cumulative results yielding confidence intervals that did not cross 1 . In contrast, there was no significant association between the odds of HCV seroconversion and participation in a NEP when the two studies that reported odds ratios were combined (pooled OR, 0.51, 95\% CI, 0.05-5.15, Fig. 3), although both significant heterogeneity and large inconsistency between studies were observed $\left(Q=8.66 ; p=0.003 ; I^{2}=88.4 \%\right)$. Influence analysis and cumulative meta-analysis were not conducted on the odds ratio model given the inclusion of only two studies. 
Table 3 Study outcomes and adjustments

\begin{tabular}{|c|c|c|c|c|c|c|c|}
\hline \multirow[b]{2}{*}{ Study } & \multirow[b]{2}{*}{ Outcome } & \multirow[b]{2}{*}{ Adjusted } & \multirow[b]{2}{*}{ Adjustments } & \multirow[b]{2}{*}{ Estimate } & \multicolumn{2}{|l|}{$95 \% \mathrm{Cl}$} & \multirow[b]{2}{*}{$p$} \\
\hline & & & & & Lower & Upper & \\
\hline Hagan 1995 [40] & Odds ratio & y & $\begin{array}{l}\text { Sex, race/ethnicity, duration of } \\
\text { drug injecting }\end{array}$ & 0.14 & 0.03 & 0.62 & \\
\hline Hagan 2004 [41] & Hazard ratio & $n$ & & 1.40 & 0.90 & 1.90 & \\
\hline Holtzman 2009 [42] & Odds ratio & y & $\begin{array}{l}\text { Sex, age in years, race/ethnicity, } \\
\text { education, source of income, site, } \\
\text { study time period, injection risk } \\
\text { behaviors, and HIV serostatus }\end{array}$ & 1.49 & 0.96 & 2.29 & \\
\hline Patrick 2001 [43] & Hazard ratio & y & Not described & 2.56 & 1.37 & 4.79 & \\
\hline Roy 2007 [44] & hazard ratio & $n$ & & 3.02 & & & 0.18 \\
\hline Todd 2015 [45] & Hazard ratio & $n$ & & 1.72 & 1.07 & 2.76 & \\
\hline
\end{tabular}

\section{Discussion}

The primary purpose of this systematic review with meta-analysis was to assess the potential effect of NEP on the prevention of $\mathrm{HCV}$ infection in PWIDs. The overall findings were mixed and suggest that NEP could either increase the risk of HCV infection in PWIDs or have no effect. This interpretation is supported by (1) pooled results from studies reporting a hazard ratio that indicate a harmful effect (pooled HR, 2.05, 95\% CI, 1.39-3.03), (2) pooled results from studies examining the odds of infection that do not indicate either a preventive benefit or harmful effect (pooled OR, 0.51, 95\% CI, $0.05-5.15)$, and (3) substantial heterogeneity $(p \leq 0.10)$ and moderate $\left(I^{2}=67 \%\right)$ to high $\left(I^{2}=88 \%\right)$ inconsistency observed for both models. These mixed findings are consistent with previous research.

A review of reviews without meta-analysis by MacArthur et al. [21] of interventions to prevent HCV in PWIDs identified 17 studies with mixed results ( 9 positive, 2 negative, and 6 no effect) leading to the conclusion that there was insufficient evidence to either support or discount the effectiveness of NEP for preventing HCV.

Hagan et al. [12] conducted a systematic review with meta-analysis of the effectiveness of interventions, including NEP, on the prevention of HCV. Similar to the results from our hazard ratio model, this meta-analysis observed a $62 \%$ increase in the risk of HCV seroconversion from participation in syringe access programs (RR, 1.62, 95\% CI, 1.04-2.52) with substantial heterogeneity and large inconsistency $\left(Q=32.3, I^{2}=81 \%\right)$. Included studies contained a mixture of no effect (three studies), positive (one study), and harmful (three studies) results. Five of these seven studies were included in our metaanalysis. A single-site study by Thorpe et al. [46] which observed no effect (HR, 1.29, 95\% CI, 0.6-2.79) from NEP participation on $\mathrm{HCV}$ infection contained data that were also included in the Holtzman et al. [42] multi-site study. Therefore, we excluded this study from the final model to maintain the criterion of independence of effect sizes. Despite extensive searching and multiple electronic and personal queries, we were unable to locate a governmental report by Lamonthe et al. [47] for review and possible inclusion in our systematic review with meta-analysis. Hagan et al. [12] reported that this study demonstrated a harmful effect (HR, 2.24, 95\% CI, 1.01-4.98). The current review included one additional cohort study [45], published in 2015, that observed a harmful effect.

A recently published systematic review with metaanalysis of pharmacy-based NEP demonstrated a 74\% reduction in the odds of $\mathrm{HCV}$ infection $(\mathrm{OR}=0.26,95 \%$ CI, 0.18, 0.38) associated with pharmacy-based NEP participation [23]. However, the authors cautioned that this finding was unclear due to the very small number of included studies $(n=2)$ and significant bias concerns. Observed heterogeneity in the study population, and variability in defining the intervention and outcomes reported, further precluded the ability to draw definitive conclusions between $\mathrm{HCV}$ infection and pharmacybased NEP participation. This observation is consistent with the current review. Indeed, the substantial heterogeneity and large inconsistency observed in both the current study and the previous meta-analyses may be related to important between-study differences in the population enrolled, intervention examined, outcome assessed, and type of study design (and associated statistical analyses).

\section{Variable populations}

All studies included in the current review, with the notable exception of Todd et al. [45], enrolled a mix of genders that were largely under the age of 40 and White. However, all studies had slightly different age requirements with some studies enrolling PWIDs as young as 14 years [41] and 15 years [43] compared to other studies that did not report a limit on age [40, 44], only enrolled adults ( $\geq 18$ years [45]), or had varying age range requirements (e.g., 18-30 and 18-40) [42]. Furthermore, 
a

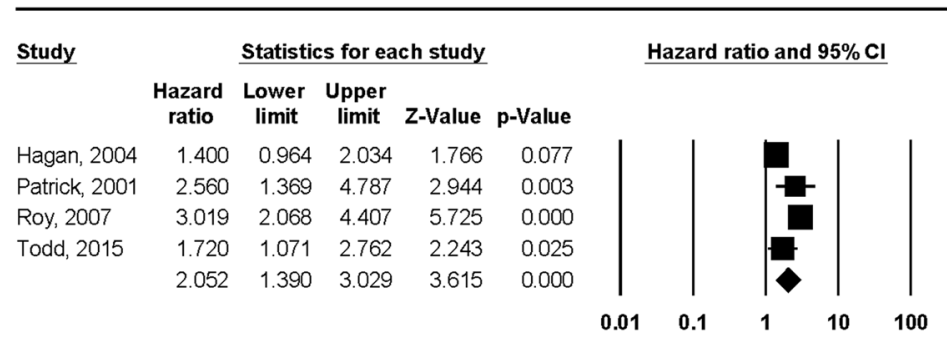

$\mathrm{Q}=9.03, \mathrm{p}=0.029 ; I^{2}=66.8 \%$
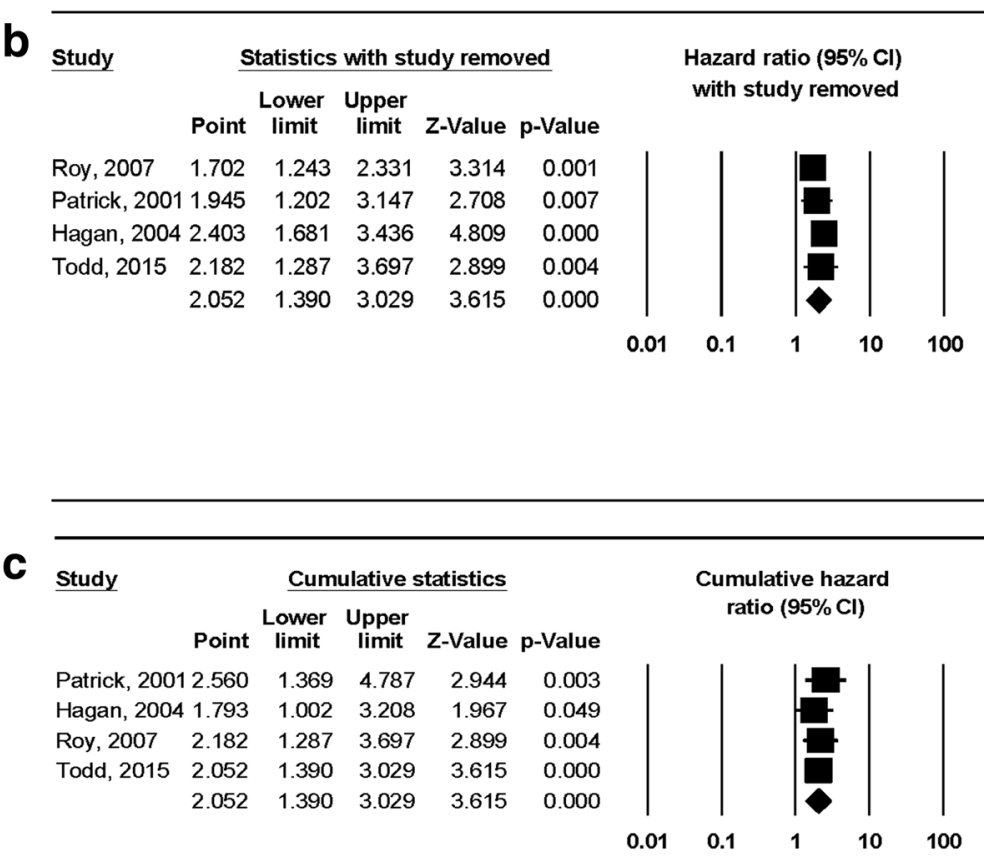

Fig. 2 a Forest plot for the risk of hepatitis $C$ infection among needle exchange program participants. The black squares represent the risk of hepatitis C infection observed in each study with the $95 \%$ confidence intervals represented by the lines on each side of the squares. The diamond represents the pooled risk of hepatitis C infection with the $95 \%$ confidence interval indicated by the left and right extremes of the diamond. $\mathbf{b}$ Influence analysis for the risk of hepatitis $C$ infection among needle exchange program participants. Influence analysis for point estimate changes in the risk of hepatitis $C$ infection with each individual study deleted from the model once. c Cumulative meta-analysis for the risk of hepatitis $C$ infection among needle exchange program participants. The results of each corresponding study, ordered by year of publication from oldest to newest, are pooled with all studies preceding it

there were a variety of settings in which PWIDs were recruited over an almost 20-year timeframe. However, with the exception of Todd et al. [45], all included studies were conducted in North America. Importantly, no studies took place in the rural, Central Appalachian region of the USA, an area that is in the midst of a hepatitis $\mathrm{C}$ epidemic that is directly related to increasing prescription opioid abuse and injection of heroin [48, 49]. Additionally, injection use appeared to be entirely selfreported in all studies, and there were variances in the length of time from last injection criterion between studies, with some studies enrolling individuals who reported ever injecting [40] to other studies only enrolling individuals who injected at least once in the previous month $[43,45]$.

\section{Variable interventions}

Participation in NEP was self-reported in all studies. However, the frequency of attendance was highly variable between studies, ranging from questions querying 


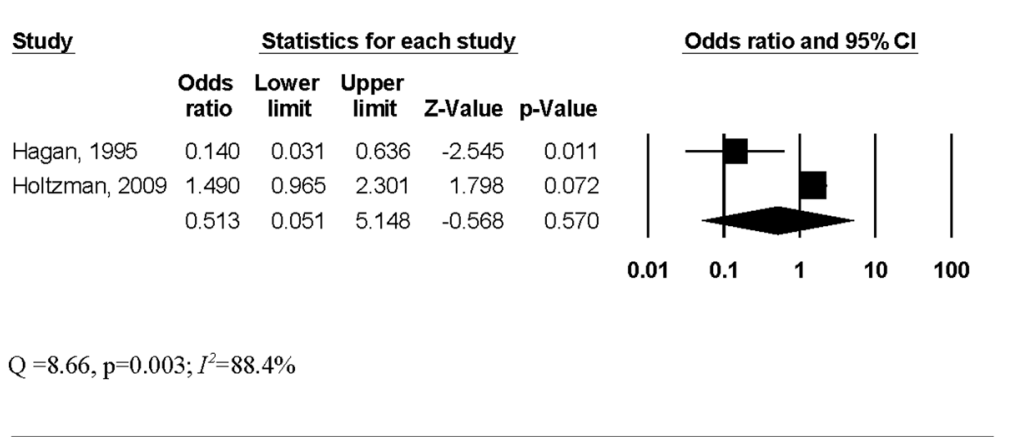

Fig. 3 Forest plot for the odds of hepatitis $\mathrm{C}$ infection among needle exchange program participants. The black squares represent the odds of hepatitis C infection observed in each study with the $95 \%$ confidence intervals represented by the lines on each side of the squares. The diamond represents the pooled odds of hepatitis C infection with the $95 \%$ confidence interval indicated by the left and right extremes of the diamond

whether or not a program had ever been used $[40,41]$ to NEP attendance at least once per week in the previous 6 months [43]. No pattern between study findings and reported frequency of attendance was observed.

\section{Variable outcomes}

Although all studies included a serological measure of $\mathrm{HCV}$, one study demonstrating a harmful effect used saliva measurements [44], which may vary in accuracy compared to serum tests. Additionally, the particular assay used in the serum measurements was variable with some studies using second-generation assays and others using third-generation assays.

\section{Variable study designs and analyses}

The particular type of outcome assessed was directly related to variability in study design and the associated outcomes. In the current review, cohort studies that incorporated a time component into the analysis and reported hazard ratios, demonstrated a significant increase in the risk of $\mathrm{HCV}$ infection for NEP users; whereas, the lone case-control study [40] that reported an odds ratio demonstrated a highly significant preventative effect from NEP use. In contrast, Holtzman et al. [42] analyzed data from a combination of observational cohort studies and a subset of data from a randomized behavioral intervention, and reported an odds ratio that demonstrated no significant effect from NEP participation on the prevention of HCV. These differences in outcomes precluded the ability to combine all effect sizes into one model and instead resulted in two models with different interpretations. Although this plan differs from the combined model presented by Hagan et al. [12], we made this decision a priori based on the rationale that odds ratios and hazard ratios are two fundamentally different measures, given that the latter incorporates a time component (i.e., time-to-event data). Although not recommended, time-to-event data can sometimes be analyzed as dichotomous data that yield odds ratios [26]. However, such an analysis requires that the status (e.g., serostatus) of all patients be known at a fixed time point (i.e., 12 months) [26], which was not reported in all studies included in our hazard ratio model. Further complicating matters is that some studies made adjustments to the overall reported main outcome whereas other studies only reported unadjusted results. Among those studies that made adjustments, different potential confounders were controlled for likely leading to additional heterogeneity between studies.

In a recent review of reviews, MacArthur et al. [21] reported a similar pattern of variability in findings by study design. Of the 17 studies examining the impact of NEP on the prevention of $\mathrm{HCV}$, nine studies (one case-control study, six cross-sectional studies, and two ecological studies) demonstrated a positive (or preventative) effect from NEP use. In contrast, two cohort studies demonstrated a negative (or harmful) effect. The remaining six studies demonstrated no association and were evenly split between three cohort studies and three cross-sectional studies.

While we made an a priori decision to exclude crosssectional studies in our meta-analysis due to the fact that such designs can only assess associations between NEP participation and $\mathrm{HCV}$ infection, the current review identified five cross-sectional studies that met all other inclusion criteria [50-54]. Results from these studies were mixed with one study finding no effect from NEP participation (OR, 1.54, 95\% CI, 0.73-3.24) [50], one study finding a preventative effect (OR, 0.59, 95\% CI, 0.43-0.77) [53], and three studies demonstrating a harmful effect (OR, 2.17, 95\% CI, 1.38-3.40 [51]; OR, 2.1, 95\% CI, 1.54-2.89 [52]; OR, 2.54, 95\% CI, 1.36-4.74 [54]). Similar to the findings from the current review, not all studies adjusted odds ratios for confounding, and the various adjustments made were not uniform between studies. Qualitative heterogeneity in the measurement of 
both the exposure (NEP use) and outcome (HCV infection) were also reported. Finally, Turner et al. [55] conducted a meta-analysis of cross-sectional and cohort studies conducted in the UK and observed no effect from NEP participation on $\mathrm{HCV}$ incidence (ES, 0.58, 95\% CI, 0.30-1.15). Interestingly, and in contrast to all other studies, no inconsistency $\left(I^{2}=0.0 \%\right)$ was reported in the model. The two cohort studies included in this model did not contain data that would allow calculation of the association between NEP use and HCV infection (inclusion criteria \#6).

\section{HIV studies}

The heterogeneity observed in this systematic review with meta-analysis is not limited to studies examining the effectiveness of NEP for the prevention of HCV. A recent systematic review with meta-analysis by Aspinall et al. [56] examining the influence of NEP on prevention of HIV observed large inconsistency $\left(I^{2}=75.7 \%\right)$ between the 12 included studies (10 cohort, 1 cross-sectional, and 1 case-control). Although a preventative effect from NEP exposure was suggested, the upper bound of the confidence interval slightly crossed 1 (pooled effect size, 0.66, $95 \%$ CI $0.42-1.01)$. When higher-quality studies, as graded by the Newcastle-Ottawa Scale, were combined, a significant preventative effect was observed $(0.42,95 \% \mathrm{CI}$, $0.22-0.81)$, although large inconsistency $\left(I^{2}=80 \%\right)$ remained. Similar to the current review, variability in study populations, measurements of the intervention and exposure, and statistical analyses, likely contributed to significant heterogeneity in the models. In particular, the authors graded the overall quality of the evidence as "low" due to considerable limitations observed in the primary studies. Potential confounding of results from historical threats to internal validity, especially the introduction of antiretroviral medicines that minimize transmissible viral load and sexual health promotion programs, was a noted concern. Additionally, the statistical power to detect a significant result was low due to the fact that HIV seroconversions were a relatively rare event, which is also a problem in studies examining the role of NEP in preventing $\mathrm{HCV}$ seroconversion. Of note, many of the primary studies only examined HIV incidence as a secondary outcome.

\section{Implications for research and practice}

The previously discussed qualitative between-study differences that may be contributing to the substantial statistical heterogeneity and large inconsistency raise several important implications for future research. More specifically, there is a need for well-designed cohort studies that follow seronegative individuals forward in time to track potential seroconversion. It is suggested that these studies seek standardization of interventions and outcomes in the following areas: (1) inclusion criteria, (2) injection use timeframe, (3) definition and measurement of NEP use, (4) outcome assessment, and (5) statistical analysis plan.

With regard to suggestion 3, more objective measures of program attendance are recommended, but may be difficult to implement in practice. In particular, some needle exchanges do not require identification to obtain needles [57], which precludes the ability to objectively track program attendance. Alternatively, a randomly assigned identification number could be implemented to track both program attendance and any potential seroconversion.

With regard to suggestion 5, given the fact that a recent meta-analysis found a $94 \%$ increased risk of $\mathrm{HCV}$ seroconversion among injection drug users who shared syringes (pooled risk ratio $=1.94,95 \%$ confidence interval (CI) $1.53,2.46)$ [58], the sharing of syringes is an important covariate that should be standardized, measured, and adjusted for in future studies. Although a recent report by the US Centers for Disease Control (CDC) suggested that NEPs can reduce the sharing of syringes, White PWIDs, who are largely driving the HCV epidemic in nonurban areas, had the highest rate of syringe sharing [59].

Importantly, merely providing clean needles to PWIDs may not be enough to prevent new cases of HCV. Crofts et al. [60] first documented new $\mathrm{HCV}$ infections in PWIDs who reported no needle sharing, which suggests that $\mathrm{HCV}$ infection could be spread in other ways, such as sharing of contaminated equipment (e.g., mixing spoons and filters). Furthermore, a recent study conducted with heroin injectors in Denver, Colorado, observed barriers to using a clean needle for every injection, such as being in withdraw and fear of arrest that may prevent the use of a clean needle for every injection [61]. However, research examining these barriers in rural settings is nonexistent and represents an area ripe for inquiry.

Given the mixed findings and substantial heterogeneity and inconsistency observed in both this review and previous reviews, there is insufficient empirical evidence to either recommend or discount NEP for the prevention of HCV. However, despite this mixed evidence, the US CDC recently recommended implementation of these programs in rural areas that have been disproportionately affected by the recent opioid and heroin epidemics [59]. Unfortunately, not much is currently known regarding the experience of opening these programs in rural areas. Therefore, research elucidating the unique context in which these programs are implemented in rural areas, as well as the challenges and barriers experienced, is needed. To the best of our knowledge, there are currently only nine programs listed in the North American Syringe Exchange Network that are located in Central Appalachia (two programs in Kentucky, one in Tennessee, 
none in Virginia, and six in West Virginia) [62]. However, this total represents eight additional programs that have opened since June, 2014, when only one program operated in Nashville Tennessee [63].

\section{Strengths}

There are at least six potential strengths of the current meta-analysis. First, to the best of the author's knowledge, this systematic review with meta-analysis represents the first work using meta-analytic methods to provide quantitative estimates of the impact of NEPs on the prevention of $\mathrm{HCV}$ in PWIDs since the work of Hagan et al. [12]. The recently published systematic review with meta-analysis by Sawangjit et al. [23] only focused on pharmacy-based NEP and HCV prevalence (as opposed to the prevention of incident cases). Secondly, included studies were limited to designs which promote drawing causal inferences (i.e., cohort and case-control). Third, studies were not limited to the injection of illegal drugs. Fourth, these mixed results are consistent with previous studies. Fifth, the use of an objective serological measure of the outcome minimized potential bias in the reporting of HCV status. Sixth, this review has led to specific recommendations for the design of studies to minimize between-study heterogeneity and inconsistency, which may be preventing definitive conclusions regarding the effect of NEPs on the prevention of HCV.

\section{Limitations}

There are at least six potential limitations to be considered when reviewing the results of this meta-analysis. First, the current study excluded SIFs and pharmacies from the search due to our objective of examining the evidence related to programs that both collect and distribute (i.e., exchange) needles. In contrast, SIFs primarily provide clean needles for the injection of drugs on-site under medical monitoring. However, it has been noted that SIFs may have an important role in preventing $\mathrm{HCV}$ infection among PWIDs by serving as an additional mechanism for the provision of sterile needles in addition to NEPs [64]. Therefore, our results are limited to only one mechanism of sterile needle access. In addition to sterile needle provision, SIFs may greatly reduce risky injection practices (i.e., syringe sharing) that lead to HCV infection [64]. Unfortunately, SIFs are not yet widely available in the USA, in general, and in the rural areas of the USA that are in the midst of the HCV epidemic, in specific. The very first SIFs in the USA are preparing to open in 2017 in an urban location on the West Coast of the USA [65]. Current evidence regarding their impact on $\mathrm{HCV}$ seroconversion is lacking. Hagan et al. [12], upon which we based our search strategy, failed to find any articles describing the impact of SIFs or pharmacy sales on HCV seroconversion that met their inclusion criteria for their systematic review with meta-analysis. There were 15 studies among the 555 included in this systematic review that described SIFs. Only two of these studies obtained an objective measure (i.e., serum or saliva) of HCV infection. However, both studies were cross-sectional and did not provide data that would allow calculation of the association between SIF use and $\mathrm{HCV}$ infection. Similarly, and as discussed above, there is very limited evidence ( $N=2$ studies) that currently exists on the impact of pharmacy-based NEP, an area ripe for further inquiry. Secondly, the current review was unable to formally assess differential study effects stemming from different designs using moderator analysis due to the small sample size. Third, the weaknesses and potential biases inherent in individual studies are included in a metaanalysis, which may have negatively affected this study's ability to detect significant results. Such biases include information bias that could have been present from selfreports of injection status and NEP attendance, as well as volunteer bias $[66,67]$, which represents the phenomenon of NEP attendance by PWIDs that may be at higher risk for infectious disease. Fourth, it is possible that studies were missed during the systematic review and not included in the meta-analysis. In addition to not searching for unpublished sources, the fact that over 100 full text articles had to be reviewed to assess inclusion and exclusion criteria may indicate that studies that address this topic are not well described in either the title or abstract. Fifth, a small number of included studies precluded a complete assessment of the possibility of small study effects, including publication bias. Finally, because the aggregate data approach for this meta-analysis was used, these results are subject to ecological fallacy [68].

\section{Conclusions}

The impact of NEP on the prevention of HCV in PWIDs remains unclear. Such lack of clarity is likely due to substantial between-study heterogeneity in study design, inclusion criteria, intervention definition, outcome assessment, and statistical analyses that yield different pooled results depending on whether or not a time component (hazard ratio) is included in the analysis. Studies examining the operation of NEPs in rural areas are particularly needed, along with research examining the unique barriers to using clean needles experienced by PWIDs, to clarify the overall contribution of the presence of clean needles in the environment obtained from NEPs to the successful prevention of new cases of HCV. Future studies should also examine the impact of other sources of clean needles available for injection, such as SIFs and pharmacies, on the prevention of HCV infection in PWIDs. Given the potential benefits of NEP for reducing infectious disease in a population, future studies incorporating standardized populations, interventions, comparisons, outcomes, and analyses are critically needed to inform public health practice and policy. 


\section{Additional files}

Additional file 1: Search strategy examples. This document contains the queries entered into each database. (PDF $236 \mathrm{~kb}$ )

Additional file 2: Screening list and codebook. This spreadsheet contains the reasons for inclusion and exclusion for all screened abstracts and articles, and the codebook for included articles. (XLSX 147 kb)

\section{Abbreviations}

HCV: Hepatitis C virus; HIV: Human immunodeficiency virus; HR: Hazard ratio; NEP: Needle exchange program; NNR: Number needed to read; NOS: NewcastleOttawa Quality Assessment Scale; OR: Odds ratio; PRISMA: Preferred Reporting Items for Systematic Reviews and Meta-analyses; PWID: People who inject drugs; SIF: Supervised injection facility

\section{Acknowledgements}

Not applicable.

\section{Funding}

Research reported in this publication was supported by the National Institute of General Medical Sciences of the National Institutes of Health under award number U54GM104942. The content is solely the responsibility of the authors and does not necessarily represent the official views of the National Institutes of Health.

\section{Availability of data and materials}

All data generated or analyzed during this study are included in this published article and its supplementary information files.

\section{Authors' contributions}

SMD conceived the study topic, designed the study, acquired the data, performed the main data analyses, and drafted the manuscript. SD independently acquired the data as a second reviewer and assisted with drafting the manuscript. ALK assisted with drafting the manuscript, interpretation of the data, and provided critically important intellectual content during manuscript revisions. GAK provided expert advice regarding the study design and analyses, assisted with drafting the manuscript, and provided critically important intellectual content during manuscript revisions. $K Z, A B$, and $D D$ assisted with interpretation of the data and provided critically important intellectual content during manuscript revisions. MF provided expert advice regarding the study design, assisted with interpretation of the data, and contributed important intellectual content during manuscript revisions. All authors read and approved the final manuscript and take public responsibility for their contributions to the manuscript.

\section{Competing interests}

The authors declare that they have no competing interests.

\section{Consent for publication}

Not applicable.

\section{Ethics approval and consent to participate}

Not applicable.

\section{Publisher's Note}

Springer Nature remains neutral with regard to jurisdictional claims in published maps and institutional affiliations.

\section{Author details}

'School of Medicine, Department of Emergency Medicine, Robert C. Byrd Health Sciences Center, West Virginia University, PO Box 9149, Morgantown, W 26506-9149, USA. ²Department of Social and Behavioral Sciences, West Virginia University, PO Box 9190, Morgantown, WV 26506, USA. ${ }^{3}$ Department of Biostatistics, West Virginia University, PO Box 9190, Morgantown, WV 26506, USA. ${ }^{4}$ Department of Medicine, Section of Infectious Diseases, West Virginia University, PO Box 9163, Morgantown, WV 26506, USA.
Received: 18 February 2017 Accepted: 8 May 2017

Published online: 17 May 2017

\section{References}

1. Thrift AP, El-Serag HB, Kanwal F. Global epidemiology and burden of HCV infection and HCV-related disease. Nat Rev Gastroenterol Hepatol. 2016 Dec 7. doi: 10.1038/nrgastro.2016.176. [Epub ahead of print] Review

2. Ward JW. The hidden epidemic of hepatitis C virus infection in the United States: occult transmission and burden of disease. Top Antivir Med. 2013; 21(1):15-9.

3. Razavi H, Elkhoury AC, Elbasha E, Estes C, Pasini K, Poynard T, et al. Chronic hepatitis C Virus (HCV) disease burden and cost in the United States. Hepatology. 2013:57:2164-70.

4. Ly KN, Xing J, Klevens RM, Jiles RB, Ward JW, Holmberg SD. The increasing burden of mortality from viral hepatitis in the United States between 1999 and 2007. Ann Intern Med. 2012;156(4):271-8. Ann Intern Med. 2012 Jun 5; 156(11):840.

5. Rein DB, Wittenborn JS, Weinbaum CM, Sabin M, Smith BD, Lesesne SB. Forecasting the morbidity and mortality associated with prevalent cases of pre-cirrhotic chronic hepatitis C in the United States. Dig Liver Dis. 2011; 43(1):66-72. Epub 2010 Jun 17

6. Galbraith JW, Donnelly JP, Franco RA, Overton ET, Rodgers JB, Wang HE. National estimates of healthcare utilization by individuals with hepatitis $C$ virus infection in the United States. Clin Infect Dis. 2014:59(6):755-64. Epub 2014 Jun 9.

7. Averhoff FM, Glass N, Holtzman D. Global burden of hepatitis C: considerations for healthcare providers in the United States. Clin Infect Dis. 2012;55 Suppl 1:S10-5. doi:10.1093/cid/cis361.

8. Centers for Disease Control and Prevention (CDC). Viral hepatitis statistics and surveillance. Surveillance for Viral Hepatitis-United States 2014. Available at:. Accessed August 26th, 2016 DC

9. Page K, Morris MD, Hahn JA, Maher L, Prins M. Injection drug use and hepatitis $C$ virus infection in young adult injectors: using evidence to inform comprehensive prevention. Clin Infect Dis. 2013;57 Suppl 2:S32-8

10. Strathdee SA, Beyrer C. Threading the Needle-how to stop the HIV outbreak in rural Indiana. N Engl J Med. 2015;24 [Epub ahead of print]

11. HealthyPeople.gov. 2020 Topics \& Objectives. Immunization and infectious diseases. Available at: http://www.healthypeople.gov/2020/topics-objectives/ topic/immunization-and-infectious-diseases/objectives. Accessed 29 Oct 2015

12. Hagan $H$, Pouget ER, Des Jarlais DC. A systematic review and meta-analysis of interventions to prevent hepatitis $C$ virus infection in people who inject drugs. J Infect Dis. 2011;204(1):74-83.

13. Jordan AE, Jarlais DD, Hagan H. Prescription opioid misuse and its relation to injection drug use and hepatitis C virus infection: protocol for a systematic review and meta-analysis. Syst Rev. 2014;3(1):95 [Epub ahead of print].

14. Suryaprasad AG, White JZ, Xu F, Eichler BA, Hamilton J, Patel A, et al. Emerging epidemic of hepatitis $C$ virus infections among young non-urban persons who inject drugs in the United States, 2006-2012. Clin Infect Dis. 2014:59(10):1411-9.

15. Havens JR, Lofwall MR, Frost SD, Oser CB, Leukefeld CG, Crosby RA. Individual and network factors associated with prevalent hepatitis $C$ infection among rural Appalachian injection drug users. Am J Public Health. 2013;103(1):e44-52. Epub 2012 Nov 15.

16. Thursz $\mathrm{M}$, Fontanet $\mathrm{A}$. HCV transmission in industrialized countries and resource-constrained areas. Nat Rev Gastroenterol Hepatol. 2014;11(1):28-35. doi:10.1038/nrgastro.2013.179. Epub 2013 Oct 1.

17. Alter MJ. HCV routes of transmission: what goes around comes around. Semin Liver Dis. 2011:31(4):340-6. doi:10.1055/s-0031-1297923. Epub 2011 Dec 21.

18. Nelson PK, Mathers BM, Cowie B, Hagan H, Des Jarlais D, Horyniak D, Degenhardt L. Global epidemiology of hepatitis B and hepatitis C in people who inject drugs: results of systematic reviews. Lancet. 2011;378(9791):571-83. doi:10.1016/S0140-6736(11)61097-0. PubMed PMID: 21802134, PubMed Central PMCID: PMC3285467, Review.

19. Hedrich D, Hartnoll R, et al. Harm reduction interventions. In: N. el-Guebaly. In: Textbook of addiction treatment: international perspectives. edsth ed. 2015. p. 1291-313. doi:10.1007/978-88-470-5322-9_133.

20. Centers for Disease Control and Prevention. Syringe services programs. Available at: https://www.cdc.gov/hiv/risk/ssps.html. Accessed 17 Apr 2017.

21. MacArthur Gl, van Velzen E, Palmateer N, Kimber J, Pharris A, Hope V, et al. Interventions to prevent HIV and hepatitis $C$ in people who inject drugs: a review of reviews to assess evidence of effectiveness. Int J Drug Policy. 2014;25(1):34-52. doi:10.1016/j.drugpo.2013.07.001. Epub 2013 Aug 21. 
22. Abdul-Quader AS, Feelemyer J, Modi S, Stein ES, Briceno A, Semaan S, et al. Effectiveness of structural-level needle/syringe programs to reduce HCV and HIV infection among people who inject drugs: a systematic review. AIDS Behav. 2013;17(9):2878-92. doi:10.1007/s10461-013-0593-y.

23. Sawangjit R, Khan TM, Chaiyakunapruk N. Effectiveness of pharmacy-based needle/syringe exchange program for people who inject drugs: a systematic review and meta-analysis. Addiction. 2016. doi:10.1111/add. 13593. [Epub ahead of print] Review.

24. Nacopoulos AG, Lewtas AJ, Ousterhout MM. Syringe exchange programs: impact on injection drug users and the role of the pharmacist from a U.S. perspective. J Am Pharm Assoc (2003). 2010;50(2):148-57. doi:10.1331/JAPhA.2010.09178.

25. Moher D, Liberati A, Tetzlaff J, Altman DG; PRISMA Group. Preferred reporting items for systematic reviews and meta-analyses: the PRISMA statement. PLoS Med. 2009 Jul 21;6(7):e1000097. doi: 10.1371/journal.pmed. 1000097. Epub 2009 Jul 21.

26. Higgins JPT, Green S (editors). Cochrane Handbook for Systematic Reviews of Interventions Version 5.1.0 [updated March 2011]. The Cochrane Collaboration, 2011. Available from http://handbook.cochrane.org/. Accessed 24 March 2016.

27. Potier C, Laprévote V, Dubois-Arber F, Cottencin O, Rolland B. Supervised injection services: what has been demonstrated? A systematic literature review. Drug Alcohol Depend. 2014;145:48-68. doi:10.1016/j.drugalcdep. 2014.10.012. Epub 2014 Oct 23. Review.

28. Schlicting EG, Johnson ME, Brems C, Wells RS, Fisher DG, Reynolds G. Validity of injecting drug users' self report of hepatitis A, B, and C. Clin Lab Sci. 2003;16(2):99-106.

29. Stern RK, Hagan H, Lelutiu-Weinberger C, Des Jarlais D, Scheinmann R, Strauss S, et al. The HCV Synthesis Project: scope, methodology, and preliminary results. BMC Med Res Methodol. 2008;8:62. doi:10.1186/1471-2288-8-62.

30. van Driel ML, DeSutter A, DeMaeseneer J, et al. Searching for unpublished trials in Cochrane reviews may not be worth the effort. J Clin Epidemiol. 2009;62(8):838-44.

31. Lee E, Dobbins M, Decorby K, McRae L, Tirilis D, Husson H. An optimal search filter for retrieving systematic reviews and meta-analyses. BMC Med Res Methodol. 2012;12:51.

32. Kali A, Srirangaraj S. EndNote as document manager for summative assessment. J Postgrad Med. 2016 Jan 15. doi: 10.4103/0022-3859.174158. [Epub ahead of print]

33. Corporation M. Microsoft Excel (2013). Redmond, Washington: Microsoft Corporation; 2013

34. Wells, GA, Shea, B, O'Connell, D, Peterson, J, Welch, V, Losos, M, et al. The Newcastle-Ottawa Scale (NOS) for assessing the quality of nonrandomised studies in meta-analyses. Available at: http://www.ohri.ca/programs/clinical_ epidemiology/oxford.asp. Accessed 19 Sept 2016.

35. Ahn S, Becker BJ. Incorporating quality scores into meta-analysis. J Educ Behav Stat. 2011;36:555-85.

36. Dersimonian R, Laird N. Meta-analysis in clinical trials. Control Clin Trials. 1986;7:177-88

37. Higgins JP, Thompson SG, Deeks JJ, Altman DG. Measuring inconsistency in meta-analyses. BMJ. 2003;327(7414):557-60. Review.

38. Sterne JA, Sutton AJ, loannidis JP, Terrin N, Jones DR, Lau J, et al. Recommendations for examining and interpreting funnel plot asymmetry in meta-analyses of randomised controlled trials. BMJ. 2011;343:d4002. doi:10. 1136/bmj.d4002.

39. Biostat. Comprehensive meta-analysis. (3.0). Englewood, NJ: Biostat, Inc; 2016.

40. Hagan H, Des Jarlais DC, Friedman SR, Purchase D, Alter MJ. Reduced risk of hepatitis $B$ and hepatitis $C$ among injection drug users in the Tacoma syringe exchange program. Am J of Public Health. 1995;85(11):1531-7.

41. Hagan $H$, Thiede $H$, Des Jarlais DC. Hepatitis C virus infection among injection drug users: survival analysis of time to seroconversion. Epidemiology. 2004;15(5):543-9.

42. Holtzman D, Barry V, Ouellet L, Des Jarlais DC, Vlahov D, Golub ET, et al. The influence of needle exchange programs on injection risk behaviors and infection with hepatitis C virus among young injection drug users in select cities in the United States, 1994-2004. Prev Med. 2009;49(1):68-73.

43. Patrick DM, Tyndall MW, Cornelisse PG, Li K, Sherlock CH, Rekart ML, et al. Incidence of hepatitis $C$ virus infection among injection drug users during an outbreak of HIV infection. Can Med Assoc J. 2001;165(7):889-95.

44. Roy E, Alary M, Morissette C, Leclerc P, Boudreau JF, Parent R, et al. High hepatitis $C$ virus prevalence and incidence among Canadian intravenous drug users. Int J STD AIDS. 2007;18(1):23-7.
45. Todd CS, Nasir A, Stanekzai MR, Fiekert K, Sipsma HL, Vlahov D, et al. Hepatitis $C$ and HIV incidence and harm reduction program use in a conflict setting: an observational cohort of injecting drug users in Kabul. Afghanistan Harm Reduct J. 2015;12:22.

46. Thorpe LE, Ouellet LJ, Hershow R, Bailey SL, Williams IT, Williamson J, et al. Risk of hepatitis $C$ virus infection among young adult injection drug users who share injection equipment. Am J Epidemiol. 2002; 155(7):645-53.

47. Lamothe F, Bruneau J, Franco E, Vincelette J, Lachance N. Prevalence, incidence and risk factors for hepatitis $C$ infection among injection drug users participating in the Saint-Luc cohort. A report prepared under contract (5498) with the Laboratory Centre for Disease Control, Health Canada. Montreal, Canada: McGill University; 1997.

48. Zibbell JE, lqbal K, Patel RC, Suryaprasad A, Sanders KJ, Moore-Moravian L, et al. Increases in hepatitis $C$ virus infection related to injection drug use among persons aged $\leq 30$ years - Kentucky, Tennessee, Virginia, and West Virginia, 2006-2012. MMWR Morb Mortal Wkly Rep. 2015:64(17):453-8.

49. Beheshti A, Lucas L, Dunz T, Haydash M, Chiodi H, Edmiston B, et al. An evaluation of naloxone use for opioid overdoses in West Virginia: a literature review. Am Med J. 2015;6(1):9-13.

50. Chelleng PK, Borkakoty BJ, Chetia M, Das HK, Mahanta J. Risk of hepatitis C infection among injection drug users in Mizoram. India Indian J Med Res. 2008;128(5):640-6.

51. Garfein RS, Rondinelli A, Barnes RF, Cuevas J, Metzner M, Velasquez M, et al. HCV infection prevalence lower than expected among 18 to 40-year-old injection drug users in San Diego. Calif J Urban Health. 2013;90(3):516-28.

52. Hope V, Parry JV, Marongui A, Ncube F. Hepatitis C infection among recent initiates to injecting in England 2000-2008: is a national hepatitis C action plan making a difference? J Viral Hepatitis. 2012;19(1):55-64.

53. Panda S, Roy T, Pahari S, Mehraa J, Sharma N, Singh G, et al. Alarming epidemics of human immunodeficiency virus and hepatitis $C$ virus among injection drug users in the northwestern bordering state of Punjab, India: prevalence and correlates. Int J STD AIDS. 2014;25(8):596-606.

54. Plitt SS, Gratrix J, Hewitt S, Conroy P, Parnell T, Lucki B, et al. Seroprevalence and correlates of HIV and HCV among injecting drug users in Edmonton. Alberta Can J Pub Health. 2010;101(1):50-5.

55. Turner KM, Hutchinson S, Vickerman P, Hope V, Craine N, Palmateer N, et al. The impact of needle and syringe provision and opiate substitution therapy on the incidence of hepatitis C virus in injecting drug users: pooling of UK evidence. Addiction. 2011;106(11):1978-88.

56. Aspinall EJ, Nambiar D, Goldberg DJ, Hickman M, Weir A, Van Velzen E, et al. Are needle and syringe programmes associated with a reduction in HIV transmission among people who inject drugs: a systematic review and meta-analysis. Int J Epidemiol. 2014;43(1):235-48.

57. Sherman SG, Purchase D. Point Defiance: a case study of the United States' first public needle exchange in Tacoma. Washington Int J Drug Policy. 2001; 12(1):45-57.

58. Pouget ER, Hagan $H$, Des Jarlais DC. Meta-analysis of hepatitis C seroconversion in relation to shared syringes and drug preparation equipment. Addiction. 2012; 107(6):1057-65. doi:10.1111/j.1360-0443.2011.03765.x. Epub 2012 Apr 17.

59. Wejnert C, Hess KL, Hall HI, Van Handel M, Hayes D, Fulton P Jr, et al. Vital signs: trends in HIV diagnoses, risk behaviors, and prevention among persons who inject drugs - United States. MMWR Morb Mortal Wkly Rep. 2016 Dec 2;65(47):1336-1342. doi: 0.15585/mmwr.mm6547e1

60. Crofts N, Aitken C. Incidence of blood borne virus infection and risk behaviours in a cohort of injecting drug users in Victoria, 1990-1995. Med J Aust. 1997;167:17-20.

61. Phillips KT. Barriers to practicing risk reduction strategies among people who inject drugs. Addict Res Theory. 2016;24(1):62-8. Epub 2015 Jul 21.

62. North American Syringe Exchange Network. Directory of syringe exchange programs. Available at: https://nasen.org/directory/. Accessed 19 Apr 2017.

63. North American Syringe Exchange Network. Updated amfAR SSP coverage map. Available at: https://nasen.org/news/2014/aug/08/updated-amfarsyringe-service-program-coverage-map/. Accessed 19 Apr 2017.

64. Semaan S, Fleming P, Worrell C, Stolp H, Baack B, Miller M. Potential role of safer injection facilities in reducing HIV and hepatitis $C$ infections andoverdose mortality in the United States. Drug Alcohol Depend. 2011; 118(2-3):100-10. doi:10.1016/j.drugalcdep.2011.03.006. PubMed PMID: 21515001, Epub 2011 Apr 23. 
65. Ghorayshi A. The US is opening its first "Safe Injection Facility" for heroin addicts. Available at: https://www.buzzfeed.com/azeenghorayshi/safeinjection-space-heroin?utm_term=.fdV3bMd65\#.imv4D6O8M. Accessed 18 Apr 2017.

66. Hagan H, McGough JP, Thiede H, Hopkins SG, Weiss NS, Alexander ER. Volunteer bias in nonrandomized evaluations of the efficacy of needleexchange programs. J Urban Health. 2000;77(1):103-12.

67. Schechter MT, Strathdee SA, Cornelisse PG, Currie S, Patrick DM, Rekart ML, et al. Do needle exchange programmes increase the spread of HIV among injection drug users?: an investigation of the Vancouver outbreak. AIDS. 1999;13(6):F45-51.

68. Cooper H, Patall EA. The relative benefits of meta-analysis conducted with individual participant data versus aggregated data. Psychol Methods. 2009; 14(2):165-76. doi:10.1037/a0015565.

Submit your next manuscript to BioMed Central and we will help you at every step:

- We accept pre-submission inquiries

- Our selector tool helps you to find the most relevant journal

- We provide round the clock customer support

- Convenient online submission

- Thorough peer review

- Inclusion in PubMed and all major indexing services

- Maximum visibility for your research

Submit your manuscript at www.biomedcentral.com/submit
Biomed Central 


\subsection{Study 2 Manuscript}

Title: Qualitative Case Study of Needle Exchange Programs for the Prevention of Hepatitis C Virus in the Rural Appalachian Region of the United States

Authors: Stephen M. Davis (smdavis@hsc.wvu.edu), ${ }^{1}$ Danielle Davidov

(ddavidov@hsc.wvu.edu), ${ }^{12}$ Alfgeir L. Kristjansson (alkristjansson@hsc.wvu.edu), ${ }^{2}$ Keith Zullig (kzullig@hsc.wvu.edu),,2 Adam Baus (abaus@hsc.wvu .edu),,2 Melanie Fisher (mfisher@hsc.wvu.edu) ${ }^{3}$

Author Affiliations: 'Department of Emergency Medicine, West Virginia University, PO Box 9149, Morgantown, WV 26506, United States ${ }^{2}$ Department of Social and Behavioral Sciences, West Virginia University, PO Box 9190, Morgantown, WV 26506, United States ${ }^{3}$ Department of Medicine, Section of Infectious Diseases, West Virginia University, PO Box 9163, Morgantown, WV 26506, United States

Address for Correspondence: Stephen M. Davis, Adjunct Professor, School of Medicine, Department of Emergency Medicine, Robert C. Byrd Health Sciences Center, West Virginia University, PO Box 9149, Morgantown, WV, United States, 26506-9149, Office Phone: 304-293-1326, Fax: 304-293-6702, E-mail: smdavis@hsc.wvu.edu Funding: This research did not receive any specific grant from funding agencies in the public, commercial, or not-for-profit sectors.

Declaration of Interest: none.

Word Count: Abstract: 300; Text (excluding title page, abstract, references): 7,940 References: 56 
Title: Qualitative Case Study of Needle Exchange Programs for the Prevention of Hepatitis C Virus in the Rural Appalachian Region of the United States

Keywords: needle exchange programs; hepatitis C virus; people who inject drugs; paraphernalia laws; qualitative case study

\section{ABSTRACT}

Background. The rural Appalachian region of the United States is in the midst of a hepatitis $\mathrm{C}$ virus epidemic driven by injection of opioids, particularly heroin, with contaminated syringes. In response to this epidemic, several needle exchange programs (NEP) have opened to provide clean needles and other supplies and services to people who inject drugs (PWID). However, no studies have investigated the barriers and facilitators to implementing NEPs in rural areas.

Methods. This qualitative case study consisted of interviews with program directors, police chiefs, law enforcement members, and PWIDs affiliated with two NEPs in the rural state of West Virginia. Interview transcripts were coded inductively and analyzed using NVIVO® software. Final common themes related to barriers and facilitators of past program openings, current program operations, and future program plans, were derived through a consensus of two data coders.

Results. Both NEPs struggled to find existing model programs, but benefited from broad community support that facilitated implementation. The largest operational barrier was the legal conundrum created by paraphernalia laws that criminalize possession of syringes. However, both PWIDs and law enforcement appreciated the comprehensive 
services provided by these programs. Program location and transportation difficulties were additional noted barriers. Future program operations are threatened by funding shortages and bans, but necessitated by unexpected program demand.

Conclusion. Despite broad community support, program operations are threatened by growing volumes, funding shortages, and the federal government's prohibition on the use of funds to purchase needles. Paraphernalia laws create a legal conundrum in the form of criminal sanctions for the possession of needles, which may inadvertently promote needle sharing and disease transmission. Future studies should examine additional barriers to using clean needles provided by rural NEPs that may blunt the effectiveness of NEPs in preventing disease transmission, and elucidate the factors that contribute to geographical funding disparities.

\section{INTRODUCTION}

Hepatitis $\mathrm{C}$ virus (HCV) is a blood-borne infection that is commonly transmitted during injection drug use (Keeshin \& Feinberg, 2016; Ward, 2013). HCV affects an estimated 177.5 million (2.5\%) of the world's population (Petruzziello, Marigliano, Loquercio, Cozzolino, \& Cacciapuoti, 2016), and is the leading cause of advanced liver diseases (Cooke et al., 2013; Stepanova \& Younossi, 2017). The worldwide economic burden associated with HCV-related liver disease is enormous and includes both direct costs related to medical care and indirect costs such as loss of work productivity (Estes et al., 2015; Stepanova \& Younossi, 2017). Even in low HCV prevalence $(<1 \%)$ countries such as Iran, HCV economic burden is estimated to eclipse more than $\$ 1$ 
billion per year (Stepanova \& Younossi, 2017; Zare, Fattahi, Sepehrimanesh, \& Safarpour, 2016).

Although most countries have seen rates of HCV plateau (Thrift, El-Serag, \& Kanwal, 2016), incident cases of HCV infection increased more than 2.9 fold in the United States between 2010 and 2015 (CDC, 2017). The majority of these new infections have been observed in white, young, people who inject drugs (PWIDs) residing in nonurban areas of the United States (CDC, 2017; Havens et al., 2013; Page, Morris, Hahn, Maher, \& Prins, 2013; Suryaprasad et al., 2014; Ward, 2013; Zibbell et al., 2015). Between 2006 and 2012, the rural, central Appalachian region of the United States (Kentucky, Tennessee, Virginia, and West Virginia) observed a 364\% increase in acute HCV cases in young persons ( $\leq 30$ years old) (Zibbell et al., 2015). There is evidence that this exponential increase is highly correlated with a regional prescription opioid abuse epidemic and an increase in injection of illicit street drugs, particularly heroin, that occurred during the same time period (Beheshti et al., 2015; Zibbell et al., 2015; Zibbell et al., 2018).

The rural state of West Virginia, located entirely in the Appalachian region, has the second-highest rate of incident cases of HCV in the country (3.4 per 100,000)(CDC, 2017) and shares many of the demographic characteristics of PWIDs who are thought to be driving the US epidemic (i.e., white and young). Between 2007 and 2015, the HCV incidence rate increased over $300 \%$ in West Virginia, and the most frequently reported risk factors for developing acute HCV infection were injection drug use and used street drugs (WV DHHR, 2017). In response to this epidemic, needle exchange programs (NEPs) began opening in the state during 2015. 
NEPs exchange used needles for clean needles, and have been shown to prevent HCV among PWIDs in several studies (MacArthur et al., 2014). NEPs are recommended by the World Health Organization and other global bodies as a mechanism to prevent transmission of infectious diseases including HCV (Csete et al., 2016). In addition to clean needles, NEPs also provide other injection equipment supplies, infectious diseases testing, medical care, and linkage to substance abuse treatment (Stancliff, Phillips, Maghsoudi, \& Joseph, 2015). However, in the United States, NEPs remain controversial (Csete et al., 2016; Tempalski et al., 2007). Such controversy stems from a historical tendency to treat drug abuse as a moral problem that requires a criminal response in the form of arrests and other sanctions (Csete et al., 2016; Des Jarlais, 2017; Vlahov et al., 2001). Furthermore, some members of the public have voiced concern that NEPs amount to explicit encouragement and support of destructive drug use (Vlahov et al., 2001). Consequently, public policies have limited the coverage and effectiveness of NEPs (Csete et al., 2016; Des Jarlais, 2017) in the urban areas where they historically operated.

Despite the clear need for NEPs in rural states like West Virginia, there remains a dearth of research documenting the unique context and challenges to operating NEPs in rural areas that can provide critical information on the barriers and facilitators that impact overall program effectiveness (Davis et al., 2017). A mail/telephone survey with the directors of NEPs operating in the United States in 2013 revealed that rural programs were more likely to report experiencing a lack of resources/funding compared to suburban and urban programs (73\% versus $64 \%$ and $63 \%$, respectively) (Des Jarlais et al., 2015). Rural programs were also slightly more likely to report experiencing 
problems reaching or recruiting participants compared to urban programs $(20 \%$ versus $18 \%$, respectively). However, challenges and barriers related to program design, implementation, and sustainability were not reported. Therefore, the purpose of this study was to conduct a qualitative case study of two NEPs that opened in West Virginia in 2015. As part of this case study, we compared and contrasted the experiences and contexts of each program to identify common themes related to program operations. Of specific interest were the facilitators and barriers to the implementation and ongoing operations of each program. In particular, this case study sought to answer the following questions: (1) What were the facilitators and barriers to opening the program?; (2) What were the facilitators and barriers encountered during the day-to-day running of the program?; (3) What were the future plans for the program?; and, (4) What were the challenges and barriers related to these future plans?

\section{METHODS}

\section{Approach}

A multiple, intrinsic case study approach was selected for the following reasons: 1) each case (NEP) was of interest in and of itself (i.e., intrinsic) (Stake, 1995); 2) the goal of this study was to obtain an in-depth understanding of each case (Creswell, 2013); and, 3) each program (case) was a bounded system (Creswell, 2013). For the purposes of this study, we bounded each case to the specific program, and examined the past, present, and future time orientations of each program. Although no specific time limits were placed on each orientation, all information gathered was focused on facilitators and barriers encountered by (or expected to be encountered by) the program during each timeframe. 


\section{Sampling}

Each case was selected using a purposive sampling strategy (Miles, 1994). West Virginia currently has 10 NEPs registered with the North American Syringe Exchange Network (North American Syringe Exchange Network, 2018). For this study, we purposefully selected two of the longest operating programs, a NEP operating within a free healthcare clinic and a NEP operating within a health department, to glean important insights from their significant experiences and document variations between the two program structures.

\section{Data collection}

To answer the central research questions, the lead author (SD) conducted indepth interviews with the director of each program. At one site, other administrative personnel (i.e., the nursing director and site administrator) also participated in the interview. In-depth interviews were selected to obtain a deeper understanding of these novel programs that arose in response to a desperate situation. In the interest of triangulating data to develop emergent themes, police chiefs and other law enforcement officers in the areas served by the programs, as well as the NEP attendees currently using each program, were interviewed. Director interviews occurred in March 2016, law enforcement interviews occurred during the summer of 2016, and interviews with program attendees occurred during the summer of 2017.

Prior to conducting each interview, a semi-structured interview guide was developed. The structure of the interview guide was focused on the facilitators and barriers encountered during the past and present, as well as those anticipated to occur 
in the future. However, due to the interest in obtaining an in-depth understanding of the central research questions from the viewpoint of respondents, each interview was semistructured, and conversations were allowed to emerge and flow in naturalistic directions that may have departed from the interview guide. This approach is consistent with a social constructivist framework (Denzin, 2011). Importantly, the specific questions included in the interview guide were also guided by initial discussions with each director and included questions of interest to him or her. Finally, a case study of the first NEP in the United States, Point Defiance (Sherman \& Purchase, 2001), was also used as a source of potential questions.

Prior to starting the interview, interviewees were informed that the discussion was voluntary and could be ended at any time without penalty. Interviewees were also informed that all discussions were confidential and that the specific program would not be identified in any publications or presentations. Therefore, each case has been assigned a pseudonym (Free Clinic NEP and Health Department NEP).

Each interview took place in a private conference room or via telephone and lasted approximately 60 minutes. Interviews were recorded using a TasCAM DR-05® stereo handheld microSD (TASCAM, 2016). As a backup measure, interviews were also recorded using Apple ${ }^{\circledR}$ iPhone's Voice Memos utility (Macworld, 2016). At various points throughout each interview, the interviewer summarized the discussion and sought clarification from the interviewee. 


\section{Data analysis}

Interviews were transcribed by a professional and entered into $\mathrm{NIVIO} \otimes 11$ Pro software for analysis (NVIVO, 2016). Prior to formal analysis, each transcript was read several times and notes were made regarding initial codes and categories. The initial codebook had a priori codes for past, present, and future, in which information on barriers and facilitators corresponding to each time period was categorized. However, no other codes were assigned a priori, and line by line coding was conducted with the objective of identifying approximately 5 to 7 general themes in the interest of parsimony. In the constructivist tradition, codes were allowed to emerge from the data prior to categorization into themes (Denzin, 2011). This approach allowed for the discovery of information that was surprising and not expected. As suggested by Yin, a cross case synthesis was conducted to denote similarities, differences, and emerging themes between the two cases (Yin, 2016).

After initial coding and classification, intensive group discussion with a second coder with expertise in qualitative research (DD) with a goal of simple consensus was employed to finalize the codes and overall themes (Brinkmann, 2015; Harry, 2005; Sandelowski, 2007). If needed, discrepancies were resolved by a third party. This approach is recommended by several methodologists in order to preserve the interpretive process at the core of qualitative analysis (Saldana, 2016), and is consistent with current case study practice (Atchan, Davis, \& Foureur, 2016), in specific, and generally accepted qualitative research reporting criteria (Tong, Sainsbury, \& Craig, 2007). Themes were classified according to time orientation (i.e., past, present, future) and whether it reflected a barrier or facilitator to program operations. Respondent 
quotes that captured the essence of each theme were selected as the primary data outcomes. Findings and interpretations of the data were shared with interviewees (i.e., member checking) to assess the accuracy (validity) of the measurement, and naturalistic generalizations were made by comparing emergent themes with previously published literature (Creswell, 2013). This protocol was approved by the referent university's Institutional Review Board.

\section{FINDINGS}

\section{Sample Characteristics}

Qualitative interviews were conducted with two program directors, a program administrator, a program nurse, two chiefs of police, two law enforcement officers, and eight PWIDs (4 male, 4 female) who attended the two programs.

\section{Description of Cases}

\section{Free Clinic NEP}

The Free Clinic was founded in 1984 by a group of concerned citizens on the premise that healthcare is a universal right that should be available to all citizens regardless of ability to pay. In response to an ever-growing need, the clinic has moved several times, occupying larger quarters each time, and currently occupies a two-story building with ten exam rooms, two waiting rooms, a medication room, conference rooms, offices, and storage areas. The agency has a staff of 21 , a volunteer corps of more than 200 , and provides more than 28,000 patient encounters and dispenses free medications in the millions to qualified patients every year. In addition to medication assistance, the Free Clinic provides primary health care with a professional staff of 
physicians, nurse practitioners, physician assistants, registered nurses (RNs), licensed practical nurses (LPNs), medical assistants, social workers, and therapists. Specialty clinics for various diseases (e.g., diabetes) and topics (e.g., women's health) are also offered. Some dental care and mental health services are provided on a limited basis. It is supported by the United Way, as well as various federal, state, and local grants, but also greatly depends on donations and in-kind gifts from individuals. All health care, including prevention, health awareness, and chronic disease management, are free of charge to qualified patients and are offered in clinics or in group education.

\section{Precipitating Event/Program Impetus}

Although Free Clinic personnel were aware that some patients were also injection drug users, the significant incidence of $\mathrm{HCV}$ among this population was not discovered until the Centers for Disease Control (CDC) updated its HCV screening guidelines to recommend testing for all persons between 45 and 64 years of age (Smith et al., 2012). The Free Clinic began implementation of the updated guidelines in June 2013, and within one month, slightly over $10 \%$ of 300 patients tested positive for HCV. This event led to an examination of factors related to the high HCV rate, which revealed that most of the HCV positive individuals either had a history of injection drug use or were currently injecting. Around the same time, clinic personnel became aware of a heroin epidemic in West Virginia that emerged from an opioid abuse epidemic (Beheshti et al., 2015). Collectively, these two factors led to the further exploration of a NEP to prevent new HCV infections. Between July 2013 and October 2014, the Free Clinic conducted in-depth research regarding the startup and implementation of NEPs, and 
presented this information to the Board of Directors at the October meeting in 2014. The board unanimously approved (20-0) the implementation of a NEP, which led to a quest to secure funding and logistical planning related to implementation. After obtaining support from the board to open a NEP, the Free Clinic NEP established a Community Advisory Board (CAB) comprised of members of the community, a representative of the police department, and a social worker. The $C A B$ also sought information from current users to guide the decision-making process. The Free Clinic NEP officially opened on August 19, 2015 and treated two clients the first day.

\section{Program Operations}

The Free Clinic NEP is open between 3:30 PM and 6:30 PM every Wednesday, and makes special arrangements for attendees who cannot attend on Wednesdays. During this time period, no other clinic services are provided. There are three staff members that are dedicated to the NEP, and students from a nearby university also assist with operations. PWIDs of all ages are accepted with a very specialized protocol for those under 18 years of age, although no children had yet been seen at the time of the interview. The average age of attendees is 33 (range 19-70) with slightly more males than females ( $60 \%$ versus $40 \%$, respectively). All but $3 \%$ of attendees are Caucasian. Each attendee is assigned a unique identification number for tracking purposes during the initial intake. Basic information regarding injection history, including duration and drug of choice, is also collected at this time. Clients are queried regarding whether or not they know their human immunodeficiency virus (HIV) and HCV status, and testing is encouraged. Importantly, all data collected in the context of the NEP is kept entirely separate from clinic medical records. The program operates year- 
round. At the time of data collection, 200 unduplicated patients had attended the exchange since its opening.

Each attendee leaves with approximately 30 to 50 clean needles. Additional drug paraphernalia, including cookers, cotton balls, alcohol swabs, and tourniquets, are also provided. The exchange offers rapid HCV testing as well as serum HIV testing. Attendees also have access to a social worker and a nurse practitioner to discuss any health-related issues. Discussions regarding harm reduction, healthcare, treatment, housing, and general case management are common.

\section{Health Department NEP}

The Health Department NEP is located within a public health agency that provides a plethora of environmental and epidemiological public health services including environmental services (e.g., food safety, etc.), clinic services (e.g., sexually transmitted infection (STI) clinics, immunizations, etc.), threat preparedness, and disease investigation.

\section{Precipitating Event/Program Impetus}

In November 2014, a local Office of Drug Control Policy was created by the mayor of the town in which the health department is located. An investigation by this office into the problem of drug use found an extremely high rate of drug overdose, primarily from heroin. However, most concerning was the observed rising death rate:

We had a very, very, very high rate of overdose and what was happening that grabbed our attention was our death rate. Our death rate was beginning to balloon. I don't remember the exact numbers but we had somewhere in the neighborhood of 15 deaths in January and February [2015] and it was continuing to rise at that time. (Administrator) 
During this same time period in 2015, Scott County, Indiana was in the midst of an HIV epidemic from injection of illegal drugs with contaminated needles that led to the opening of a NEP to combat the epidemic (Strathdee \& Beyrer, 2015). These two factors led to the decision to explore the implementation of a NEP in this local West Virginia community. After extensive research on other NEPs (including visits to a small rural community in a neighboring state between January and April 2015), obtaining legal clearance, and the acquisition of adequate funding, the Health Department NEP formally opened on September 2, 2015. At the time of data collection, the NEP had seen 977 unique clients and exchanged 2,500 needles since its opening.

\section{Program Operations}

The Health Department NEP runs three exchange rooms on Wednesdays during a five hour period. In contrast to the Free Health Clinic NEP, other health department services are also offered during the same time period. Approximately 15 individuals comprised of seven staff members, pharmacy, nursing, and medical students, and recovery coaches are present during operating hours. On any given Wednesday, approximately 130 attendees use the exchange with a single day high-volume of 144 attendees observed. Attendees receive a maximum of 40 clean needles, and most attendees bring in approximately 30 to 40 used needles to exchange. In addition to exchanging needles, the Health Department NEP provides recovery coaches, STI testing, as well as testing for HCV (serum) and HIV. Attendees are also queried regarding their infectious disease status, and are provided access to nurses who can examine their injection sites for potential infection. Teaching regarding safe and clean injection is also provided, and attendees with infected sites are seen by a nurse 
practitioner. The administrative staff described difficulties related to the fact that routine health department services are offered during the times that the NEP is open. For example, many of the NEP attendees were smokers who were frequently going outside to smoke, which disrupted other health department clientele.

Attendees of the Health Department NEP are heterogeneous and contain a range of individuals from functioning addicts that are gainfully employed despite addiction to one or more substances to the more stereotypical drug addict (e.g., unemployed, homeless, etc.). Although identification is not required by the Health Department NEP, the created ID number assigned to each participant for tracking purposes includes birth year. Gender is split evenly, and $96 \%$ of attendees are Caucasian, which is similar to West Virginia as a whole (94\% Caucasian) (U.S. Census Bureau, 2017). The average age is 37 and ranges from 21 to the 60 s.

Past Barriers and Facilitators to Opening the Program

\section{One Size Doesn't Fit All}

The theme, One Size Doesn't Fit All, was identified from NEP directors' descriptions of difficulties (i.e., barriers) encountered with locating an existing exchange program to serve as a model for the development of a NEP in their rural communities.

The Free Clinic NEP enlisted the support of students from a nearby university to conduct research on the existence of comparable exchange programs to no avail. This absence of model programs led to the need to modify elements of existing program policies from states such as New York to include policies for dealing with underage attendees and other unique aspects of the rural environment: 
So we did a lot of research and we found out that in some cities like Baltimore there's a syringe exchange on almost every corner at different times during the week. But we didn't find any information about small town USA. And l've also been involved in outreach in [another rural community] and have just recently did a conference call with several smaller communities. That's the key here. The smaller communities are trying to break into this but the only model that's out there is really for more urban areas. (Director)

Similarly, although the Health Department NEP was able to visit a program that was implemented in a nearby small community of approximately 5,000 residents, this program only exchanged needles and provided pamphlets with information, as opposed to the more comprehensive program models that include a variety of health services offered in other, more urban settings. Some NEP policies and procedures information was located on the Web, but the Health Department did not have the luxury of time to refine policies and procedures prior to program operations due to exponential program demand, which is further discussed below.

\section{Like a Good Neighbor}

The theme, Like a Good Neighbor, included participant descriptions of the facilitative role that support from the community played in both program implementation and the ability to sustain the program moving forward (discussed below). Despite difficulties related to finding model programs during program development, both program openings were significantly facilitated by support from the community, albeit in somewhat different ways.

The Free Clinic was initially concerned about a potential absence of community support and choose a silent opening that did not involve media announcements. However, once such announcements were made, the result was an overwhelming level of support: 
So we did a very quiet start. We did not publicize. ... . we knew that this would generate emotional feelings and thoughts and that we hoped people would call and ask questions and try their best to understand. The response was 14,000 dollars, and we did not ask for money.

In contrast, the Health Department NEP enjoyed broad community support due

to concern over the overwhelming number of opioid overdose deaths:

... [A] community meeting happened in March through a local, I don't know what you call her, just a local community member that was interested and 500 people attended that. . . This was organized as a Facebook campaign by a community person. This was not organized by any official. They got the room at the library and there were 500 people up there and it was testimony after testimony from families who had lost people from people who are recovered from this addiction. And there was uniform support. And in fact it went beyond the uniform support for the effort to the question, "Why are you not doing this?" (Administrator)

However, as discussed below, having a continuous supply of clean syringes was identified as a shared barrier to future operations.

Present Barriers and Facilitators to Operating the Program

\section{The Legal Conundrum}

The theme, The Legal Conundrum, was identified from participant discussions of the impact of paraphernalia laws and policing behaviors on program operations and participation. Both programs cited the critical importance of law enforcement support to successful program implementation and operations. However, existing paraphernalia laws and the fact that injection of illicit substances is an illegal activity created a quandary for law enforcement officials regarding the best approach to the possession of drug paraphernalia that may threaten the harm reduction goal of the NEPs:

So the police officer who participates got permission from the chief of police to participate and then we, our medical director and myself went over and had a face to face meeting with our city police chief because we felt like we needed them on our side. . . we didn't want to get to a point where we were giving out syringes, they would arrest somebody, confiscate those syringes and then. . . basically harm reduction is now no longer available to the community. (Director) 
Law enforcement leadership provided significant support for the programs and proffered directives to not seize clean needles in certain circumstances:

What my instructions have been is if you catch somebody coming out of [the NEP] with a bag of clean needles, or somebody that comes out of a pharmacy, that's not really drug paraphernalia. It's got to be in conjunction with you know you've got the spoon, you've got the tie off, you know it's clear that that's what they're using it for. (Police Chief)

However, despite this crucial support, variations in the particular actions taken in the case of clean (unused) needles were recorded. For example, one law enforcement officer advised NEP attendees to not call attention to themselves by engaging in suspicious or criminal activity that may lead to the discovery of syringes and possible citation or arrest:

It's still drug paraphernalia...If you're stopped by law enforcement, it's not an exemption because you're not supposed to be out here walking the streets carrying your drug paraphernalia and that kind of stuff. You know like I said they know what they're dealing with and they know the actual laws for the use of. Yeah, don't draw attention to yourself and then you might get by.

Other officers felt that giving citations for syringes would be a mechanism for linking attendees to treatment via a law enforcement diversion program whereby attendees would be sent to court-ordered treatment instead of jail. Attendees reported receiving legal sanctions for having clean needles obtained at the exchange despite showing proof (i.e., NEP identification card) when being stopped or searched for other reasons:

I had to go to court. I had three of them. They weren't even used. They were brand new. I got a $\$ 195.00$ fine.

These experiences created confusion among NEP attendees who believed that clean needles obtained from the exchange could be possessed, albeit in somewhat limited circumstances: 
They tell you when you first start with the card that it's a one way trip. You got from the exchange to your home and from that point on you're not covered by that card with the needles, only from here to home. It's supposed to be a straight shot and the first time I ever came they explained that to me in full detail that you have a one way trip with them [the police].

Another attendee stated:

They give you a card and I got pulled over and I showed them that card and they said that, that didn't matter at all. All that did was show where I got them from, that it was still paraphernalia and that it was still a misdemeanor.

One attendee described an encounter with a law enforcement officer that felt the NEP itself should be illegal:

I came across a policeman, and he was searching my stuff because I was around someone on house arrest. He was into my stuff. He seen my card from the [NEP]. He was like, "This is illegal. I know why you go there. This is illegal." I'm like, "No, it's not. It's not illegal to go there and get syringes." He's like, "Yes, it is, and I'm going to make sure they close down."

\section{Location Is Everything}

The theme, Location Is Everything, was identified from participant discussions of difficulties related to program utilization stemming from the physical location of the exchanges. One NEP was located very near to the police department, which was acknowledged by all parties interviewed to "absolutely suck." The other NEP was perceived as being too far away to promote regular attendance by attendees in the surrounding community. The Free Clinic NEP has some attendees that come from a rural county that is a two hour drive one way due to the absence of such programs in the local community, and the Health Department NEP expressed an interest in expanding to an adjacent area where the death rate is high but distance precludes attendance by some users. One attendee mentioned driving one hour, one way to obtain injection equipment, which precluded frequent and regular exchange attendance. 
All participants agreed on the need for expanded NEP services at other locations to reach more users. At the time of this writing, the Free Clinic NEP obtained funding to purchase a van for a mobile NEP, and several other NEPs have opened throughout West Virginia with plans for additional programs pending.

\section{Harm Reduction for All}

The theme, Harm Reduction for All, included discussions of how the NEP could positively reduce the spread of disease for all participants (i.e., law enforcement, attendees, and the surrounding community).

\section{Law Enforcement}

Law enforcement support for NEPs was facilitated when the intervention was viewed as an effort to reduce harmful blood borne pathogens that could be contracted by a needle stick occurring during subject searches:

Well myself personally you know l've got a family. You know I don't want to be out here and you know being on the front line dealing with these addicts you know. I don't want to be stuck with a dirty needle. I've been stuck with a dirty needle before and it's not very pleasant the treatment that you have to go through.

\section{Attendees}

NEP attendance was facilitated by attendees' concern with the prevention of abscesses and other blood-borne infections, especially HIV, and most felt that NEPs saved lives:

It's a life changer, really. It's a life changer that you can come up here once or twice a week and get what you need other than going and trying to go and find it [needles] on the street. It's saving lives. (Attendee) 
However, concern over contraction of $\mathrm{HCV}$ was mitigated by the perception that it is an inevitable byproduct of intravenous drug use that all drug users eventually acquire:

It's like, I wear a pair of Levis you know, that's what it's like.

$\mathrm{HCV}$ concerns were also blunted by the fact that many users remain asymptomatic for years:

...You got all these people walking around, well I got Hep and you're looking at them you're like aw they're fine. I mean the long term effects obviously are [bad].

One attendee mentioned that he would feel uncomfortable injecting with an individual who claimed he or she was HCV negative, stating that such an individual might be a member of law enforcement engaged in an undercover sting operation.

For attendees, the fact that these programs are built on the social work principle of meeting people where they currently are in their addiction and keeping them safe that underlies the harm reduction approach, was central to facilitating attendance (Vakharia \& Little, 2017). In particular, the nonjudgmental, welcoming attitude espoused by all program staff was instrumental to encouraging attendance:

Even the woman at the front desk here....she's smiling.

No judgment. Oh yeah that's a big [facilitator], that's your first impression.

Another attendee stated:

The fact that you can come here, and you can get a one-week or a two-week supply. You're not turning people down because of their race, color, or gender. You are welcoming anybody. It's what makes it work. 


\section{Community}

Attendees were particularly concerned with discarded needles and noted the positive benefit of biohazard receptacles that are given to attendees at one NEP for needle disposal:

Well, I think another thing is when everybody gets rid of them, like that actually does come here and get them, they actually throw them away the right way. Don't throw them out the fucking window or anything like that because that's where "junkie" comes from.

Attendees of the other exchange requested that more biohazard containers be made available to reduce dirty discarded needles in the community. Additionally, the Health Department NEP created an environmental response team and engaged in community education. Community members are able to call the response team to come and safely removes needles found in the community.

\section{Not Just A Needle}

The theme, Not Just a Needle, included instances where participants described the importance of other supplies and services in addition to clean needles provided by the exchanges. Attendees appreciated the other comprehensive services provided, such as infectious disease testing and medical care, and linkage to drug treatment was a very important facilitator of program acceptance among law enforcement.

The chiefs of police described the importance of the exchanges as a blood-borne pathogen intervention with a "gateway to recovery" as opposed to a mere harm reduction intervention that encourages continued illicit drug use: 
But then you also have that real fine line. Are you encouraging the continued use of an illegal controlled substance or are you treating it? And I think the panacea is there are two different things. You're trying to intervene on the [blood borne] pathogens. And through that blood borne pathogen intervention you're trying to reach out to change the thought processes of the illicit drug user. And I think that's where people lose the message here that it's not one you know feeding the other. It's one or the other, because that's what you have. The needle exchange is a blood born pathogen intervention not a drug intervention. But they're trying to pull the drug intervention in by getting a hold of them through the medical intervention. (Police Chief)

Future barriers and facilitators to expanding program operations

\section{Surging Volumes: The Storm Before The Calm}

The theme, The Storm Before the Calm, was identified from discussions of unexpected demand for exchange services that created additional barriers to program operations and participation. This demand for the NEPs was greatly underestimated by both programs:

Well we anticipated only having 75 clients in the first year, which was my naïve thinking I guess, our belief that the situation wasn't as bad as it really is. And it was also based on thinking that most of these folks would be people we knew. (Free Clinic NEP Director)

We initially planned for about 500 participants for a year. So September 16 [2016] we expected to be at 500. We were at 500 in about nine weeks. So we immediately knew that we made a small error in judgment. (Health Department NEP Director)

This overwhelming demand, coupled with the previously discussed location and access problems, have led to plans for future expansion by both programs to help calm the storm of attendees. The Free Clinic NEP would like to expand services to more rural counties via a mobile van that was recently purchased; whereas, the Health Department NEP has plans to expand from the current single site to four sites.

Attendees also cited the need for expanded hours of operations:

... what we put in these don't come through no schedule. One things for certain in the dope game, nothing's for certain. ... It's difficult. . Some people can't come on a Wednesday. Some people can't come here all the time. 
However, common to both programs was a shortage of available funding, which significantly threatens the ability to expand:

We can see that we need to expand this, that we are overwhelmed by the volume of patients that we're seeing. ... But we don't have funding to go on with that. And we don't have sustaining funding beyond the first year when you get right down to it. (NEP Director)

\section{Could You Spare Some "Change"?}

The final theme, Could You Spare Some "Change"? was derived from codes assigned to participant discussions of funding barriers related to a federal ban on funding of exchange programs. Although this ban was recently lifted ("changed"), and federal dollars can now be used to fund program operations, both programs have to rely on other sources to fund syringes. The Free Clinic NEP primarily uses money from foundation grants and donors to pay for syringes and other paraphernalia; whereas, the Health Department NEP relied on donations from a local pharmacy of between 13,000 and 15,000 syringes, and another large donor who contributed 60,000 syringes. For both programs, this barrier limits the number of syringes that can be distributed, which can negatively impact the secondary exchange of syringes that occurs when an attendee takes clean needles and distributes them to other PWIDs in the community.

To combat significant funding pressures, both programs cited the ability to leverage resources from nearby universities (Like a Good Neighbor theme), or as one Health Department NEP representative said, "Thank goodness for the students." University affiliated medical and pharmacy professionals also donate their time to each program, which greatly helps offset funding challenges. However, the survival of each program is contingent on the continued support of the surrounding community and 
community champions, particularly law enforcement leadership, given the legal conundrum created by existing policy and laws.

\section{DISCUSSION}

This case study of two needle exchange programs that opened in West Virginia in 2015 reveals common barriers and facilitators that impacted program implementation, operations, and future planning. At the outset, both programs struggled to find existing programs to effectively serve as a model. Although each program had slightly different precipitating events that drove the need for such programs in each community, both observed attendance rates far greater than anticipated, and have faced significant funding challenges that impacts future expansion needs driven by increasing volumes and significant transportation barriers to program attendance. The legal conundrum created by legal distribution of clean needles in the presence of paraphernalia laws rooted in a criminal (i.e., moral) approach to drug use that impacts policing behaviors was a noted barrier to program operations. Despite these challenges, robust community support and the creative leveraging of volunteer resources have facilitated the successful implementation and operation of these programs.

In-depth case studies describing the development and implementation of NEPs in the United States are scant. The literature largely consists of brief descriptions of the first NEPs to open in urban areas of the United States (e.g., Tacoma, San Francisco, New York City, Washington, DC, etc.) in the late 1980 s and early 1990 s in response to the HIV crisis (Anderson, 1991; Hagan, Des Jarlais, Purchase, Reid, \& Friedman, 1991; Kaplan \& O'Keefe, 1993; Sherman \& Purchase, 2001; Somlai, Kelly, Otto-Salaj, \& Nelson, 1999; Vlahov et al., 1994; Watters, Estilo, Clark, \& Lorvick, 1994), Only two 
studies (Sherman \& Purchase, 2001; Somlai et al., 1999) were located that specifically described the use of qualitative research methodologies to obtain an in-depth program (i.e., case) understanding. However, these differences notwithstanding, there were a number of similarities with regard to barriers and facilitators experienced by these urban programs and the two West Virginia exchanges that comprise this case study.

First, as was experienced by both West Virginia programs, there was no readily available model exchange in the United States to guide program design and implementation (One Size Doesn't Fit All theme). Rather, many urban programs used the first NEPs that opened in Amsterdam as a guide to program development (Anderson, 1991; Hagan et al., 1991; Kaplan \& O'Keefe, 1993; Sherman \& Purchase, 2001). Other programs engaged the surrounding community during program design and implementation (Kaplan \& O'Keefe, 1993; Somlai et al., 1999). For example, the Lifepoint NEP case study described the use of qualitative ethnographic methods and systemic community analysis in the design and implementation of a NEP in Milwaukee, Wisconsin (Somlai et al., 1999), which is similar to the community advisory board and key informants used to guide the development of the Free Clinic NEP. Program planners also engaged community leaders and law enforcement in the design and implementation of a NEP in New Haven, Connecticut (Kaplan \& O'Keefe, 1993).

Community support was cited as critical to program implementation and continuing operations by a few studies (Kaplan \& O'Keefe, 1993; Sherman \& Purchase, 2001; Somlai et al., 1999), especially support from community leaders such as the chief of police, the mayor, and the health department director (Like a Good Neighbor theme). In particular, the initial support of the health department was cited several times 
throughout the Point Defiance NEP case study in Tacoma, Washington (Sherman \& Purchase, 2001) as a prominent factor in the successful implementation and ongoing operation of the exchange, and has been critical to the success of the Health Department NEP in this case study. Although both programs in our case study enjoyed broad support from the chiefs of police, support among rank-and-file officers was somewhat divided, which likely contributed to the negative experiences with law enforcement shared by the attendees interviewed. To promote police acceptance of the NEP, program leaders of the LifePoint NEP involved law enforcement in the planning process, as did both of the West Virginia programs in this case study. Although no attendees reported police harassment after the LifePoint NEP implementation, the overall attitude of law enforcement with regard to the program remained largely neutral (Somlai et al., 1999).

Most urban programs have also described difficulties stemming from the existence of paraphernalia laws and associated policing behavior (The Legal Conundrum theme) (Hagan et al., 1991; Kaplan \& O'Keefe, 1993; Vlahov et al., 1994). The Point Defiance NEP did not experience many operational barriers, at least initially, primarily because the chief of police made a conscious decision to not arrest the founder for violation of state paraphernalia laws. Additionally, the state Supreme Court later ruled in favor of the exchange finding that public health interests superseded existing drug paraphernalia laws (Sherman \& Purchase, 2001). However, it is unknown whether or not rank-and-file law enforcement officers also chose to ignore paraphernalia laws in their dealings with PWIDs, which was a noted barrier reported by attendees in our setting. 
Funding was a major barrier cited by both West Virginia programs that impacted both current operations and needed expansion plans (Could You Spare Some "Change" theme). A notable exception to this case is the Point Defiance NEP in Tacoma, Washington where community support minimized financial barriers. Funding from private citizens, in addition to city and county sources, were cited as contributing factors to a program budget that continued to grow (Sherman \& Purchase, 2001). Similarly, the LifePoint NEP case did not report funding difficulties (Somlai et al., 1999). However, funding difficulties have been reported more recently by NEPs in the United States regardless of geographical location, although more rural programs reported funding difficulties in comparison to their urban and suburban counterparts (Des Jarlais et al., 2015).

NEP funding difficulties were exacerbated by exponential growth that necessitated the need for geographic expansion (The Storm Before the Calm and Location Is Everything themes). This expansion involved delivery of needles to attendees who did not or could not attend the original fixed site location, which is similar to the expansion plans of the Free Clinic NEP (i.e., van delivery of supplies to an adjacent county) in this case study, and another study in which NEP attendees listed inconvenient location/hours as a barrier to exchange participation (Rich, Strong, Towe, \& McKenzie, 1999). Other urban programs have also cited the benefits from using a mobile versus fixed site program model to reach more PWIDs (Kaplan \& O'Keefe, 1993; Somlai et al., 1999).

Although NEPs provide all supplies needed for the injection process due to the belief that HCV can be present in more than the needle, a very recent study has cast 
doubt on the utility of this approach. Experiments were designed to replicate drug sharing practices, but no HCV could be recovered from the cookers. Furthermore, HCV was only recovered in $15.4 \%$ of detachable filters and $1.4 \%$ of fixed filters (Heimer et al., 2017). Although additional research is necessary to replicate this preliminary study, the authors conclude that NEPs faced with financial difficulties may consider focusing resources on the syringes themselves versus the additional injection equipment.

NEP funding has historically been inextricably intertwined with paraphernalia laws in the United States (Golding, 2017). In a recent analysis of the comprehensiveness of state laws to prevent HCV (Campbell et al., 2017), West Virginia was one of 18 states with the least comprehensive laws. These states had no authorization of statewide syringe exchange, no laws decriminalizing possession and distribution of syringes, and no laws explicitly allowing the retail sales of syringes without a prescription. The absence of explicit direction from the state may have contributed to differing practices among law enforcement officers related to whether or not attendees should be legally sanctioned if found to be in possession of syringes, either dirty or clean.

Law enforcement attitudes toward PWIDs may also impact the approach to drug paraphernalia. Although the law enforcement officials participating in this case study were generally supportive of NEPs, many acknowledged that their fellow officers may share divergent views (i.e., in favor of a criminal approach to drug paraphernalia), and the experiences of NEP attendees highlighted this diversity of opinions. A qualitative study of the NEP barriers to implementation conducted in China observed similar results. As was the case in our study, law-enforcement officials in China tended to view 
NEP as a temporary measure in place of legal sanctions, and some viewed these programs as an explicit encouragement of drug use (Koo et al., 2015).

Recent evidence has suggested a link between syringe sharing and police confiscation of needles, both clean and used. A cross-sectional survey of IDUs in Tijuana and Ciudad Juarez, Mexico observed increased odds of receptive syringe sharing from arrests for possessing both clean needles (aOR 2.05, 95\% Cl, 1.26, 3.35) and used needles (aOR 2.87, 95\% Cl, 1.76, 4.69) (Pollini et al., 2008). These findings are particularly concerning given the fact that syringe sharing is associated with a $94 \%$ increased risk of acquiring HCV (Pouget, Hagan, \& Des Jarlais, 2012). However, the impact of these paraphernalia laws and policing behaviors on the ability of PWIDs to both bring clean needles back for exchange and have clean needles available for use during injection in a rural area is currently unknown and warrants further inquiry.

\section{Limitations}

Some study limitations should be noted. It is possible that responses may have been impacted by social desirability bias given the sensitive topic. However, no names or demographic information were collected or reported to help minimize the impact of this bias and increase the validity of the measurement. Additionally, the experiences of other rural programs in both West Virginia and other locales may be somewhat different than the experiences described in this case study given that the small sample size may have precluded the ability to achieve saturation of opinions, views, and themes. 


\section{Reflexivity}

The primary author's social work background and experience with individuals suffering from addiction could have affected the interpretation of the results and led to a positive evaluation of many aspects of the program.

\section{CONCLUSIONS}

Due to overwhelming need, PWIDs are often in the dark regarding the precise outcomes that will be experienced during their next injection. Will it be the sought-after high or a disappointing low? Will it be a fatal overdose or a 'near-miss'? Similarly, the two West Virginia needle exchange programs described in this case study have been forced to take a shot in the dark and open NEPs in the absence of model programs, adequate funding, and other resources, due to overwhelming need in their small communities. Despite these challenges, such programs have enjoyed robust community support, and have creatively navigated unexpected problems and challenges to effectively implement needle exchange programs in their rural communities. However, surging attendee volumes amidst ongoing funding challenges coupled with location and transportation barriers make the future trajectory of such programs difficult to predict. Additionally, the efficacy of these programs in preventing transmission of blood borne viruses may be compromised from the legal conundrums created by paraphernalia laws and policing behaviors that may promote needle sharing that is the primary risk for acquiring HCV among injection drug users. Future studies should investigate these potential barriers to using clean needles in rural injection drug

users. Studies elucidating factors contributing to the funding disparity experienced by rural NEPs in comparison to their urban and suburban counterparts are also needed. 


\section{REFERENCES}

Anderson, W. (1991). The New York Needle Trial: the politics of public health in the age of AIDS. Am J Public Health, 81(11), 1506-1517.

Atchan, M., Davis, D., \& Foureur, M. (2016). A methodological review of qualitative case study methodology in midwifery research. J Adv Nurs, 72(10), 2259-2271. doi:10.1111/jan.12946

Beheshti, A., Lucas, L., Dunz, T., Haydash, M., Chiodi, H., Edmiston, B., . . Horzempa, J. (2015). An Evaluation of Naloxone Use for Opioid Overdoses in West Virginia: A Literature Review. Am Med J, 6(1), 9-13. doi:10.3844/amjsp.2015.9.13

Brinkmann, S., Kvale S. (2015). Interviews: Learning the Craft of Qualitative Research Interviewing (3rd ed.). Thousand Oaks, CA: Sage.

Campbell, C. A., Canary, L., Smith, N., Teshale, E., Ryerson, A. B., \& Ward, J. W. (2017). State HCV Incidence and Policies Related to HCV Preventive and Treatment Services for Persons Who Inject Drugs United States, 2015-2016. MMWR Morb Mortal Wkly Rep, 66(18), 465-469.

doi:10.15585/mmwr.mm6618a2

CDC. (2017). Viral Hepatitis Surveillance - United States, 2015. Retrieved $9^{\text {th }}$ September 2017 from Viral Hepatitis, Surveillance for Viral Hepatitis-United States, 2015 website: https://www.cdc.gov/hepatitis/statistics/2015surveillance/commentary.htm

Cooke, G. S., Lemoine, M., Thursz, M., Gore, C., Swan, T., Kamarulzaman, A., . . Ford, N. (2013). Viral hepatitis and the Global Burden of Disease: a need to regroup. J Viral Hepat, 20(9), 600-601. doi:10.1111/jvh.12123

Creswell, J. W. (2013). Qualitative Inquiry and Research Design: Choosing Among Five Approaches (3rd ed.). Los Angeles, CA: Sage.

Csete, J., Kamarulzaman, A., Kazatchkine, M., Altice, F., Balicki, M., Buxton, J., . . B Beyrer, C. (2016). Public Health and International Drug Policy: Report of the Johns Hopkins - Lancet Commission on Drug Policy and Health. Lancet (London, England), 387(10026), 1427-1480. doi:10.1016/S01406736(16)00619-X

Davis, S. M., Daily, S., Kristjansson, A. L., Kelley, G. A., Zullig, K., Baus, A., . . Fisher, M. (2017). Needle exchange programs for the prevention of hepatitis $C$ virus infection in people who inject drugs: a systematic review with meta-analysis. Harm Reduct J, 14(1), 25. doi:10.1186/s12954-017-0156-z

Denzin, N. K., Lincoln, Y.S. (2011). Introduction: The Discipline and Practice of Qualitative Research (4th ed.). Thousand Oaks, CA: Sage.

Des Jarlais, D. C. (2017). Harm reduction in the USA: the research perspective and an archive to David Purchase. Harm Reduct J, 14(1), 51. doi:10.1186/s12954-017-0178-6

Des Jarlais, D. C., Nugent, A., Solberg, A., Feelemyer, J., Mermin, J., \& Holtzman, D. (2015). Syringe Service Programs for Persons Who Inject Drugs in Urban, Suburban, and Rural Areas - United States, 2013. MMWR Morb Mortal Wkly Rep, 64(48), 1337-1341. doi:10.15585/mmwr.mm6448a3 
Estes, C., Abdel-Kareem, M., Abdel-Razek, W., Abdel-Sameea, E., Abuzeid, M., Gomaa, A., ... Waked, I. (2015). Economic burden of hepatitis $C$ in Egypt: the future impact of highly effective therapies. Aliment Pharmacol Ther, 42(6), 696-706. doi:10.1111/apt.13316

Golding, N. J. (2017). The Needle and the Damage Done: Indiana's Response to the 2015 HIV Epidemic and the Need to Change State and Federal Policies regarding Needle Exchanges and Intravenous Drug Users. Indiana Health Law Review, 14(2), 173-220.

Hagan, H., Des Jarlais, D. C., Purchase, D., Reid, T., \& Friedman, S. R. (1991). The Tacoma Syringe Exchange. J Addict Dis, 10(4), 81-88. doi:10.1300/J069v10n04_06

Harry, B. (2005). Mapping the process: an exemplar of process and challenge in grounded theory analysis. Educational Researcher, 34(2), 3-13.

Havens, J. R., Lofwall, M. R., Frost, S. D. W., Oser, C. B., Leukefeld, C. G., \& Crosby, R. A. (2013). Individual and Network Factors Associated With Prevalent Hepatitis C Infection Among Rural Appalachian Injection Drug Users. American Journal of Public Health, 103(1), e44-e52. doi:10.2105/ajph.2012.300874

Kaplan, E. H., \& O'Keefe, E. (1993). Let the Needles Do the Talking! Evaluating the New Haven Needle Exchange. Interfaces, 23(1), 7-26.

Keeshin, S. W., \& Feinberg, J. (2016). Endocarditis as a Marker for New Epidemics of Injection Drug Use. Am J Med Sci, 352(6), 609-614. doi:10.1016/j.amjms.2016.10.002

Koo, F. K., Chen, X., Chow, E. P., Jing, J., Zheng, J., Zhao, J., \& Zhang, L. (2015). Barriers and Potential Improvements for Needle and Syringe Exchange Programs (NSPs) in China: A Qualitative Study from Perspectives of Both Health and Public Security Sectors. PLOS ONE, 10(6), e0130654.

doi:10.1371/journal.pone.0130654

MacArthur, G. J., van Velzen, E., Palmateer, N., Kimber, J., Pharris, A., Hope, V., . . Hutchinson, S. J. (2014). Interventions to prevent HIV and Hepatitis $C$ in people who inject drugs: A review of reviews to assess evidence of effectiveness. International Journal of Drug Policy, 25(1), 34-52.

doi:10.1016/j.drugpo.2013.07.001

Macworld. (2016). Record voice memos on the ipod, iPhone. Retrieved on $1^{\text {st }}$ October 2017 from http://www.macworld.com/article/1152402/entertainment/voice-memos.html

Miles, M. B. (1994). Qualitative Data Analysis: A Sourcebook of New Methods (2nd ed.). Thousand Oaks, CA: Sage.

North American Syringe Exchange Network. (2018). Directory of Syringe Exchange Programs. Retrieved $2^{\text {nd }}$ January 2018 from https://nasen.org/directory/wv/s

NVIVO. (2016). NVIVO Software. Retrieved $1^{\text {st }}$ October 2017 from http://www.qsrinternational.com/product

Page, K., Morris, M. D., Hahn, J. A., Maher, L., \& Prins, M. (2013). Injection Drug Use and Hepatitis C Virus Infection in Young Adult Injectors: Using Evidence to Inform Comprehensive Prevention. Clinical Infectious Diseases, 57(suppl_2), S32-S38. doi:10.1093/cid/cit300

Petruzziello, A., Marigliano, S., Loquercio, G., Cozzolino, A., \& Cacciapuoti, C. (2016). Global epidemiology of hepatitis $C$ virus infection: An up-date of the distribution and circulation of hepatitis $C$ virus genotypes. World J Gastroenterol, 22(34), 7824-7840. doi:10.3748/wjg.v22.i34.7824 
Pollini, R. A., Brouwer, K. C., Lozada, R. M., Ramos, R., Cruz, M. F., Magis-Rodriguez, C., ... Strathdee, S. A. (2008). Syringe possession arrests are associated with receptive syringe sharing in two Mexico-US border cities. Addiction, 103(1), 101-108. doi:10.1111/j.1360-0443.2007.02051.x

Pouget, E. R., Hagan, H., \& Des Jarlais, D. C. (2012). Meta-analysis of hepatitis C seroconversion in relation to shared syringes and drug preparation equipment. Addiction, 107(6), 1057-1065. doi:10.1111/j.1360-0443.2011.03765.x

Rich, J. D., Strong, L., Towe, C. W., \& McKenzie, M. (1999). Obstacles to needle exchange participation in Rhode Island. J Acquir Immune Defic Syndr, 21(5), 396-400.

Saldana, J. (2016). The Coding Manual for Qualitative Researchers (3rd ed.). Thousand Oaks, CA: Sage.

Sandelowski, M., Barroso, J. (2007). Handbook for Synthesizing Qualitative Research. New York, NY: Springer.

Sherman, S. G., \& Purchase, D. (2001). Point Defiance: a case study of the United States' first public needle exchange in Tacoma, Washington. International Journal of Drug Policy, 12(1), 45-57. doi:10.1016/s0955-3959(00)00074-8

Smith, B. D., Morgan, R. L., Beckett, G. A., Falck-Ytter, Y., Holtzman, D., Teo, C. G., . . W Ward, J. W. (2012). Recommendations for the identification of chronic hepatitis $C$ virus infection among persons born during 1945-1965. MMWR Recomm Rep, 61(Rr-4), 1-32.

Somlai, A. M., Kelly, J. A., Otto-Salaj, L., \& Nelson, D. (1999). "Lifepoint": a case study in using social science community identification data to guide the implementation of a needle exchange program. AIDS Educ Prev, 11(3), 187-202.

Stake, R. (1995). The Art of Case Study Research. Tousand Oaks, CA: Sage.

Stancliff, S., Phillips, B. W., Maghsoudi, N., \& Joseph, H. (2015). Harm Reduction: Front Line Public Health. J Addict Dis, 34(2-3), 206-219. doi:10.1080/10550887.2015.1059651

Stepanova, M., \& Younossi, Z. M. (2017). Economic Burden of Hepatitis C Infection. Clinics in Liver Disease, 21(3), 579-594. doi:10.1016/j.cld.2017.03.012

Strathdee, S. A., \& Beyrer, C. (2015). Threading the Needle - How to Stop the HIV Outbreak in Rural Indiana. New England Journal of Medicine, 373(5), 397-399. doi:10.1056/nejmp1507252

Suryaprasad, A. G., White, J. Z., Xu, F., Eichler, B.-A., Hamilton, J., Patel, A., . . Holmberg, S. D. (2014). Emerging Epidemic of Hepatitis C Virus Infections Among Young Nonurban Persons Who Inject Drugs in the United States, 2006-2012. Clinical Infectious Diseases, 59(10), 1411-1419. doi:10.1093/cid/ciu643

TASCAM. (2016). TASCAM DR-05. Retrieved $1^{\text {st }}$ October 2017 from http://tascam-ca.com/product/dr$05 /$

Tempalski, B., Flom, P. L., Friedman, S. R., Des Jarlais, D. C., Friedman, J. J., McKnight, C., \& Friedman, R. (2007). Social and Political Factors Predicting the Presence of Syringe Exchange Programs in 96 US Metropolitan Areas. American Journal of Public Health, 97(3), 437-447. doi:10.2105/ajph.2005.065961

Thrift, A. P., El-Serag, H. B., \& Kanwal, F. (2016). Global epidemiology and burden of HCV infection and HCV-related disease. Nature Reviews Gastroenterology \& Hepatology, 14(2), 122-132.

doi:10.1038/nrgastro.2016.176 
Tong, A., Sainsbury, P., \& Craig, J. (2007). Consolidated criteria for reporting qualitative research (COREQ): a 32-item checklist for interviews and focus groups. Int J Qual Health Care, 19(6), 349-357. doi:10.1093/intqhc/mzm042

U.S. Census Bureau. (2017). Quick Facts: West Virginia. Retrieved $8^{\text {th }}$ October 2017 from https://www.census.gov/quickfacts/fact/table/WV/PST045216

Vakharia, S. P., \& Little, J. (2017). Starting Where the Client Is: Harm Reduction Guidelines for Clinical Social Work Practice. Clinical Social Work Journal, 45(1), 65-76. doi:10.1007/s10615-016-0584-3

Vlahov, D., Des Jarlais, D. C., Goosby, E., Hollinger, P. C., Lurie, P. G., Shriver, M. D., \& Strathdee, S. A. (2001). Needle Exchange Programs for the Prevention of Human Immunodeficiency Virus Infection: Epidemiology and Policy. American Journal of Epidemiology, 154(12), S70-S77. doi:10.1093/aje/154.12.s70

Vlahov, D., Ryan, C., Solomon, L., Cohn, S., Holt, M. R., \& Akhter, M. N. (1994). A pilot syringe exchange program in Washington, DC. Am J Public Health, 84(2), 303-304.

Ward, J. W. (2013). The hidden epidemic of hepatitis C virus infection in the United States: occult transmission and burden of disease. Top Antivir Med, 21(1), 15-19.

Watters, J. K., Estilo, M. J., Clark, G. L., \& Lorvick, J. (1994). Syringe and needle exchange as HIV/AIDS prevention for injection drug users. JAMA, 271(2), 115-120.

WV DHHR. (2017). Incidence Rate of Acute Hepatitis C (per 100,000 populations) by County of Residence, West Virginia, Year of Onset 2007-2015. Retrieved $1^{\text {st }}$ October 2017 from West Virginia Department of Health and Human Resources, Office of Epidemiology and Prevention Services, Hepatitis C website: https://dhhr.wv.gov/oeps/disease/viral-hepatitis/Pages/HepC.aspx

Yin, R. K. (2016). Case Study Research: Design and Methods (5th. ed.). Thousand Oaks, CA: Sage.

Zare, F., Fattahi, M. R., Sepehrimanesh, M., \& Safarpour, A. R. (2016). Economic Burden of Hepatitis C Virus Infection in Different Stages of Disease: A Report From Southern Iran. Hepatitis Monthly, 16(4), e32654. doi:10.5812/hepatmon.32654

Zibbell, J. E., Iqbal, K., Patel, R. C., Suryaprasad, A., Sanders, K. J., Moore-Moravian, L., . . Prevention. (2015). Increases in hepatitis $C$ virus infection related to injection drug use among persons aged $</=30$ years - Kentucky, Tennessee, Virginia, and West Virginia, 2006-2012. MMWR Morb Mortal Wkly Rep, 64(17), 453-458.

Zibbell, J. E., Asher, A. K., Patel, R. C., Kupronis, B., Iqbal, K., Ward, J. W., \& Holtzman, D. (2018). Increases in Acute Hepatitis C Virus Infection Related to a Growing Opioid Epidemic and Associated Injection Drug Use, United States, 2004 to 2014. Am J Public Health, 108(2), 175-181. doi:10.2105/ajph.2017.304132 


\subsection{Study 3 Manuscript}

\section{Front Sheet}

Title: Barriers to Using Clean Needles Encountered By Rural Appalachian People Who Inject Drugs: Implications for Needle Exchange

Authors: Stephen M. Davis (smdavis@hsc.wvu.edu), ${ }^{1}$ Alfgeir L. Kristjansson

(alkristjansson@hsc.wvu.edu), ${ }^{2}$ Danielle Davidov (ddavidov@hsc.wvu.edu), ${ }^{12}$ Keith

Zullig (kzullig@hsc.wvu.edu),,2 Adam Baus (abaus@hsc.wvu .edu),,2 Melanie Fisher (mfisher@hsc.wvu.edu) $)^{3}$

Author Affiliations: 'Department of Emergency Medicine, West Virginia University, PO Box 9149, Morgantown, WV 26506, United States

${ }^{2}$ Department of Social and Behavioral Sciences, West Virginia University, PO Box 9190, Morgantown, WV 26506, United States ${ }^{3}$ Department of Medicine, Section of Infectious Diseases, West Virginia University, PO Box 9163, Morgantown, WV 26506, United States

Running Head: Barriers to Using Clean Needles and Needle Exchange Word Count (excluding abstract, references, tables, and figures): 3,508

Address for Correspondence: Stephen M. Davis, Adjunct Professor, School of Medicine, Department of Emergency Medicine, Robert C. Byrd Health Sciences Center, West Virginia University, PO Box 9149, Morgantown, WV, United States, 26506-9149, Office Phone: 304-293-1326, Fax: 304-293-6702, E-mail: smdavis@hsc.wvu.edu

Declaration of Competing Interest: None 
Funding: The West Virginia University Department of Emergency Medicine provided funding for the incentives given to survey participants. However, the Department had no direct role in drafting, interpretation, and analysis of results.

\section{ABSTRACT}

Background and Aims. Using a new (clean) needle for every injection can reduce the spread of infectious disease among people who inject drugs (PWID). However, barriers to using clean needles are not well understood, and no previous study has examined these barriers among PWIDs residing in the rural Appalachian part of the United States, an area currently in the midst of a heroin epidemic. Therefore, our primary aim was to explore self-reported barriers to using a clean needle by PWIDs attending a needle exchange program (NEP). Design and Setting. We conducted a cross-sectional survey of PWIDs attending two NEPs in rural West Virginia located in the heart of Central Appalachia. Measurement. A convenience sample of PWIDs $(n=100)$ completed the Barriers to Using New Needles Questionnaire. Findings. The median number of barriers reported was 5 (range $0-19$ ). Older age and longer injection history were significantly associated $(p<0.05)$ with reporting $>8$ barriers. Fear of arrest by police (72\% of PWIDs "agreed" or "strongly agreed") and difficulty with purchasing needles from a pharmacy (64\% "agreed" or "strongly agreed") were the most frequently cited barriers.

Conclusions. Congruent with previous findings from urban heroin users, in rural West Virginia, the ability of people who inject drugs to use a clean needle obtained from a needle exchange for every injection may be compromised by fear of arrest. In addition, pharmacy sales of clean needles to people who inject drugs may be blunted by an 
absence of explicit laws mandating nonprescription sales. Future studies should explore interventions that align the public health goals of needle exchange programs with the occupational safety of law enforcement and health outreach goals of pharmacists.

Keywords: needle exchange programs; hepatitis C virus; people who inject drugs; paraphernalia laws; barriers to using clean needles; policing behaviors

\section{INTRODUCTION}

The United States is currently in the midst of a hepatitis C virus (HCV) epidemic that has been catalyzed by injection drug use in rural areas. Cases of acute HCV infection increased $98 \%$ between 2010 and 2015 with consistent annual increases observed during this time period (1). Approximately 60 to $70 \%$ of HCV cases in the United States occur in people who inject drugs (PWID) (2, 3). A disproportionate number of new HCV cases occur in young, white PWIDs residing in the rural, Central Appalachian region of the United States, which includes the states of Kentucky, Tennessee, Virginia, and West Virginia $(1,2,4-6)$. This region observed a $364 \%$ increase in new HCV cases between 2006 and 2012, (5) and three of the four states that comprise this region (West Virginia, Kentucky, and Tennessee) had the highest rate of new HCV infections in the United States (1). This exponential increase in HCV cases has been associated with prescription opioid misuse that progresses to injection of heroin (7-10).

Among PWIDs, the risk of HCV seroconversion increases $94 \%$ when syringes are shared during injection (11). In an effort to minimize the reuse of needles, needle exchange programs (NEP) have been implemented as a mechanism for exchanging 
used needles for clean (new) needles $(9,12)$. Although the ability to access clean needles is one important step in fighting the HCV epidemic, there are potential barriers to practicing risk reduction strategies (e.g., not sharing syringes) that may reduce the efficacy of NEPs. Barriers to using a clean needle for every injection can occur at the individual (e.g., being in drug withdrawal), interpersonal (e.g., peer influences), and societal (e.g., confiscation of needles by police) levels of influence (13), although there is a dearth of studies that empirically measure these barriers. For example, after a thorough review of the extant literature, only one study was located. In this study heroin injectors in Denver, Colorado in the United States were recruited and administered a structured interview that explored barriers associated with risk reduction practices (13). Being in drug withdrawal and fear of being arrested with needles were the two most frequently reported barriers to using new needles in this urban sample. However, the barriers to using clean needles that may exist within the rural parts of the United States that are driving the recent HCV epidemic are currently unknown (14). Therefore, the aim of this exploratory study was to identify self-reported barriers to using a clean needle among PWIDs attending NEPs in the state of West Virginia, which is located in the heart of Central Appalachia in the United States. Improved understanding of these barriers may have implications for the efficacy of needle exchange for the prevention of HCV. Based on the Denver study (13), we hypothesized that fear of arrest and drug withdrawal would be the most frequently reported barriers to using a clean needle. 


\section{METHODS}

\section{Design}

We conducted a cross-sectional survey of PWIDs attending the first two NEPs to open in West Virginia, the Cabell-Huntington Health Department Syringe Exchange (15) and the Mylan Puskar Health Right LIGHT (Living in Good Health Together) Program (16).

\section{Measures}

We adapted the Barriers to Using New Needles Questionnaire (Cronbach's alpha $=0.84)$ that was developed by Phillips et al. (13) and administered to a convenience sample of urban heroin users in Denver, Colorado to measure clean needle use barriers in our setting. This questionnaire consists of 20 possible barriers to using a clean needle gleaned from clinical work and qualitative interviews. Each participant indicates the extent to which s/he agrees with each item (barrier) according to a 5-point Likert scale ranging from "strongly disagree" to "strongly agree".

Prior to survey administration, we purposely selected and interviewed up to 5 attendees at each NEP using methods similar to the technique of respondent debriefings (17). Specifically, attendees were queried about survey comprehension and completeness (18). Guiding questions were based on: 1) whether or not items were understandable; 2) whether or not response choices were adequate; 3) whether or not items were written in a manner that elicited only one response; and, 4) whether or not items were complete. Item modifications were made as needed to maximize their applicability to the rural setting and avoid poorly defined words or terms that were not 
universally understood (19). To further enhance reliability and increase validity, the directors of both NEPs also reviewed these items.

After item modifications, basic demographic questions querying age, gender, race/ethnicity, education level, employment status, housing status, HCV status, and injection history (e.g., frequency of injecting, years of injecting, etc.) were included in the final questionnaire (see Appendix). To minimize potential bias stemming from participant and item nonresponse, the final survey was designed and pilot tested to take no more than 3-5 minutes to complete $(18,20)$. Importantly, to minimize social desirability bias given the sensitive nature of the questions, the survey was anonymous (19).

\section{Participants and Procedure}

Based on consultation with the NEP program directors, a purposeful sample of 100 attendees was planned. This sample size was selected based on the following considerations: 1) Director's assessment of maximum number of accessible participants; 2) the unstable and transient nature of the target population; and, 3) the minimum number needed to allow calculation of statistical differences in reported barriers by demographics and injection history. To promote the number of responses, participants were personally invited by study staff to take the survey while attending the exchange (20). The only requirements for participation were current exchange attendance and being at least 18 years of age. Participants were given a $\$ 10$ gift card upon completion of the survey. The West Virginia University Institutional Review Board approved the study and waived the requirement for written informed consent given that 
the only document linking participants to the sensitive and potentially stigmatizing survey would be the consent form.

\section{Statistical analysis}

All survey data were entered into JMP® 13.0 Pro (21). Item reliability was assessed using Cronbach's $\alpha$. The median was imputed in cases where respondents gave a range of values instead of a single number (i.e., 5-10 injections $=7.5$ injections; $47 \%$ of cases) and in the $8 \%$ of cases with missing injection history. Frequencies and descriptive statistics were calculated for all measured variables. The extent to which demographic characteristics differed between respondents at each NEP was assessed using chi-square (or Fisher's exact test when more than $20 \%$ of expected cell counts were $<5$ ) for nominal or ordinal variables, and the t-test (or Wilcoxon test in the case of non-normally distributed outcomes) for continuous variables. Normality of outcomes was assessed via the Shapiro-Wilk W test. Continuous variables that significantly departed from normality were recoded into an ordinal variable based on distribution quartiles and a goal of creating category cell sizes that met the chi-square test requirement of no more than $20 \%$ of cells with expected counts $<5$.

\section{Barriers to using clean needles}

The 5-point Likert response indicating agreement with each barrier was collapsed into a dichotomous "agree" (responses of "agree", "strongly agree") and "disagree" (responses of "strongly disagree", "disagree", "neutral") variable for analysis to allow comparisons with a previous cohort of Denver heroin injectors that completed the Barriers to Using New Needles Questionnaire (13). Contingency table analysis was 
used to explore associations between nominal and ordinal demographic and injection history factors and 1$)$ the number of barriers reported $(<4,4-8,>8)$; and, 2$)$ agreement with the individual barriers to clean needle use that were endorsed by more than $50 \%$ of respondents. Analysis of variance (ANOVA) was used to examine associations between age and reported barriers. An alpha of 0.05 was selected as the threshold for statistical significance.

\section{RESULTS}

\section{Participants}

To test the comprehension and completeness of the survey, two focus groups with 8 PWIDs (4 males, 4 females) drawn from attendees at both the Health Department NEP $(n=5)$ and the LIGHT Program $(n=3)$ were conducted. All respondents felt that the Barriers to Using New Needles Questionnaire items were complete (i.e., no potential barriers were missed) and written in a manner that only elicited one response.

Participants also felt that response choices (i.e., the 5-point Likert scale) were adequate and that most items were understandable with the exception of item 8 that discussed the potential barrier of using a new needle while injecting in a shooting gallery. Several participants were unfamiliar with the term "shooting gallery". Therefore, we added a clarifying definition and a few terms (i.e., "dope den", "joy popping") taken directly from the transcripts of the focus groups that reflect the concept of a shooting gallery in our setting. Otherwise, no other changes were made to the questionnaire (see table 2 for specifics). 
Overall, 100 PWIDs (Health Department, $n=74$; LIGHT Program, $n=26$ ) were approached and agreed to take the modified questionnaire (Cronbach's $\alpha=0.88$ ). Respondents were generally young (i.e., $<40$ yrs.; range = 18-63 yrs.) and NonHispanic with slightly more males than females completing the survey. Most respondents $(78 \%)$ reported no education beyond high school, and $2 / 3^{\text {rds }}(66 \%)$ were either unemployed or unable to work. A substantial proportion of respondents (40\%) reported that they were currently homeless and $1 / 3^{\text {rd }}$ were HCV positive. Respondents reported injecting between 1 and 18 times a day, 2 to 150 times a week, for 1 month to 35 years. There were no statistically significant differences between NEP sites in any of the demographic variables measured (see Table 1).

\section{Drug of choice}

Heroin was the most frequently cited drug of choice by respondents $(n=67$, $71.27 \%)$. Other drugs of choice included methamphetamine $(n=16,17.02 \%)$, buprenorphine ( $n=11,11.70 \%)$, cocaine $(n=2,7.45 \%)$, fentanyl $(n=1,2.13 \%)$, and amphetamines $(n=1,1.06 \%)$.

\section{Barriers to using clean needles}

\section{Number of barriers reported}

The median number of barriers to using clean needles among all participants was 5 (range $0-19$ ). PWIDs who reported $>8$ barriers were significantly older than those reporting $<4$ barriers $(41.87 \mathrm{yrs}$. vs. $35.68 \mathrm{yrs}$, respectively; $p=0.02)$. PWIDs who had been injecting for at least 8 years were significantly more likely to report $>8$ barriers to clean needle use compared to those injecting for $<3$ years $(41.67 \%$ versus 
$12.50 \%$, respectively; $p=0.048$ ). There were no other significant differences (all $p>$ 0.05 ) in the number of barriers reported between sites, demographic categories, or by frequency of injection.

\section{Barriers reported by more than half of respondents}

Among all 20 barriers, three items were endorsed by at least $50 \%$ of respondents (see Table 2). Two of these barriers related to obtaining clean needles at pharmacies (items 11,13), and one reflected fear of arrest (item 20). Fear of arrest was the most frequently cited barrier overall with $45 \%$ of respondents strongly agreeing and an additional $27 \%$ agreeing that it was a barrier to using clean needles. Agreement with these three items did not differ significantly (all $p>0.05$ ) by age, gender, race, ethnicity, education, employment status, housing status, HCV status, or injection history.

\section{DISCUSSION}

We conducted the first study to empirically assess barriers to using clean needles by people who inject drugs and live in the Appalachian region of the United States that is currently in the midst of an opioid and hepatitis $C$ epidemic. As hypothesized, similar to urban, Denver, Colorado, fear of arrest by law enforcement officials was the most frequently reported barrier to using clean needles in our population. However, we did not find being in withdrawal to be a significant barrier to clean needle use as we expected. Rather, difficulty obtaining clean needles from pharmacies was the second most frequently cited barrier. To our knowledge, this is the largest empirical study examining barriers to using clean needles as reported by the hard-to-reach PWID population currently attending a needle exchange program. These 
findings may have implications for the efficacy of needle exchange programs for the prevention of hepatitis $\mathrm{C}$ and other infectious diseases among PWIDs.

Previous studies examining barriers to practicing risk reduction strategies by PWIDs attending needle exchange programs have employed qualitative methods and focused on harm reduction strategies other than needle exchange (e.g., skin cleaning and test shots). A seminal study by Williams (22) with 15 injection drug using women employed qualitative methods and examined barriers to "safe drug use", but did not examine barriers to specific harm reduction strategies (i.e. using clean needles), although access to clean needles was cited as a barrier to safe drug use. Latkin et al. (23) examined potential barriers to sharing needles before first cleaning them with bleach in 413 PWIDs residing in Baltimore, Maryland in the United States, and Gleghorn and Corby (24) conducted 17 focus groups in eight US cities with 154 PWIDs also looking at willingness to adopt guidelines for bleach disinfection of needles and syringes. However, there is no evidence that cleaning needles with bleach is an effective strategy against the prevention of HCV in PWIDs (25), and the US Centers for Disease Control only recommends bleaching needles when clean, sterile needles are unavailable (26). Therefore, in the present study, we were only interested in examining barriers to using a new needle. In a more recent study, 90 PWIDs were recruited from three NEPs located in northeastern Ohio, Western Michigan, and southeastern Michigan in the United States, and participated in a structured interview that assessed barriers to conducting test shots and skin cleaning prior to injection (27). Although using clean needles was the most frequently reported safe injection behavior in this cohort, barriers to using clean needles were not assessed. 
Phillips (13) conducted a quantitative study examining barriers to using new/sterile needles with 48 heroin injectors in Denver, Colorado. With the exception of geographical location, our sample of rural Appalachian injectors shared several characteristics with the recruited urban Denver heroin injectors. Both samples were predominantly male (75\% in Denver; $57 \%$ in Appalachia) and included only a small proportion of African-American injectors (4\% in both studies). Both samples had a predominantly high school education (11.65 years of education, on average, in Denver and $78 \%$ with a high school education or less in our study), and in both samples, approximately 4 in 10 individuals reported being homeless. However, our sample had significantly more individuals identifying as Caucasian/White (96\% versus $57 \%$, respectively) and only one respondent identified as Hispanic. Although heroin was the most frequently cited drug of choice in our study, we did have a few respondents list an alternative drug of choice, which is different from the study in Denver that exclusively enrolled heroin injectors.

The overall number of barriers reported was slightly larger in the Denver sample compared to our study (average of 7.19 (SD, 3.62) barriers reported versus 6.02 (SD, 4.61), respectively). However, similar to our findings, only a small proportion of barriers was endorsed by at least $50 \%$ of respondents. More specifically, only 5 of 20 barriers were endorsed by more than $50 \%$ of respondents in Denver, and only 3 of 20 barriers were endorsed by more than $50 \%$ in our rural setting, although an additional 2 barriers related to difficulties purchasing clean syringes at a pharmacy (items 15, 16) were endorsed by almost half of our sample. 
Notably, and congruent with our findings, fear of arrest for possession of clean needles was the most frequently cited barrier in Denver ( $98 \%$ of injectors, 45 of 48 ). This finding is also congruent with other studies. A recent qualitative case study of rural Appalachian NEPs by Davis et al. (14) observed a legal conundrum created by paraphernalia laws that manifested in PWIDs being cited for clean needle possessions. In a study examining self-reported reasons for needle sharing and not carrying bleach among PWIDs in Baltimore Maryland, $69 \%$ of participants reported knowing a fellow injector who had "gotten in trouble" by law enforcement for carrying needles (23). Furthermore, such fear of arrest may preclude exchange attendance to obtain clean needles. An earlier study conducted with PWIDs attending NEPs in Rhode Island also observed fear of identification and/or police harassment as a significant barrier to exchange participation (28). Consequently, the success of NEPs is directly contingent on close cooperation between law enforcement and public health officials. In particular, Beletsky (29) noted a discrepancy between the "law on the books" (i.e., legality of syringe possession and exchange) and the "law on the streets" (i.e., discretionary behavior of law enforcement in the form of arrests and citations for needle possession)(29). Therefore, a better understanding of law enforcement attitudes underlying variable enforcement behaviors is indicated. Elucidation of these attitudes can form the basis of tailored interventions to promote law enforcement acceptance of NEPs at a local or regional level to improve success and acceptability (30).

There is a dearth of literature regarding underlying police attitudes toward NEPs that may influence policing behaviors, particularly PWID arrests and syringe confiscations, that have been shown to increase the odds of receptive syringe sharing 
(31). A qualitative study of 14 police officers in Roade Island revealed negative attitudes toward syringe access programs primarily in the form of a belief that such programs enable injection drug use (29). However, significant anxieties over transmission of disease (i.e., HIV, HCV) via needlestick injuries were also observed. A more recent qualitative case study observed similar law enforcement anxieties over needlestick injuries, and suggested that NEP acceptance increased when police were educated on the prevention of blood borne viruses benefit of needle exchange and viewed these programs as a blood-borne intervention versus a drug intervention (14). Indeed, a seminal intervention with police officers in Rhode Island aimed at increasing understanding, awareness, and acceptance of syringe access programs suggested that occupational safety training could form the basis of a successful law enforcement intervention that aligns law enforcement with the public health goals of NEPs (30).

Interestingly, the difficulties related to purchasing syringes at pharmacies observed in our study was not reported in the Denver sample. According to a colleague from the School of Pharmacy, it is not illegal for pharmacists to sell syringes without a prescription in West Virginia. However, these sales are left to the discretion of pharmacists who often have to weigh the public health goal of preventing infectious disease with concerns over endorsement of drug use, which may contribute to practice variability (Garofoli M, e-mail communication, 7 Nov, 2017). Such variability has been documented in the United States with syringe purchase refusal rates between $23 \%$ and $79 \%$ observed in states where nonprescription sale of syringes by pharmacies is legal (32-34). Concerns over endorsement of drug use and stigmatization of drug users were among the factors associated with these refusals (32). As a result of these findings, 
there has been a call to pass legislation that explicitly mandates pharmacy sales of nonprescription syringes as opposed to current discretionary practices $(32,35)$. Education of pharmacists to promote awareness of existing state laws that allow nonprescription sales of clean needles and the efficacy of clean needle use in preventing infectious diseases has been advanced $(32,35)$. Pharmacy policies that explicitly promote nonprescription syringe sales have been encouraged (36).

Finally, we did not find being in withdrawal to be a barrier to using clean needles in our rural sample as was observed in Denver. Whereas only one quarter of our respondents cited being in withdrawal as a barrier to using clean needles in our setting, $65 \%$ either agreed or strongly agreed that this was a barrier in Denver, Colorado. Potential explanations for this discrepancy are unclear and warrants further investigation.

Our findings are subject to some limitations. The results may be subject to reporting bias, specifically in the form of social desirability bias. However, we employed an anonymous survey design to minimize this source of bias. Additionally, given the hard-to-reach PWID population, we were not able to employ a random sampling scheme, which may have biased our results. Such bias could affect the generalizability of our findings both to other injectors attending the two NEP sites in our study and also to PWIDs attending other NEPs located within West Virginia (8 additional NEPs have opened in West Virginia (37) since the first two that were selected for this study). Furthermore, these results may not be applicable to PWIDs in other locales outside of the rural, Appalachian environment or even to other PWIDs in West Virginia. These limitations notwithstanding, we were able to achieve a sample double the size of the 
only other existing empirical study on potential barriers to using clean needles. Future studies should seek to confirm these results in other locales and specifically examine the impact of paraphernalia laws and policing behaviors on the efficacy of the needle exchange model.

\section{CONCLUSIONS}

Fear of arrest for possessing clean needles and difficulties with pharmacy purchase of clean needles were the most commonly cited barriers to using new needles among our population of injection drug users attending two needle exchange programs in rural West Virginia. Future studies should confirm these results in other rural settings and explore the design and implementation of individual and structural interventions with law enforcement officials and pharmacists that promote access to sterile syringes.

\section{REFERENCES}

1. CDC. Viral Hepatitis Surveillance - United States, 2015. [cited 26 Sept 2017]. Available from: https://www.cdc.gov/hepatitis/statistics/2015surveillance/commentary.htm.

2. Ward JW. The hidden epidemic of hepatitis $C$ virus infection in the United States: occult transmission and burden of disease. Top Antivir Med. 2013;21(1):15-9.

3. Schoener EP, Hopper JA, Pierre JD. Injection drug use in North America. Infectious Disease Clinics.16(3):535-51.

4. Suryaprasad AG, White JZ, Xu F, Eichler B-A, Hamilton J, Patel A, et al. Emerging Epidemic of Hepatitis C Virus Infections Among Young Nonurban Persons Who Inject Drugs in the United States, 2006-2012. Clinical Infectious Diseases. 2014;59(10):1411-9.

5. Zibbell JE, Iqbal K, Patel RC, Suryaprasad A, Sanders KJ, Moore-Moravian L, et al. Increases in hepatitis $C$ virus infection related to injection drug use among persons aged $</=30$ years - Kentucky, Tennessee, Virginia, and West Virginia, 2006-2012. MMWR Morb Mortal Wkly Rep. 2015;64(17):453-8. 6. Havens JR, Lofwall MR, Frost SDW, Oser CB, Leukefeld CG, Crosby RA. Individual and Network Factors Associated With Prevalent Hepatitis C Infection Among Rural Appalachian Injection Drug Users. American Journal of Public Health. 2013;103(1):e44-e52.

7. Moody L, Satterwhite E, Bickel WK. Substance Use in Rural Central Appalachia: Current Status and Treatment Considerations. Rural mental health. 2017;41(2):123-35.

8. Fraser H, Zibbell J, Hoerger T, Hariri S, Vellozzi C, Martin NK, et al. Scaling-up HCV prevention and treatment interventions in rural United States- model projections for tackling an increasing epidemic. Addiction. 2017.

9. Des Jarlais DC. Harm reduction in the USA: the research perspective and an archive to David Purchase. Harm Reduct J. 2017;14(1):51. 
10. Zibbell JE, Asher AK, Patel RC, Kupronis B, Iqbal K, Ward JW, et al. Increases in Acute Hepatitis C Virus Infection Related to a Growing Opioid Epidemic and Associated Injection Drug Use, United States, 2004 to 2014. Am J Public Health. 2018;108(2):175-81.

11. Pouget ER, Hagan H, Des Jarlais DC. Meta-analysis of hepatitis C seroconversion in relation to shared syringes and drug preparation equipment. Addiction. 2012;107(6):1057-65.

12. Fernandes RM, Cary M, Duarte G, Jesus G, Alarcao J, Torre C, et al. Effectiveness of needle and syringe Programmes in people who inject drugs - An overview of systematic reviews. BMC Public Health. 2017;17(1):309.

13. Phillips KT. Barriers to practicing risk reduction strategies among people who inject drugs. Addiction Research \& Theory. 2015;24(1):62-8.

14. Davis SM. Needle exchange programs to prevent hepatitis c virus infection in people who inject drugs in rural Appalachia [dissertation]. Morgantown, WV; West Virginia University; 2018.

15. City of Huntington. Cabell-Huntington Health Department launches syringe exchange 2015. [cited 2017 Nov 5]. Available from: http://www.cityofhuntington.com/news/view/cabell-huntingtonhealth-department-launches-syringe-exchange.

16. Milan Puskar Health RIGHT. LIGHT Program: Living In Good Health Together (LIGHT). [cited 5 Nov 2017]. Available from: https://mphealthright.org/light-program/.

17. Australian Bureau of Statistics. Pre-Testing in Survey Development: An Australian Bureau of Statistics Perspective; 2001. [cited 5 Nov 2017]. Available from:

http://www.nss.gov.au/nss/home.NSF/75427d7291fa0145ca2571340022a2ad/906dca4c30eac6aeca257 1ab0024717c/\$FILE/Pre-Testing\%20in\%20Survey\%20Development.pdf.

18. Fowler F. Evaluating survey questions and instruments. . Survey Research Methods. 5th ed. Thousand Oaks, CA: Sage Publications, Inc.; 2014. p. 99-109.

19. Fowler F. Designing questions to be good measures. . Survey Research Methods. 5th ed. Thousand Oaks, CA: Sage Publications, Inc.; 2014. p. 75-98.

20. Fowler F. Implementing a sample design. 5th ed. Thousand Oaks, CA: Sage Publications, Inc.; 2014. p. 42-60.

21. $\quad$ SAS Institute I. JMP Pro 13.0. Carey, NC: SAS Institute, Inc.; 2017.

22. Williams AB. Women at risk: an AIDS educational needs assessment. Image--the journal of nursing scholarship. 1991;23(4):208-13.

23. Latkin CA, Mandell W, Vlahov D, Knowlton AR, Oziemkowska M, Celentano DD. Self-Reported Reasons for Needle Sharing and Not Carrying Bleach among Injection Drug Users in Baltimore, Maryland. Journal of Drug Issues. 1995;25(4):865-70.

24. Gleghorn AA, Corby NH. Injection Drug Users' Reactions to Guidelines for Bleach Disinfection of Needles and Syringes: Implications for HIV Prevention. Journal of Drug Issues. 1996;26(4):865-81.

25. Hagan H, Pouget ER, Des Jarlais DC. A Systematic Review and Meta-Analysis of Interventions to Prevent Hepatitis C Virus Infection in People Who Inject Drugs. Journal of Infectious Diseases.

2011;204(1):74-83.

26. CDC. Hepatitis C \& Injection Drug Use; 2016. [cited 3 Dec 2017]. Available from:

https://www.cdc.gov/hepatitis/hcv/pdfs/factsheet-pwid.pdf.

27. Bonar EE, Rosenberg H. Injection Drug Users' Perceived Barriers to Using Self-Initiated Harm Reduction Strategies. Addict Res Theory. 2014;22(4):271-8.

28. Rich JD, Strong L, Towe CW, McKenzie M. Obstacles to needle exchange participation in Rhode Island. J Acquir Immune Defic Syndr. 1999;21(5):396-400.

29. Beletsky L, Macalino GE, Burris S. Attitudes of police officers towards syringe access, occupational needle-sticks, and drug use: A qualitative study of one city police department in the United States. International Journal of Drug Policy. 2005;16(4):267-74. 
30. Beletsky L, Agrawal A, Moreau B, Kumar P, Weiss-Laxer N, Heimer R. Police training to align law enforcement and HIV prevention: preliminary evidence from the field. Am J Public Health. 2011;101(11):2012-5.

31. Pollini RA, Brouwer KC, Lozada RM, Ramos R, Cruz MF, Magis-Rodriguez C, et al. Syringe possession arrests are associated with receptive syringe sharing in two Mexico-US border cities. Addiction. 2008;103(1):101-8.

32. Pollini RA, Rudolph AE, Case P. Nonprescription syringe sales: a missed opportunity for HIV prevention in California. Journal of the American Pharmacists Association : JAPhA. 2015;55(1):31-40.

33. Compton WM, Horton JC, Cottler LB, Booth R, Leukefeld CG, Singer M, et al. A multistate trial of pharmacy syringe purchase. Journal of urban health : bulletin of the New York Academy of Medicine. 2004;81(4):661-70.

34. Lutnick A, Cooper E, Dodson C, Bluthenthal R, Kral AH. Pharmacy Syringe Purchase Test of Nonprescription Syringe Sales in San Francisco and Los Angeles in 2010. Journal of urban health : bulletin of the New York Academy of Medicine. 2013;90(2):276-83.

35. Janulis P. Pharmacy nonprescription syringe distribution and HIV/AIDS: a review. Journal of the American Pharmacists Association : JAPhA. 2012;52(6):787-97.

36. Deibert RJ, Goldbaum G, Parker TR, Hagan H, Marks R, Hanrahan M, et al. Increased Access to Unrestricted Pharmacy Sales of Syringes in Seattle-King County, Washington: Structural and IndividualLevel Changes, 1996 Versus 2003. American Journal of Public Health. 2006;96(8):1347-53.

37. North American Syringe Exchange Network. Directory of Syringe Exchange Programs. [cited 2 Jan 2018]. Available from: https://nasen.org/directory/wv/s. 
Table 1. Participant Characteristics

\begin{tabular}{|c|c|c|c|c|c|c|}
\hline \multirow[b]{2}{*}{ Demographics } & \multicolumn{2}{|c|}{$\underset{(n=100)}{\text { All }}$} & \multirow[t]{2}{*}{$\begin{array}{c}\text { Health } \\
\text { Department } \\
(n=74)\end{array}$} & \multicolumn{2}{|c|}{$\begin{array}{l}\text { LIGHT } \\
\text { Program } \\
(n=26)\end{array}$} & \multirow[t]{2}{*}{$P^{*}$} \\
\hline & & & & & & \\
\hline \multirow[t]{2}{*}{ Age (avg.) } & \multicolumn{2}{|c|}{37.05} & 37.97 & \multicolumn{2}{|c|}{34.42} & 0.1307 \\
\hline & $\%$ & $\mathrm{n}$ & $\%$ & $\mathrm{n}$ & $\%$ & \\
\hline \multicolumn{7}{|l|}{ Gender } \\
\hline Female & 43 & 33 & 44.60 & 10 & 38.46 & \\
\hline Male & 57 & 41 & 55.40 & 16 & 61.54 & 0.5869 \\
\hline \multicolumn{7}{|l|}{ Education } \\
\hline$<$ High School & 21 & 19 & 25.68 & 2 & 7.69 & \\
\hline High School Graduate & 57 & 39 & 52.70 & 18 & 69.23 & \\
\hline$>$ High School & 22 & 16 & 21.62 & 6 & 23.08 & 0.1420 \\
\hline \multicolumn{7}{|l|}{ Race } \\
\hline White & 96 & 71 & 95.95 & 25 & 96.15 & \\
\hline Black/African American & 4 & 3 & 4.05 & 1 & 3.85 & 1.000 \\
\hline \multicolumn{7}{|l|}{ Hispanic } \\
\hline Yes & 1 & 1 & 1.35 & 0 & 0 & \\
\hline No & 98 & 72 & 97.30 & 26 & 100 & \\
\hline Missing & 1 & 1 & 1.35 & 0 & 0 & 1.000 \\
\hline \multicolumn{7}{|l|}{ Employment Status } \\
\hline Employed & 22 & 15 & 20.27 & 7 & 26.92 & \\
\hline Unemployed & 42 & 30 & 40.54 & 12 & 46.15 & \\
\hline Unable to Work & 24 & 20 & 27.03 & 4 & 15.39 & \\
\hline Other (e.g., Student) & 12 & 9 & 12.16 & 3 & 11.54 & 0.6539 \\
\hline \multicolumn{7}{|l|}{ Homeless } \\
\hline Yes & 40 & 33 & 44.60 & 7 & 26.92 & \\
\hline No & 60 & 41 & 55.40 & 19 & 73.08 & 0.1136 \\
\hline \multicolumn{7}{|l|}{ Hepatitis C Positive } \\
\hline Yes & 32 & 25 & 33.78 & 7 & 26.92 & \\
\hline No & 58 & 41 & 55.41 & 17 & 65.39 & \\
\hline Don't Know & 10 & 8 & 10.81 & 2 & 7.69 & 0.6702 \\
\hline \multicolumn{7}{|l|}{ Injection History } \\
\hline Daily Injections (median) & \multicolumn{2}{|c|}{5} & 5 & \multicolumn{2}{|c|}{5} & 0.6776 \\
\hline Weekly Injections (median) & \multicolumn{2}{|c|}{30} & 30 & \multicolumn{2}{|c|}{31.25} & 0.5678 \\
\hline Duration Injecting (yrs.) & \multicolumn{2}{|c|}{4} & 4 & \multicolumn{2}{|c|}{5.25} & 0.0612 \\
\hline \multicolumn{7}{|l|}{ Barriers to Clean Needle Use } \\
\hline \# of Barriers (median) & \multicolumn{2}{|c|}{5} & 5.5 & \multicolumn{2}{|c|}{5} & 0.7227 \\
\hline $\begin{array}{l}\text { Most Frequently Reported } \\
\text { Barrier }\end{array}$ & \multicolumn{2}{|c|}{$\begin{array}{l}\text { Fear of } \\
\text { Arrest }\end{array}$} & $\begin{array}{l}\text { Fear of } \\
\text { Arrest }\end{array}$ & & $\begin{array}{l}\text { r of } \\
\text { est }\end{array}$ & \\
\hline${ }^{\star}$ Test for the difference between the He & partm & it an & LIGHT Prog & & & \\
\hline
\end{tabular}


Table 2. Reported Barriers to Using New Needles by People Who Inject Drugs $(N=100)$

\begin{tabular}{|c|c|c|}
\hline & Agree & Disagree/Neutral \\
\hline & $\%$ & $\%$ \\
\hline 1. It takes too long to get a new needle every time I inject. & 15 & 85 \\
\hline 2. It is inconvenient to get a new needle every time I inject. & 24.74 & 75.26 \\
\hline $\begin{array}{l}\text { 3. I often don't want to take the time to get a new needle because my cravings or } \\
\text { urges to use drugs are too strong. }\end{array}$ & 18.37 & 81.63 \\
\hline 4. I often do not take the time to get a new needle if I am drug sick or in withdrawal. & 24.45 & 75.55 \\
\hline $\begin{array}{l}\text { 5. I don't take the time to get a new needle before injecting because I can only think } \\
\text { about getting high. }\end{array}$ & 23.23 & 76.77 \\
\hline $\begin{array}{l}\text { 6. I don't take the time to get a new needle before injecting if I'm already high or } \\
\text { drunk. }\end{array}$ & 19.39 & 80.61 \\
\hline 7. The places where I inject usually do not have access to new needles. & 29.29 & 70.71 \\
\hline $\begin{array}{l}\text { 8. If I am in a shooting gallery (a place where people inject drugs, "dope den", "joy } \\
\text { popping", etc.), I often do not use a new needle. }\end{array}$ & 21.74 & 78.26 \\
\hline 9. I often do not carry new needles with me when I'm out. & 30.93 & 69.07 \\
\hline 10. There isn't a needle exchange close by for me to get needles. & 16.67 & 83.33 \\
\hline 11. Pharmacies sometimes give me hassle when I try to buy needles. & 63.33 & 36.37 \\
\hline $\begin{array}{l}\text { 12. After I inject, I don't prepare in advance by getting new needles ready for my next } \\
\text { injection. }\end{array}$ & 39.80 & 60.20 \\
\hline 13. It's too expensive to buy new needles from the pharmacy for every time I inject. & 55.91 & 44.09 \\
\hline $\begin{array}{l}\text { 14. Feeling sad or depressed would get in the way of my using a new needle every } \\
\text { time I inject. }\end{array}$ & 20.21 & 79.79 \\
\hline 15. It is embarrassing to buy needles at the pharmacy. & 47.92 & 52.08 \\
\hline $\begin{array}{l}\text { 16. I worry that someone (friends, family, etc.) may see me buying needles at the } \\
\text { pharmacy. }\end{array}$ & 49.47 & 50.53 \\
\hline 17. My peers/friends would look at me funny if I used a new needle every time I inject. & 17.53 & 82.47 \\
\hline 18. Having to worry about using a new needle interrupts the ritual of using. & 16.84 & 83.16 \\
\hline 19. I am unlikely to use a new needle if a friend lets me borrow his or her used needle. & 19.59 & 80.41 \\
\hline 20. I could get in trouble from the police if I carry needles around with me & 72.00 & 28.00 \\
\hline
\end{tabular}




\section{SUMMARY OF FINDINGS}

\subsection{Study 1}

\subsubsection{Findings}

An updated systematic review with meta-analysis (specific aim \#1) yielded mixed results with regard to the efficacy of needle exchange programs for the prevention of hepatitis $\mathrm{C}$ in people who inject drugs. Results from studies yielding odds ratio (OR) estimates indicated no effect on the odds of HCV seroconversion from NEP participation (OR, 0.51, 95\% Cl, 0.05-5.15); whereas, pooled results from studies yielding hazard ratio $(\mathrm{HR})$ estimates indicated a harmful effect $(\mathrm{HR}, 2.05,95 \% \mathrm{Cl}, 1.39-$ $3.03) .53$

\subsubsection{Discussion and Implications for Future Research}

The central finding of mixed evidence is likely due to substantial heterogeneity in study populations, interventions, comparisons, and outcomes. In particular, there is some evidence that selecting HCV seroconversion as the primary outcome of interest may not be the outcome that best demonstrates the potentially protective effect of NEPs. Rather, these programs have demonstrated substantially higher protective benefit in the form of a reduction in injection risk behavior (e.g., syringe sharing), ${ }^{17}$ which is the number one risk factor for HCV seroconversion. ${ }^{14}$ Future systematic reviews and meta-analyses may, therefore, wish to focus on syringe sharing as the primary outcome of interest. However, the aforementioned heterogeneity, especially in selected populations, specific intervention characteristics, and outcomes (and associated statistical analyses) may still preclude a pooling of meaningful results to 
obtain estimates of overall effect. Therefore, there is a need for well-designed, standardized studies to clarify the overall efficacy of NEP on the prevention of HCV.

With regard to the intervention itself, recent research suggests that the addition of opiate replacement therapy (ORT) that administers methadone or buprenorphine to needle exchange programs may more effectively prevent HCV seroconversion in PWIDs. One meta-analysis demonstrated that such combined strategies reduced the risk of HCV seroconversion by $75 \%$ (pooled RR $0.25,95 \% \mathrm{Cl}, 0.07-0.83$ ) compared to effects from single method interventions that had relative risks (RRs) ranging from 0.6 to 1.6. ${ }^{13}$ Similarly, a study pooling NEP plus ORT intervention results in the United Kingdom observed a $79 \%$ reduction in the odds of new HCV infection (aOR $0.21,95 \%$ $\mathrm{Cl}, 0.08-0.52),{ }^{54}$ and another study from Scotland observed a $71 \%$ reduction (aOR 0.29 , $95 \% \mathrm{Cl}, 0.11-0.74)$ in new $\mathrm{HCV}$ cases. ${ }^{55} \mathrm{~A}$ very recent systematic review with metaanalysis conducted by the Cochrane Collaboration found that combined ORT/NEP therapy was associated with a $76 \%$ reduction in $\mathrm{HCV}$ acquisition $(\mathrm{RR}=0.24,95 \% \mathrm{Cl}$, $0.07-0.89$ ) versus NEP alone, which was associated with increased HCV risk in North American studies $(\mathrm{RR}=1.58, \mathrm{P}=<0.001) .{ }^{56}$ Interestingly and in contrast, high needle and syringe program coverage demonstrated a protective effect in Europe ( $R R=0.44$, $95 \% \mathrm{Cl} 0.24-0.80)^{56}$ However, identical to the findings of study \#1, heterogeneity remained a significant problem in this meta-analysis, and the overall evidence was judged to be of low quality. Furthermore, because all of the combined intervention studies included in these meta-analyses were conducted in Europe where methadone is frequently used as an ORT, there is no current information on whether or not differential 
effects may exist for other opiate replacement therapies (i.e., buprenorphine) used in combination with NEP.

It is plausible that observed reductions in the overall odds of HCV seroconversion from the combined approach may be due to demonstrated reductions in overall drug use and risky injection behavior, the latter of which is the chief risk factor for HCV seroconversion in PWIDs. ${ }^{14}$ For example, Turner et al. ${ }^{54}$ observed an average reduction of 20.8 injections per month and a $48 \%$ reduction $(\mathrm{aOR} 0.52,95 \% \mathrm{Cl}, 0.32-$ 0.83) in the risk of needle sharing in PWIDs participating in full harm reduction (i.e., high NSP coverage and receiving ORT) in the United Kingdom. Additionally, during a time of scaled-up coverage of combined harm reduction interventions in Scotland, the proportion of PWIDs injecting daily declined $14 \%(p<0.001) .{ }^{55}$ However, more research is needed to clarify the overall impact of this combined strategy on prevention of HCV in PWIDs located in North America, where combined strategies are not widely available. Indeed, implementing combined NEP and ORT therapy may be challenged by the belief that opiate replacement therapy is intended to take an abstinence only approach (i.e., individuals on ORT should not be actively injecting drugs using needles obtained from a syringe access program). For example, a pilot NEP implemented in Washington, DC shared office space with a methadone program, and some staff of the ORT program were strongly opposed to needle distribution in a treatment setting. ${ }^{57}$ Therefore, any successful implementation of a combined strategy may require a substantial treatment paradigm shift. To this end, future studies should seek to measure the attitudes and prevailing beliefs of ORT staff members located in the United States regarding a combined approach to combating the HCV epidemic. Of note, and a 
potential area for future research, is the fact that recent mathematical modeling suggests that a reduction in HCV prevalence will be greatest when direct-acting antiviral therapies such as sofosbuvir that can effectively cure HCV are combined with a scale up in coverage of both ORT and NEP interventions. ${ }^{58}$

\subsubsection{Strengths and Weaknesses}

This study was the first update to the only existing previous study ${ }^{13}$ specifically examining the efficacy of NEP for the prevention of HCV, and included studies with designs that promoted reaching causal conclusions (i.e., cohort or case-control designs). However, the small number of studies that met our criteria precluded the assessment of small study effects (i.e., publication bias) and potential moderator effects. As with any meta-analysis, the biases inherent in individual studies (e.g., information bias, ${ }^{59}$ volunteer bias ${ }^{60,61}$ ) will also be present in the pooled results, and aggregate results may not be applicable to individuals (i.e., the ecological fallacy ${ }^{62}$ ).

This meta-analysis also excluded other sources of sterile needles such as supervised injection facilities (SIF) and pharmacies, which may increase the overall availability of clean needles available to PWIDs. ${ }^{63}$ However, as of 2017, SIFs were just opening in a few urban areas of the United States and none were present in the rural, Appalachian area that was the focus of this dissertation. Similarly, there were no pharmacy-based NEPs operating in West Virginia at the time of this dissertation, and very limited evidence exists ( $N=2$ studies) ${ }^{64}$ regarding the impact of pharmacy-based NEP on HCV prevention. Therefore, future studies should explore the addition of other sources of clean needles on the prevention of HCV among PWIDs in rural Appalachia. Finally, and similar to previous research, ${ }^{13}$ this systematic review did not locate any 
individual studies describing and examining the efficacy of NEP in rural areas. This absence of information formed the rationale for study 2 .

\subsection{Study 2}

\subsubsection{Findings}

A qualitative case study of two of the longest operating NEPs in the rural, Appalachian State of West Virginia (specific aim \#2) revealed common barriers and facilitators to program implementation, ongoing operations, and future expansion. Although broad community support was a noted strength by both programs, ongoing operations were threatened by unexpected program demand and funding pressures created by the federal government's ban on the use of funds to purchase needles. Structural barriers in the form of paraphernalia laws created a legal conundrum for attendees who reported criminal sanctions for the possession of needles, whether clean or used, which has been associated with increased syringe sharing.

\subsubsection{Discussion and Implications for Future Research}

The barriers and facilitators experienced by our small, rural Appalachian NEPs are similar to those reported by the first NEPs to open in the United States in urban areas such as Tacoma, Washington ${ }^{37,65}$; San Francisco, CA ${ }^{66}$; New York City 67 ; and, Washington, DC. ${ }^{57}$ However, there is some evidence that NEPs located in rural areas may experience more funding pressures than other geographical regions. A recent survey ${ }^{68}$ revealed that rural NEPs more frequently reported funding difficulties in comparison to their urban and suburban counterparts. The specific factors related to this disparity are not well understood. It is possible that these funding pressures are 
merely reflective of the fact that rural areas of the United States are poorer than more urban areas. ${ }^{69}$ In comparison to the rest of the United States, the Appalachian region is economically disadvantaged with lower life expectancies and higher mortality, ${ }^{70}$ and declining rural manufacturing and coal industry jobs have created areas that have been described as "chronically poor". ${ }^{71}$ Other factors such as the overwhelming volume of attendees created by the opioid and heroin epidemics in Central Appalachia may also play a role in funding shortfalls. Future studies examining factors driving this observed geographical funding disparity between urban, suburban and rural NEPs are indicated.

The community support noted as a significant facilitator by both programs was somewhat unexpected and in contrast to historical controversy over needle exchange programs in the United States. ${ }^{72}$ Such controversy is rooted in the fact that drug use has historically been treated as a moral failing in need of a criminal solution in the United States. ${ }^{72}$ Increasing acceptance of the use of illicit substances such as marijuana ${ }^{73}$ may, in part, explain a shift in community attitudes towards needle exchange. Even more conservative state and local governments have shown increasing support of NEPs in the face of infectious disease outbreaks of HIV and hepatitis C. ${ }^{74}$ Community support may also have been related to the spike in overdose deaths that precipitated the NEP implementation in one Appalachian community in this study. Given the strong facilitative role of community support observed in this case study, future research should directly examine factors related to support of the NEP model in rural communities. Increasing public support may translate into a change in federal policy to allow funds to be used for the purchase of sterile needles in addition to program operations. 
The conundrum reported by several participants, including PWIDs and law enforcement members, over the action that should be taken upon discovery of needles, whether clean or used, is of particular concern given the relationship between criminal sanctions and the spread of infectious disease. A study by Pollini et al. ${ }^{21}$ observed that arrests for needle possession were significantly associated with receptive syringe sharing, which is the number one risk factor for HCV seroconversion among PWIDs. ${ }^{14}$ Pipitpan et al. ${ }^{20}$ also observed an association between syringe confiscation and lower safe injection self- efficacy $(p=0.04)$. Whereas the action to be taken in the presence of clean needles is somewhat less clear, law enforcement members were unequivocal in their response to finding needles with drug residue (i.e., citation and/or arrest) in this qualitative case study. This observation is of concern given that it threatens the entire exchange model. If attendees fear potential arrest for possession of used needles, they may be less likely to bring used needles back to exchange for clean ones. However, attendee fear of arrest and other potential barriers to using clean needles among PWIDs living in rural Appalachia had not previously been reported, and formed the primary basis for the third and final study of this dissertation.

\subsubsection{Strengths and Weaknesses}

This study is the first qualitative case study of rural needle exchange programs located in central Appalachia. The implementation of qualitative research techniques yielded an in-depth understanding of barriers and facilitators to program implementation, ongoing operations, and future expansion from the viewpoints of program staff, law enforcement, and program attendees. Findings from this study may inform the implementation and operation of exchanges in other central Appalachian 
areas battling the current heroin epidemic in rural America. However, some study limitations should be noted. It is possible that responses may have been impacted by social desirability bias given the sensitive study topic. To promote unbiased responses, no names or other demographic information were collected. Given the convenience sample of respondents, it is also possible that the results observed in these two programs may not be transferable to other attendees within each program or other programs throughout the state. Therefore, future studies should examine the congruency of our findings with other programs in West Virginia and other central Appalachian locales.

\subsection{Study 3}

\subsubsection{Findings}

Findings from a cross-sectional survey of PWIDs attending two needle exchanges in West Virginia (specific aim \#3) suggest that there are significant structural barriers to the efficacy of NEPs. In specific, paraphernalia laws and concomitant policing behaviors (i.e., needle confiscation and/or arrest) may contribute to the inability of PWIDs to both bring used needles back for exchange and have clean needles available for use during every injection. Attendees also reported difficulties with purchasing needles at pharmacies without a prescription, which may be related to an absence of explicit state laws permitting such sales.

\subsubsection{Discussion and Implications for Future Research}

The central finding of fear of law enforcement sanctions (e.g., arrest, syringe confiscation, etc.) as a primary barrier to using a clean needle for every injection may 
threaten the efficacy of the needle exchange model in preventing infectious diseases (e.g., HCV) and is congruent with interview data gathered during the second aim of this dissertation. This central barrier may be driven by 1) confusion over existing paraphernalia laws; and, 2) law enforcement attitudes and experiences.

Paraphernalia laws that differ by state and locations within each state in the United States may create confusion among both PWIDs and law enforcement regarding the legality of syringe possession, whether clean or used. For example, in the United States, 28 states have either limited or removed previous laws prohibiting needle distribution, 17 have explicitly authorized NEPs, and 14 states have removed syringes from the definition of drug paraphernalia. ${ }^{75}$ Within this tangled web of legislation, over 200 NEPs are currently known to operate. ${ }^{75}$ Therefore, it is not surprising that law enforcement unawareness of laws that allow purchase and possession of needles in some locations have been documented. ${ }^{76,77}$ Additionally, existing studies examining police attitudes toward needle exchange programs highlight the difficulty reported by officers that are sworn to uphold the law (confusion and lack of awareness notwithstanding), on the one hand, with a program that some view as explicitly encouraging illegal behavior, on the other hand. ${ }^{76-79}$

There is some initial evidence ${ }^{76}$ that educational interventions combining occupational safety training with an understanding of the public health benefits of needle exchange may promote a better understanding of paraphernalia laws that allow possession of clean needles and more positive attitudes toward harm reduction programs among law enforcement officers. A preliminary, 30-minute in-service training with Rhode Island police officers combined public health content (i.e., the harm 
reduction model) with occupational safety information (i.e., needlestick risks), and observed positive posttest shifts in legal and occupational safety knowledge. ${ }^{76}$ After training, officers correctly understood that it was legal for PWIDs to possess clean syringes in their setting $(\mathrm{OR}=9.16,95 \% \mathrm{Cl}, 4.80-17.51) .{ }^{76}$ Participants were also significantly more likely to understand the low risk of HIV transmission in the event of a needlestick injury $(\mathrm{OR}=3.22,95 \% \mathrm{Cl}, 1.78-523)$. However, attitudes about harm reduction programs, were only slightly altered and observed increases were not statistically significant. Post-training, a $2 \%$ increase in the odds of believing that harm reduction programs do not promote drug use was observed $(\mathrm{OR}=1.02,95 \% \mathrm{Cl}, 0.59$ 1.76). ${ }^{76}$ Similarly, post intervention, a $17 \%$ increase in the odds of believing that harm reduction programs reduce the risk of needlestick injuries was observed $(\mathrm{OR}=1.17$, $95 \% \mathrm{Cl}, 0.68-2.03) .{ }^{76}$ The authors suggest that long-standing, entrenched cultural beliefs may necessitate the need for law enforcement champions to facilitate changing underlying attitudes. ${ }^{76}$ This suggestion is congruent with study 2 of this dissertation, which observed the significant facilitative role that law enforcement champions played in the implementation and ongoing operations of NEPs in two rural, Appalachian communities. Notably, these champions promoted the intervention as a blood-borne intervention versus a drug intervention and emphasized the importance linkage to drug cessation services. Therefore, it may be necessary to increase awareness among law enforcement officers that harm reduction programs provide linkage to care with the goal of ultimately serving as a gateway to recovery. However, further research is needed on the long-term effects of such educational interventions on the actual behaviors of law enforcement toward PWIDs found with either used or clean needles. 
A more recent study suggests that policing behaviors may be associated with actual experiences. Cepeda et al. ${ }^{78}$ surveyed over 1300 officers in Tijuana, Mexico and observed $38 \%$ higher odds of syringe confiscation among officers reporting needlestick injuries (OR 1.38, 95\% Cl, 1.02-1.87). Based on these results, the authors suggest that negative attitudes toward PWIDs stemming from previous needlestick injuries may lead to more syringe confiscations. ${ }^{78}$ Therefore, interventions that seek to reduce the odds of experiencing a needlestick injury, which is at the core of the occupational safety component of the previous intervention with Rhode Island officers, may reduce syringe confiscation behaviors, and is an area in need of further research. However, findings from this study also suggested that policing behaviors may be positively associated with underlying attitudes. Although not statistically significant, the odds of arresting a PWID were $22 \%$ less among officers who held a positive view of laws that treat addiction as a public health issue $(\mathrm{aOR}=0.78,95 \% \mathrm{Cl}, 0.59-1.03) .{ }^{78}$ Therefore, the public health component of the intervention conducted with Rhode Island law enforcement may also translate to policing behaviors that promote non-confiscation of needles, and is another area in need of further inquiry. Ultimately, the most successful interventions may balance increasing positive attitudes and decreasing negative occupational experiences. For example, police concerns over acquiring infectious diseases, especially HCV, via a needlestick injury have been shown to increase stress, lead to an exaggeration of actual seroconversion risk, and result in a hostile attitude toward PWIDs that may promote policing behaviors that decrease the effectiveness of harm reduction programs. ${ }^{80}$ However, the success of any intervention is moot in the absence of laws that decriminalize possession of both clean and used needles. One strategy 
implemented by a NEP in Study 2 was the use of biohazard containers for needle transportation. It is unclear whether this strategy could minimize the confiscation and/or legal sanctioning of PWIDs found with needles, and is an area ripe for further inquiry. As it currently stands, fear of arrest may blunt the effectiveness of needle exchange in our rural Appalachian program. The presence of these structural influences on the performance of the exchange model could possibly explain, in part, the discrepancy between European NEPs that have been shown to be protective against the acquisition of hepatitis C versus North American NEPs that have been shown to increase the risk of HCV as discussed above.

The trouble experienced by PWIDs with obtaining nonprescription syringes from pharmacies, although not directly impacting needle exchange programs, may have implications for the future role of pharmacies in serving as an additional source of sterile needles. Lack of explicit direction from both state policies and professional organizations creates a professional conundrum whereby pharmacists must weigh the public health benefit of nonprescription syringe sales with concerns over explicit encouragement of illegal activity. ${ }^{81,82}$ In some cases pharmacists may be unaware of the legality of nonprescription sales. ${ }^{83,84}$ In others, pharmacists have expressed concern over the impact that PWIDs purchasing syringes in stores may have on staff safety and other customers. ${ }^{83}$ Such concerns have led some pharmacists to support NEPs in their neighborhood while continuing to oppose nonprescription pharmacy sales of syringes. ${ }^{82,85}$ Therefore, interventions only targeting pharmacists' knowledge of the legality of nonprescription syringe sales may not necessarily result in increasing sales. ${ }^{83}$ There have also been calls for professional pharmaceutical organizations to explicitly 
endorse the nonprescription sales of sterile syringes, ${ }^{84}$ and explicit state laws mandating such sales may promote access to clean syringes. ${ }^{83}$

\subsubsection{Strengths and Weaknesses}

This study used a psychometrically validated survey and obtained a sample size double the only other existing published study ${ }^{19}$ surveying PWIDs attending a needle exchange program on potential barriers to using a clean needle for every injection. However, social desirability bias is a noted limitation of our findings given the sensitivity of the survey questions. To minimize this bias, we made the survey entirely anonymous. Given the hard-to-reach PWID population, we were also only able to obtain a convenience sample of attendees. This sampling scheme may limit the generalizability of our findings to both attendees at the two sites surveyed in West Virginia and to attendees in other rural locations. Future studies should seek to confirm these results in other locales and specifically examine the impact of paraphernalia laws and policing behaviors on the efficacy of the needle exchange model.

\section{CONCLUSION}

People who inject drugs living in rural communities, particularly those located in the Central Appalachian region of the United States, continued to be a primary driver of the overall Hepatitis C Virus epidemic in the United States. However, these communities lag far behind their suburban and urban counterparts with regard to the availability of needle exchange programs. ${ }^{68}$ The results of this dissertation suggest that NEPs opened in rural Central Appalachia in response to an opioid and heroin epidemic have enjoyed robust community support and are viewed as an important infectious 
diseases prevention mechanism by people who inject drugs in the rural Appalachian communities examined. However, the overall impact of NEPs on preventing HCV in rural injection drug users is unclear and, similar to urban areas of the United States, may be blunted by legal structural influences (i.e. paraphernalia laws) that impact policing behaviors. Future studies should explore the factors associated with these structural barriers that may prevent the ability of rural PWIDs to use a clean needle during every injection, which, in turn, may minimize the overall efficacy of these programs on HCV prevention. Further research is also needed on the feasibility of implementing the addition of opiate replacement therapy with needle exchange programs given the strong protective effect on HCV acquisition among PWIDs observed in several European studies consistently over time. 


\section{REFERENCES SECTIONS 1, 2, 4 \& 5}

1. CDC. Viral Hepatitis. 2017; https://www.cdc.gov/hepatitis/hcv/. Accessed 12/16, 2017.

2. Ward JW. The hidden epidemic of hepatitis $C$ virus infection in the United States: occult transmission and burden of disease. Top Antivir Med. 2013;21(1):15-19.

3. Razavi $\mathrm{H}$, ElKhoury AC, Elbasha $\mathrm{E}$, et al. Chronic hepatitis $\mathrm{C}$ virus (HCV) disease burden and cost in the United States. Hepatology. 2013;57(6):2164-2170.

4. Ly KN, Xing J, Klevens RM, Jiles RB, Ward JW, Holmberg SD. The Increasing Burden of Mortality From Viral Hepatitis in the United States Between 1999 and 2007. Annals of Internal Medicine. 2012;156(4):271.

5. Rein DB, Wittenborn JS, Weinbaum CM, Sabin M, Smith BD, Lesesne SB. Forecasting the morbidity and mortality associated with prevalent cases of pre-cirrhotic chronic hepatitis $\mathrm{C}$ in the United Stated. Digestive and Liver Disease. 2011;43(1):66-72.

6. Galbraith JW, Donnelly JP, Franco RA, Overton ET, Rodgers JB, Wang HE. National Estimates of Healthcare Utilization by Individuals With Hepatitis C Virus Infection in the United States. Clinical Infectious Diseases. 2014;59(6):755-764.

7. CDC. Viral Hepatitis Surveillance - United States, 2015. 2017. https://www.cdc.gov/hepatitis/statistics/2015surveillance/commentary.htm. Accessed 9/26/2017.

8. Zibbell JE, Iqbal $\mathrm{K}$, Patel $\mathrm{RC}$, et al. Increases in hepatitis $\mathrm{C}$ virus infection related to injection drug use among persons aged $</=30$ years - Kentucky, Tennessee, Virginia, and West Virginia, 20062012. MMWR Morb Mortal Wkly Rep. 2015;64(17):453-458.

9. Page K, Morris MD, Hahn JA, Maher L, Prins M. Injection Drug Use and Hepatitis C Virus Infection in Young Adult Injectors: Using Evidence to Inform Comprehensive Prevention. Clinical Infectious Diseases. 2013;57(suppl_2):S32-S38.

10. Jordan $\mathrm{AE}$, Jarlais DD, Hagan $\mathrm{H}$. Prescription opioid misuse and its relation to injection drug use and hepatitis $C$ virus infection: protocol for a systematic review and meta-analysis. Systematic Reviews. 2014;3(1).

11. Suryaprasad AG, White JZ, Xu F, et al. Emerging Epidemic of Hepatitis C Virus Infections Among Young Nonurban Persons Who Inject Drugs in the United States, 2006-2012. Clinical Infectious Diseases. 2014;59(10):1411-1419.

12. Havens JR, Lofwall MR, Frost SDW, Oser CB, Leukefeld CG, Crosby RA. Individual and Network Factors Associated With Prevalent Hepatitis C Infection Among Rural Appalachian Injection Drug Users. American Journal of Public Health. 2013;103(1):e44-e52.

13. Hagan H, Pouget ER, Des Jarlais DC. A Systematic Review and Meta-Analysis of Interventions to Prevent Hepatitis C Virus Infection in People Who Inject Drugs. Journal of Infectious Diseases. 2011;204(1):74-83.

14. Pouget ER, Hagan H, Des Jarlais DC. Meta-analysis of hepatitis C seroconversion in relation to shared syringes and drug preparation equipment. Addiction. 2012;107(6):1057-1065.

15. Beheshti A, Lucas L, Dunz T, et al. An Evaluation of Naloxone Use for Opioid Overdoses in West Virginia: A Literature Review. Am Med J. 2015;6(1):9-13.

16. Hedrich D, Hartnoll R. Harm Reduction Interventions. Textbook of Addiction Treatment: International Perspectives: Springer Milan; 2014:1291-1313.

17. MacArthur GJ, van Velzen E, Palmateer N, et al. Interventions to prevent HIV and Hepatitis C in people who inject drugs: A review of reviews to assess evidence of effectiveness. International Journal of Drug Policy. 2014;25(1):34-52. 
18. Abdul-Quader AS, Feelemyer J, Modi S, et al. Effectiveness of Structural-Level Needle/Syringe Programs to Reduce HCV and HIV Infection Among People Who Inject Drugs: A Systematic Review. AIDS and Behavior. 2013;17(9):2878-2892.

19. Phillips KT. Barriers to practicing risk reduction strategies among people who inject drugs. Addiction Research \& Theory. 2015;24(1):62-68.

20. Pitpitan EV, Patterson TL, Abramovitz D, et al. Policing behaviors, safe injection self-efficacy, and intervening on injection risks: Moderated mediation results from a randomized trial. Health psychology : official journal of the Division of Health Psychology, American Psychological Association. 2016;35(1):87-91.

21. Pollini RA, Brouwer KC, Lozada RM, et al. Syringe possession arrests are associated with receptive syringe sharing in two Mexico-US border cities. Addiction. 2008;103(1):101-108.

22. Moher D, Liberati A, Tetzlaff J, Altman DG. Preferred Reporting Items for Systematic Reviews and Meta-Analyses: The PRISMA Statement. PLoS Medicine. 2009;6(7):e1000097.

23. van Driel ML, De Sutter A, De Maeseneer J, Christiaens T. Searching for unpublished trials in Cochrane reviews may not be worth the effort. Journal of Clinical Epidemiology. 2009;62(8):838844.e833.

24. Egger M, Juni P, Bartlett C, Holenstein F, Sterne J. How important are comprehensive literature searches and the assessment of trial quality in systematic reviews? Empirical study. Health technology assessment (Winchester, England). 2003;7(1):1-76.

25. Stern RK, Hagan H, Lelutiu-Weinberger C, et al. The HCV Synthesis Project: Scope, methodology, and preliminary results. BMC Medical Research Methodology. 2008;8(1).

26. Lee E, Dobbins M, DeCorby K, McRae L, Tirilis D, Husson H. An optimal search filter for retrieving systematic reviews and meta-analyses. BMC Medical Research Methodology. 2012;12(1).

27. Kali A, Srirangaraj S. EndNote as document manager for summative assessment. Journal of Postgraduate Medicine. 2016;62(2):124.

28. Margulis AV, Pladevall M, Riera-Guardia N, et al. Quality assessment of observational studies in a drug-safety systematic review, comparison of two tools: the Newcastle-Ottawa Scale and the RTI item bank. Clinical Epidemiology. 2014;6:359-368.

29. Ahn S, Becker BJ. Incorporating Quality Scores in Meta-Analysis. Journal of Educational and Behavioral Statistics. 2011;36(5):555-585.

30. Higgins JPT, Green S. Cochrane Handbook for Systematic Reviews of Interventions Version 5.1.0 [updated March 2011]. The Cochrane Collaboration; 2011: http://handbook.cochrane.org. Accessed 12/16/2017.

31. DerSimonian R, Laird N. Meta-analysis in clinical trials. Controlled Clinical Trials. 1986;7(3):177188.

32. Higgins JPT. Measuring inconsistency in meta-analyses. BMJ. 2003;327(7414):557-560.

33. Comprehensive meta-analysis. (3.0). [computer program]. Englewood, NJ: Biostat, Inc.; 2016.

34. Stake R. The Art of Case Study Research. Tousand Oaks, CA: Sage; 1995.

35. Creswell JW. Qualitative Inquiry and Research Design: Choosing Among Five Approaches. 3rd ed. Los Angeles, CA: Sage; 2013.

36. Miles MB. Qualitative Data Analysis: A Sourcebook of New Methods. 2nd ed. Thousand Oaks, CA: Sage; 1994.

37. Sherman SG, Purchase D. Point Defiance: a case study of the United States' first public needle exchange in Tacoma, Washington. International Journal of Drug Policy. 2001;12(1):45-57.

38. Denzin NK, Lincoln, Y.S. Introduction: The Discipline and Practice of Qualitative Research. 4th ed. Thousand Oaks, CA: Sage; 2011.

39. TASCAM. TASCAM DR-05. 2016; http://tascam-ca.com/product/dr-05/. Accessed 4/18/2016.

40. Yin RK. Case Study Research: Design and Methods. 5th. ed. Thousand Oaks, CA: Sage; 2016. 
41. NVIVO. NVIVO Software. 2016; http://www.qsrinternational.com/product Accessed 4/18/16.

42. Brinkmann S, Kvale S. Interviews: Learning the Craft of Qualitative Research Interviewing. 3rd ed. Thousand Oaks, CA: Sage; 2015.

43. Harry B. Mapping the process: an exemplar of process and challenge in grounded theory analysis. Educational Researcher. 2005;34(2):3-13.

44. Sandelowski M, Barroso, J. Handbook for Synthesizing Qualitative Research. New York, NY: Springer; 2007.

45. Saldana J. The Coding Manual for Qualitative Researchers. 3rd ed. Thousand Oaks, CA: Sage; 2016.

46. Atchan M, Davis D, Foureur M. A methodological review of qualitative case study methodology in midwifery research. J Adv Nurs. 2016;72(10):2259-2271.

47. Tong A, Sainsbury P, Craig J. Consolidated criteria for reporting qualitative research (COREQ): a 32-item checklist for interviews and focus groups. Int J Qual Health Care. 2007;19(6):349-357.

48. Fowler F. Designing questions to be good measures. . Survey Research Methods. 5th ed. Thousand Oaks, CA: Sage Publications, Inc.; 2014:75-98.

49. Statistics ABo. Pre-Testing in Survey Development: An Australian Bureau of Statistics Perspective. 2001.

http://www.nss.gov.au/nss/home.NSF/75427d7291fa0145ca2571340022a2ad/906dca4c30eac6 aeca2571ab0024717c/\$FILE/Pre-Testing\%20in\%20Survey\%20Development.pdf. Accessed $11 / 5 / 2017$.

50. Fowler F. Evaluating survey questions and instruments. . Survey Research Methods. 5th ed. Thousand Oaks, CA: Sage Publications, Inc.; 2014:99-109.

51. Fowler F. Implementing a sample design. 5th ed. Thousand Oaks, CA: Sage Publications, Inc.; 2014:42-60.

52. SAS Institute I. JMP Pro 13.0. Carey, NC: SAS Institute, Inc.; 2017.

53. Davis SM, Daily S, Kristjansson AL, et al. Needle exchange programs for the prevention of hepatitis $C$ virus infection in people who inject drugs: a systematic review with meta-analysis. Harm Reduct J. 2017;14(1):25.

54. Turner KME, Hutchinson $S$, Vickerman $P$, et al. The impact of needle and syringe provision and opiate substitution therapy on the incidence of hepatitis $C$ virus in injecting drug users: pooling of UK evidence. Addiction. 2011;106(11):1978-1988.

55. Palmateer NE, Taylor A, Goldberg DJ, et al. Rapid decline in HCV incidence among people who inject drugs associated with national scale-up in coverage of a combination of harm reduction interventions. PLoS One. 2014;9(8):e104515.

56. Platt L, Minozzi S, Reed J, et al. Needle syringe programmes and opioid substitution therapy for preventing HCV transmission among people who inject drugs: findings from a Cochrane Review and meta-analysis. Addiction. 2017.

57. Vlahov D, Ryan C, Solomon L, Cohn S, Holt MR, Akhter MN. A pilot syringe exchange program in Washington, DC. Am J Public Health. 1994;84(2):303-304.

58. Grebely J, Dore GJ, Morin S, Rockstroh JK, Klein MB. Elimination of HCV as a public health concern among people who inject drugs by 2030 - What will it take to get there? Journal of the International AIDS Society. 2017;20(1).

59. Schlicting EG, Johnson ME, Brems C, Wells RS, Fisher DG, Reynolds G. Validity of injecting drug users' self report of hepatitis A, B, and C. Clinical laboratory science : journal of the American Society for Medical Technology. 2003;16(2):99-106.

60. Hagan H, McGough JP, Thiede H, Hopkins SG, Weiss NS, Alexander ER. Volunteer bias in nonrandomized evaluations of the efficacy of needle-exchange programs. Journal of Urban Health. 2000;77(1):103-112. 
61. Schechter MT, Strathdee SA, Cornelisse PGA, et al. Do needle exchange programmes increase the spread of HIV among injection drug users?: an investigation of the Vancouver outbreak. AIDS. 1999;13(6):F45-F51.

62. Cooper $\mathrm{H}$, Patall EA. The relative benefits of meta-analysis conducted with individual participant data versus aggregated data. Psychological Methods. 2009;14(2):165-176.

63. Semaan S, Fleming P, Worrell C, Stolp H, Baack B, Miller M. Potential role of safer injection facilities in reducing HIV and hepatitis $\mathrm{C}$ infections and overdose mortality in the United States. Drug Alcohol Depend. 2011;118(2-3):100-110.

64. Sawangjit R, Khan TM, Chaiyakunapruk N. Effectiveness of pharmacy-based needle/syringe exchange programme for people who inject drugs: a systematic review and meta-analysis. Addiction. 2016;112(2):236-247.

65. Hagan H, Des Jarlais DC, Purchase D, Reid T, Friedman SR. The Tacoma Syringe Exchange. Journal of addictive diseases. 1991;10(4):81-88.

66. Watters JK, Estilo MJ, Clark GL, Lorvick J. Syringe and needle exchange as HIV/AIDS prevention for injection drug users. Jama. 1994;271(2):115-120.

67. Anderson W. The New York Needle Trial: the politics of public health in the age of AIDS. Am J Public Health. 1991;81(11):1506-1517.

68. Des Jarlais DC, Nugent A, Solberg A, Feelemyer J, Mermin J, Holtzman D. Syringe Service Programs for Persons Who Inject Drugs in Urban, Suburban, and Rural Areas - United States, 2013. MMWR Morb Mortal Wkly Rep. 2015;64(48):1337-1341.

69. Bishaw A PK. A Comparison of Rural and Urban America: Household Income and Poverty. 2016. https://www.census.gov/newsroom/blogs/randomsamplings/2016/12/a comparison of rura.html. Accessed 1/28/2018.

70. Singh GK, Kogan MD, Slifkin RT. Widening Disparities In Infant Mortality And Life Expectancy Between Appalachia And The Rest Of The United States, 1990-2013. Health affairs (Project Hope). 2017;36(8):1423-1432.

71. Ulrich-Schad JD, Duncan CM. People and places left behind: work, culture and politics in the rural United States. The Journal of Peasant Studies. 2018;45(1):59-79.

72. Des Jarlais DC. Harm reduction in the USA: the research perspective and an archive to David Purchase. Harm Reduct J. 2017;14(1):51.

73. Schnabel L, Sevell E. Should Mary and Jane Be Legal?Americans' Attitudes toward Marijuana and Same-Sex Marriage Legalization, 1988-2014. Public Opinion Quarterly. 2017;81(1):157-172.

74. Nadelmann E, LaSalle L. Two steps forward, one step back: current harm reduction policy and politics in the United States. Harm Reduction Journal. 2017;14(1):37.

75. Golding NJ. The Needle and the Damage Done: Indiana's Response to the 2015 HIV Epidemic and the Need to Change State and Federal Policies regarding Needle Exchanges and Intravenous Drug Users. Indiana Health Law Review. 2017;14(2):173-220.

76. Beletsky L, Agrawal A, Moreau B, Kumar P, Weiss-Laxer N, Heimer R. Police training to align law enforcement and HIV prevention: preliminary evidence from the field. Am J Public Health. 2011;101(11):2012-2015.

77. Beletsky L, Macalino GE, Burris S. Attitudes of police officers towards syringe access, occupational needle-sticks, and drug use: A qualitative study of one city police department in the United States. International Journal of Drug Policy. 2005;16(4):267-274.

78. Cepeda JA, Strathdee SA, Arredondo J, et al. Assessing police officers' attitudes and legal knowledge on behaviors that impact HIV transmission among people who inject drugs. The International journal on drug policy. 2017;50:56-63. 
79. Davis CS, Johnston J, de Saxe Zerden L, Clark K, Castillo T, Childs R. Attitudes of North Carolina law enforcement officers toward syringe decriminalization. Drug and Alcohol Dependence. 2014;144:265-269.

80. Strathdee SA, Arredondo J, Rocha T, et al. A police education programme to integrate occupational safety and HIV prevention: protocol for a modified stepped-wedge study design with parallel prospective cohorts to assess behavioural outcomes. BMJ Open. 2015;5(8).

81. Taussig J, Junge B, Burris S, Stephen Jones T, Sterk CE. Individual and Structural Influences Shaping Pharmacists Decisions to Sell Syringes to Injection Drug Users in Atlanta, Georgia. Journal of the American Pharmaceutical Association.42(6):S40-S45.

82. Lewis BA, Koester SK, Bush TW. Pharmacists' attitudes and concerns regarding syringe sales to injection drug users in Denver, Colorado. Journal of the American Pharmaceutical Association (Washington,DC : 1996). 2002;42(6 Suppl 2):S46-51.

83. Janulis P. Pharmacy nonprescription syringe distribution and HIV/AIDS: a review. Journal of the American Pharmacists Association : JAPhA. 2012;52(6):787-797.

84. Pollini RA, Rudolph AE, Case P. Nonprescription syringe sales: a missed opportunity for HIV prevention in California. Journal of the American Pharmacists Association : JAPhA. 2015;55(1):31-40.

85. Gleghorn AA, Gee G, Vlahov D. Pharmacists' attitudes about pharmacy sale of needles/syringes and needle exchange programs in a city without needle/syringe prescription laws. Journal of acquired immune deficiency syndromes and human retrovirology : official publication of the International Retrovirology Association. 1998;18 Suppl 1:S89-93. 


\section{APPENDICES}

7.1 Study 1 Search Strategies and Results

7.2 Study 2 Interview Guides

7.3 Study 3 Survey 


\subsection{Study 1 Search Strategy and Results}

\subsubsection{PubMed Search Details}

\section{PubMed Search 07/18/2016}

\section{Result:}

$\underline{314}$

Stopword(s) Ignored:

Use

\section{Translations:}

hepatitis $\mathrm{C}$

"hepatitis c"[MeSH Terms] OR "hepatitis c"[All Fields] OR "hepacivirus"[MeSH Terms] OR "hepacivirus"[All Fields]

intravenous

"substance abuse, intravenous"[MeSH Terms] OR ("substance"[All Fields] AND "abuse"[All Fields] AND

drug abuse "intravenous"[All Fields]) OR "intravenous substance abuse"[All Fields] OR ("intravenous"[All Fields] AND "drug"[All Fields] AND "abuse"[All Fields]) OR "intravenous drug abuse"[All Fields] 


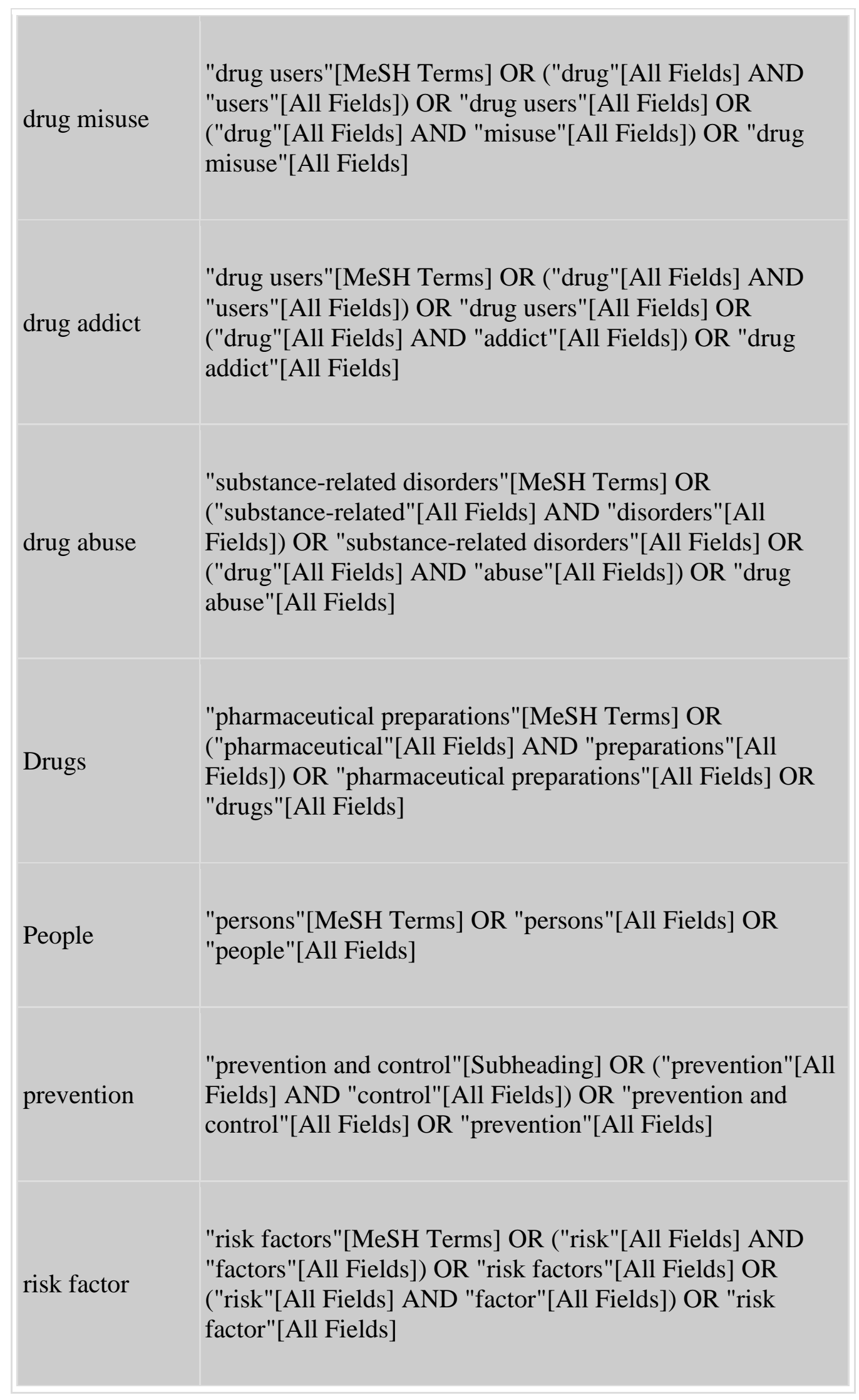


epidemiology
Fields] OR "epidemiology"[MeSH Terms]

"epidemiology"[Subheading] OR "epidemiology"[All

prevalence Fields] OR "prevalence"[All Fields] OR "prevalence"[MeSH Terms]

"epidemiology"[Subheading] OR "epidemiology"[All

incidence Fields] OR "incidence"[All Fields] OR "incidence"[MeSH

Terms]

"seroepidemiologic studies"[MeSH Terms] OR

seroprevalence

("seroepidemiologic"[All Fields] AND "studies"[All Fields])

OR "seroepidemiologic studies"[All Fields] OR

"seroprevalence"[All Fields]

seroconversion

"seroconversion"[MeSH Terms] OR "seroconversion"[All Fields]

genotype $\quad$ "genotype"[MeSH Terms] OR "genotype"[All Fields]

exchange "Sex Health Exch"[Journal] OR "exchange"[All Fields]

Needle

"needles"[MeSH Terms] OR "needles"[All Fields] OR

"needle"[All Fields]

needle

exchange

program "needle-exchange programs"[MeSH Terms] OR ("needleexchange"[All Fields] AND "programs"[All Fields]) OR "needle-exchange programs"[All Fields] OR ("needle"[All 
Fields] AND "exchange"[All Fields] AND "program"[All

Fields]) OR "needle exchange program"[All Fields]

syringe

exchange

program

Syringe "needle-exchange programs"[MeSH Terms] OR ("needleexchange"[All Fields] AND "programs"[All Fields]) OR "needle-exchange programs"[All Fields] OR ("syringe"[All Fields] AND "exchange"[All Fields] AND "program"[All Fields]) OR "syringe exchange program"[All Fields]

"syringes"[MeSH Terms] OR "syringes"[All Fields] OR "syringe"[All Fields]

\section{Database:}

\section{PubMed}

\section{User query:}

(hepatitis C OR HCV) AND (intravenous drug abuse OR intravenous drug use OR drug misuse OR drug addict OR injecting drug use OR drug abuse OR people who inject drugs OR IDU OR PWID) AND (prevention OR risk factor OR epidemiology OR prevalence OR incidence OR seroprevalence OR seroincidence OR seroconversion OR genotype OR coinfect ${ }^{*}$ ) AND (needle exchange OR needle exchange program OR syringe exchange program OR syringe access program) AND ("1989/01/01"[Date - Publication] : "3000"[Date - Publication]) 


\subsubsection{Scopus Search Details}

\section{Scopus Query Details 7/18/2016}

Scopus refine results values

Your query : ((TITLE-ABS-KEY("hepatitis C") OR TITLE-ABS-KEY("HCV") AND TITLE-ABS-

KEY("intravenous drug abuse") OR TITLE-ABS-KEY("intravenous drug use") OR TITLE-ABS-KEY("drug misuse") OR TITLE-ABS-KEY("drug addict") OR TITLE-ABS-KEY("injecting drug use") OR TITLE-ABSKEY("drug abuse") OR TITLE-ABS-KEY("people who inject drugs") OR TITLE-ABS-KEY("IDU") OR TITLEABS-KEY("PWID") AND TITLE-ABS-KEY("prevention") OR TITLE-ABS-KEY("risk factor") OR TITLE-ABSKEY("epidemiology") OR TITLE-ABS-KEY("prevalence") OR TITLE-ABS-KEY("incidence") OR TITLE-ABSKEY("seroprevalence") OR TITLE-ABS-KEY("seroincidence") OR TITLE-ABS-KEY("seroconversion") OR TITLE-ABS-KEY("genotype") OR TITLE-ABS-KEY("coinfect*") AND TITLE-ABS-KEY("needle exchange") OR TITLE-ABS-KEY("needle exchange program") OR TITLE-ABS-KEY("syringe exchange program") OR TITLEABS-KEY("syringe access program")) AND PUBYEAR > 1988)

Number of results : 226 


\subsubsection{Web of Science Search Details}

\section{Searched 8-18-16}

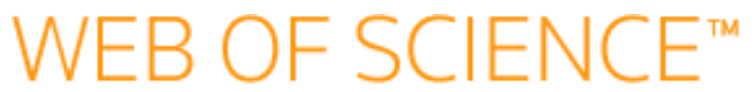

\section{THOMSON REUTERS}

\section{Search}

\section{My Tools}

\section{Search History}

-Top of Form

\section{Search History: All Databases}

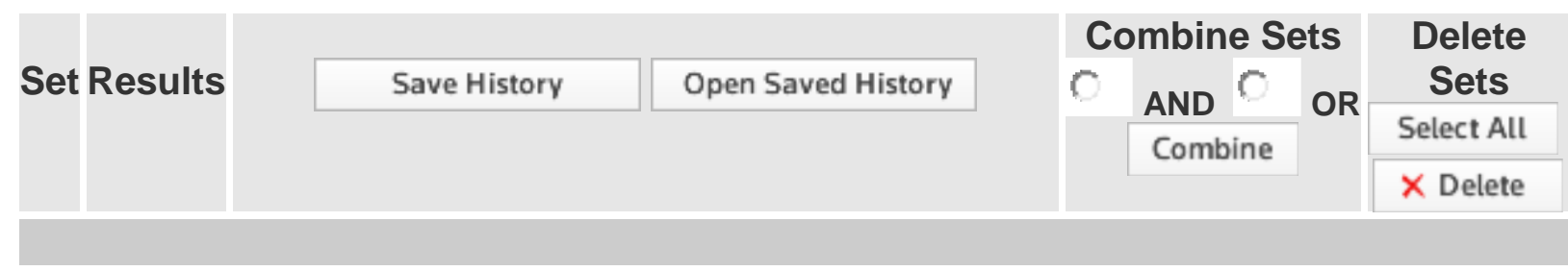

\# 1511 TOPIC: ((()hepatitis C OR HCV) AND (intravenous drug Select to combine Select to abuse OR intravenous drug use OR drug misuse OR drug addict OR injecting drug use OR drug abuse OR people who inject drugs OR IDU OR PWID) AND sets. delete this (prevention OR risk factor OR epidemiology OR set. prevalence OR incidence OR seroprevalence OR seroincidence OR seroconversion OR genotype OR coinfect $^{\star}$ ) AND (needle exchange OR needle exchange program OR syringe exchange program OR syringe access program ))))

Timespan=1989-2016

Search language= Auto

\begin{tabular}{|l|l|l|l|}
\hline Select All \\
\hline
\end{tabular} \mid \begin{tabular}{|l|l|}
\hline AND & OR \\
\hline
\end{tabular}

(C) 2016 THOMSON REUTERS 


\subsubsection{CINHAL Search Details}

CINHAL Search 7/18/16

\begin{tabular}{|c|c|c|}
\hline S1 & $\begin{array}{l}\text { (hepatitis C OR HCV) AND } \\
\text { (intravenous drug abuse OR } \\
\text { intravenous drug use OR drug } \\
\text { misuse OR drug addict OR } \\
\text { injecting drug use OR drug } \\
\text { abuse OR people who inject } \\
\text { drugs OR IDU OR PWID) AND } \\
\text { (prevention OR risk factor OR } \\
\text { epidemiology OR prevalence } \\
\text { OR incidence OR } \\
\text { seroprevalence OR } \\
\text { seroincidence OR } \\
\text { seroconversion OR genotype } \\
\text { OR coinfect*) AND (needle } \\
\text { exchange OR needle } \\
\text { exchange program OR syringe } \\
\text { exchange program OR syringe } \\
\text { access program) }\end{array}$ & Search modes - Find all my search terms \\
\hline
\end{tabular}

Number of Results: 102 


\subsection{Study 2 Interview Guides}

\subsubsection{Needle Exchange Program Directors}

Introductory Script

"Thank you again for taking the time to discuss your exchange program today. Over the next hour I will be asking you a series of questions about the past, present, and future of your program. Everything we discuss will remain confidential, and I will not identify your program by name in any publications that may result from this qualitative research study. As a reminder, participation in this study is entirely voluntary and you can choose to stop at any time without penalty. This research study is being completed as part of a dissertation for a Doctorate in Public Health degree, and has been reviewed and acknowledged by the WVU Institutional Review Board."

"Would you like to begin the interview?"

If NO: "I understand. Thank you again for letting me visit today. Have a nice day."

If YES: "Great! To help me remember everything we discuss, I would like to audio record our interview, which will be transcribed and analyzed. Would it be okay if I audio recorded our interview today?"

IF NO: "I understand. Would it be okay if I wrote some notes during our interview?"

IF YES: “Great! Let's get started. I'm going to turn the audio recorder on now.” [Turn on audio recorder]

\begin{tabular}{|l|l|}
\hline Timeframe & Guiding Questions \\
\hline Past & $\begin{array}{l}\text { 1. Can you tell me a little bit about how the program started? [Probe for precipitating events] } \\
\text { 2. Can you tell me about some of the major facilitators that helped make the program possible? [Probe for } \\
\text { champions] }\end{array}$ \\
\hline 3. Can you tell me about some of the major barriers encountered to opening the program? [Probe for \\
local/state/legal]
\end{tabular}

"Thank you sincerely for your time today. Once the data are analyzed, I would like to speak with you again regarding the results to get your thoughts. Have a nice day." 


\subsubsection{Law Enforcement}

Introductory Script

"Thank you again for taking the time to discuss your thoughts on the local exchange program today. Over the next hour I will be asking you a series of questions about the past, present, and future of the program. Everything we discuss will remain confidential, and I will not identify you by name in any publications that may result from this qualitative research study. As a reminder, participation in this study is entirely voluntary and you can choose to stop at any time without penalty. This research study is being completed as part of a dissertation for a Doctorate in Public Health degree, and has been reviewed and acknowledged by the WVU Institutional Review Board."

"Would you like to begin the interview?"

If NO: "I understand. Thank you again for letting me visit today. Have a nice day."

If YES: "Great! To help me remember everything we discuss, I would like to audio record our interview, which will be transcribed and analyzed. Would it be okay if I audio recorded our interview today?"

IF NO: "I understand. Would it be okay if I wrote some notes during our interview?"

IF YES: “Great! Let's get started. I'm going to turn the audio recorder on now.” [Turn on audio recorder]

\begin{tabular}{|c|c|}
\hline Timeframe & Guiding Questions \\
\hline Past & $\begin{array}{l}\text { 16. Can you tell me a little bit about how the program started? [Probe for precipitating events] } \\
\text { 17. Can you tell me about some of the major facilitators that helped make the program possible? [Probe for } \\
\text { champions] } \\
\text { 18. Can you tell me about some of the major barriers encountered to opening the program? [Probe for } \\
\text { local/state/legal] }\end{array}$ \\
\hline Present & $\begin{array}{l}\text { 19. "How would you characterize the program's current relationship with the police department?" } \\
\text { 20. "Can you tell me a little bit about the impact of the program on law enforcement? [Probe for dirty } \\
\text { needles/confiscation of clean needles/perceived impact on drug use/perceived impact on drug related } \\
\text { problems/crime] } \\
\text { 21. "Can you discuss the ways in which the program has impacted your daily job?" } \\
\text { 22. Can you tell me a little bit more about your feelings regarding the program's location [Probe for proximity to } \\
\text { the police station] } \\
\text { 23. "Can you tell me a little bit about the program's attendees?" } \\
\text { 24. "Can you tell me a little bit about any current difficulties related to the program?" [Probe for drug } \\
\text { paraphernalia laws/political environment/opponents/naloxone availability/overdose concerns] } \\
\text { 25. "Can you tell me a little bit about the things that are helpful to the program?" For example, what is } \\
\text { something that you don't think the program could function optimally without?" } \\
\text { 26. "What are the surrounding community's feelings about the program?" }\end{array}$ \\
\hline Future & $\begin{array}{l}\text { 27. What, if anything, would you change about the current program? } \\
\text { 28. What would you like this program to look like in five years? [Probe for barriers to realizing future goals] }\end{array}$ \\
\hline
\end{tabular}




\title{
7.2.3 Needle Exchange Program Attendees
}

\author{
Introductory Script
}

"Thank you again for taking the time to discuss your experiences with the exchange program and your opinions on clean syringe use today. My name is Steve Davis, and I am with the WVU Department of Emergency Medicine. This research study is being completed as part of a dissertation for a Doctorate in Public Health degree, and has been reviewed and acknowledged by the WVU Institutional Review Board. If you have any questions about this research, you can contact me at 304-293-1326 or via my email at smdavis@hsc.wvu.edu. "

Over the next 30 minutes I will be asking you a series of questions about your experiences with the exchange and your opinions about an anonymous survey on barriers to using clean syringes (or needles). We plan to administer this survey to fellow attendees in the near future. Your responses will help us understand if the survey makes sense and if we have potentially left out any important barriers to clean syringe use. Everything we discuss will remain confidential, and I will not identify you by name in any publications or reports that may result from this research study. As a reminder, participation in this study is entirely voluntary and you can choose to skip any question and stop at any time without penalty.

"Would you like to begin the interview?"

If NO: "I understand. Thank you again for letting me visit today. Have a nice day."

If YES: "Great! To help me remember everything we discuss, I would like to audio record our interview, which will be transcribed and analyzed. Would it be okay if I audio recorded our interview today?"

IF NO: "I understand. Would it be okay if I wrote some notes during our interview?"

IF YES: “Great! Please do not give your name or any other identifying information during our discussions, and please do not share anything we discuss today outside of this interview. Also, please do not record your individual responses to the Barriers to Using New Needles survey. Let's get started. I'm going to turn the audio recorder on now." [Turn on audio recorder]

\begin{tabular}{|l|l|}
\hline PART 1 (TRIANGULATION) & Guiding Questions \\
\hline & $\begin{array}{l}\text { 29. What do you call this place? } \\
\text { 30. Can you tell me a little bit about what it was like for attendees before the exchange opened? } \\
\text { \{PAST } \\
\text { 31. Can you tell me a little bit more about the current need for the program? [probe for increasing } \\
\text { volumes or volume changes/infectious disease prevention/linkage to treatment] \{PRESENT\} } \\
\text { 32. Can you tell me a little bit about any current difficulties related to using the exchange?" [probe } \\
\text { for transportation barriers, location barriers, legal barriers (i.e., fear of arrest), community } \\
\text { opposition, wait times that may be related to increasing volumes)] \{PRESENT\} } \\
\text { 33. Can you tell me a little bit about the things that are helpful to operating the program?" For } \\
\text { example, what is something that you don't think the program could function optimally } \\
\text { without?" [probe for community/people champions/funding] \{PRESENT\} } \\
\text { 34. Dream big! What changes would you like to see in the program in } 1 \text { year? } 5 \text { years? [probe for } \\
\text { barriers to realizing future goals] \{FUTURE\} }\end{array}$ \\
\hline
\end{tabular}




\begin{tabular}{|l|l|}
\hline $\begin{array}{l}\text { PART 2 (SURVEY } \\
\text { COMPREHENSION) }\end{array}$ & Guiding Questions \\
\hline $\begin{array}{l}\text { Give participants the } \\
\text { Barriers to Using New } \\
\begin{array}{l}\text { Needles survey and have } \\
\text { them complete the } \\
\text { questionnaire but NOT } \\
\text { record their actual results. }\end{array}\end{array}$ & $\begin{array}{l}\text { 1. } \\
\text { Understandable: Were the items "understandable"? That is, were you able to read the } \\
\text { questions once and understand what it was they were asking? Were the meanings of the } \\
\text { questions clear and straightforward? }\end{array}$ \\
$\begin{array}{l}\text { Note the time taken to } \\
\text { complete the } \\
\text { questionnaire. }\end{array}$ & $\begin{array}{l}\text { That is, do you feel the choices provided you with an appropriate way to respond? } \\
\text { Only One Response: Were the items written in such a way that you could have answered in } \\
\text { only one way (i.e., you did not wish to answer BOTH "strongly agree" and "strongly disagree"). } \\
\text { Complete: Were there any other barriers to using a clean syringe that you feel were not } \\
\text { included in the items? If so, what are they? }\end{array}$ \\
$\begin{array}{l}\text { Once finished, ask the guide } \\
\text { questions }\end{array}$ & \begin{tabular}{l} 
\\
\hline
\end{tabular}
\end{tabular}

"Thank you sincerely for your time today. Once the data are analyzed, I would like to speak with you again regarding the results to get your thoughts. Have a nice day." 


\subsection{Study 3 Barriers to Using New Needles Survey Part I. For each question, please circle only one (1) response.}

1. It takes too long to get a new needle every time I inject.

2. It is inconvenient to get a new needle every time I inject.

3. I often don't want to take the time to get a new needle because my cravings or urges to use drugs are too strong.

4. I often do not take the time to get a new needle if I am drug sick or in withdrawal.

5. I don't take the time to get a new needle before injecting because I can only think about getting high.

6. I don't take the time to get a new needle before injecting if I'm already high or drunk.

7. The places where I inject usually do not have access to new needles.

8. If I am in a shooting gallery (a place where people inject drugs, "dope den", "joy popping", etc.), I often do not use a new needle.

9. I often do not carry new needles with me when I'm out.

10. There isn't a needle exchange close by for me to get needles.

11. Pharmacies sometimes give me hassle when I try to buy needles.

12. After I inject, I don't prepare in advance by getting new needles ready for my next injection.

13. It's too expensive to buy new needles from the pharmacy for every time I inject.

14. Feeling sad or depressed would get in the way of my using a new needle every time I inject.

15. It is embarrassing to buy needles at the pharmacy.

16. I worry that someone (friends, family, etc.) may see me buying needles at the pharmacy.

17. My peers/friends would look at me funny if I used a new needle every time I inject.

18. Having to worry about using a new needle interrupts the ritual of using.

19. I am unlikely to use a new needle if a friend lets me borrow his or her used needle.

20. I could get in trouble from the police if I carry needles around with me.

\begin{tabular}{|c|c|c|c|c|c|}
\hline 1 & 2 & 3 & 4 & 5 & \\
\hline $\begin{array}{l}\text { Strongly } \\
\text { Disagree }\end{array}$ & Disagree & Neutral & Agree & $\begin{array}{c}\text { Strongly } \\
\text { Agree }\end{array}$ & $\begin{array}{c}\text { Not } \\
\text { Applicable }\end{array}$ \\
\hline $\begin{array}{l}\text { Strongly } \\
\text { Disagree }\end{array}$ & Disagree & Neutral & Agree & $\begin{array}{c}\text { Strongly } \\
\text { Agree }\end{array}$ & $\begin{array}{c}\text { Not } \\
\text { Applicable }\end{array}$ \\
\hline $\begin{array}{l}\text { Strongly } \\
\text { Disagree }\end{array}$ & Disagree & Neutral & Agree & $\begin{array}{c}\text { Strongly } \\
\text { Agree }\end{array}$ & $\begin{array}{c}\text { Not } \\
\text { Applicable }\end{array}$ \\
\hline $\begin{array}{l}\text { Strongly } \\
\text { Disagree }\end{array}$ & Disagree & Neutral & Agree & $\begin{array}{c}\text { Strongly } \\
\text { Agree }\end{array}$ & $\begin{array}{c}\text { Not } \\
\text { Applicable }\end{array}$ \\
\hline $\begin{array}{l}\text { Strongly } \\
\text { Disagree }\end{array}$ & Disagree & Neutral & Agree & $\begin{array}{c}\text { Strongly } \\
\text { Agree }\end{array}$ & $\begin{array}{c}\text { Not } \\
\text { Applicable }\end{array}$ \\
\hline $\begin{array}{l}\text { Strongly } \\
\text { Disagree }\end{array}$ & Disagree & Neutral & Agree & $\begin{array}{c}\text { Strongly } \\
\text { Agree }\end{array}$ & $\begin{array}{c}\text { Not } \\
\text { Applicable }\end{array}$ \\
\hline $\begin{array}{l}\text { Strongly } \\
\text { Disagree }\end{array}$ & Disagree & Neutral & Agree & $\begin{array}{c}\text { Strongly } \\
\text { Agree }\end{array}$ & $\begin{array}{c}\text { Not } \\
\text { Applicable }\end{array}$ \\
\hline $\begin{array}{l}\text { Strongly } \\
\text { Disagree }\end{array}$ & Disagree & Neutral & Agree & $\begin{array}{c}\text { Strongly } \\
\text { Agree }\end{array}$ & $\begin{array}{c}\text { Not } \\
\text { Applicable }\end{array}$ \\
\hline $\begin{array}{l}\text { Strongly } \\
\text { Disagree }\end{array}$ & Disagree & Neutral & Agree & $\begin{array}{c}\text { Strongly } \\
\text { Agree }\end{array}$ & $\begin{array}{c}\text { Not } \\
\text { Applicable }\end{array}$ \\
\hline $\begin{array}{l}\text { Strongly } \\
\text { Disagree }\end{array}$ & Disagree & Neutral & Agree & $\begin{array}{c}\text { Strongly } \\
\text { Agree }\end{array}$ & $\begin{array}{c}\text { Not } \\
\text { Applicable }\end{array}$ \\
\hline $\begin{array}{l}\text { Strongly } \\
\text { Disagree }\end{array}$ & Disagree & Neutral & Agree & $\begin{array}{c}\text { Strongly } \\
\text { Agree }\end{array}$ & $\begin{array}{c}\text { Not } \\
\text { Applicable }\end{array}$ \\
\hline $\begin{array}{l}\text { Strongly } \\
\text { Disagree }\end{array}$ & Disagree & Neutral & Agree & $\begin{array}{c}\text { Strongly } \\
\text { Agree }\end{array}$ & $\begin{array}{c}\text { Not } \\
\text { Applicable }\end{array}$ \\
\hline $\begin{array}{l}\text { Strongly } \\
\text { Disagree }\end{array}$ & Disagree & Neutral & Agree & $\begin{array}{c}\text { Strongly } \\
\text { Agree }\end{array}$ & $\begin{array}{c}\text { Not } \\
\text { Applicable }\end{array}$ \\
\hline $\begin{array}{l}\text { Strongly } \\
\text { Disagree }\end{array}$ & Disagree & Neutral & Agree & $\begin{array}{c}\text { Strongly } \\
\text { Agree }\end{array}$ & $\begin{array}{c}\text { Not } \\
\text { Applicable }\end{array}$ \\
\hline $\begin{array}{l}\text { Strongly } \\
\text { Disagree }\end{array}$ & Disagree & Neutral & Agree & $\begin{array}{c}\text { Strongly } \\
\text { Agree }\end{array}$ & $\begin{array}{c}\text { Not } \\
\text { Applicable }\end{array}$ \\
\hline $\begin{array}{l}\text { Strongly } \\
\text { Disagree }\end{array}$ & Disagree & Neutral & Agree & $\begin{array}{c}\text { Strongly } \\
\text { Agree }\end{array}$ & $\begin{array}{c}\text { Not } \\
\text { Applicable }\end{array}$ \\
\hline $\begin{array}{l}\text { Strongly } \\
\text { Disagree }\end{array}$ & Disagree & Neutral & Agree & $\begin{array}{c}\text { Strongly } \\
\text { Agree }\end{array}$ & $\begin{array}{c}\text { Not } \\
\text { Applicable }\end{array}$ \\
\hline $\begin{array}{l}\text { Strongly } \\
\text { Disagree }\end{array}$ & Disagree & Neutral & Agree & $\begin{array}{c}\text { Strongly } \\
\text { Agree }\end{array}$ & $\begin{array}{c}\text { Not } \\
\text { Applicable }\end{array}$ \\
\hline $\begin{array}{l}\text { Strongly } \\
\text { Disagree }\end{array}$ & Disagree & Neutral & Agree & $\begin{array}{c}\text { Strongly } \\
\text { Agree }\end{array}$ & $\begin{array}{c}\text { Not } \\
\text { Applicable }\end{array}$ \\
\hline $\begin{array}{l}\text { Strongly } \\
\text { Disagree }\end{array}$ & Disagree & Neutral & Agree & $\begin{array}{c}\text { Strongly } \\
\text { Agree }\end{array}$ & $\begin{array}{c}\text { Not } \\
\text { Applicable }\end{array}$ \\
\hline
\end{tabular}




\section{PART II. For the following questions, please answer or check $(\checkmark)$ the appropriate response.}

21. What is your age? (in years)

22. What is your sex?
口 Female
口 Male

23. What is the highest grade or year of school you completed?*
Never attended school or only attended kindergarten
Elementary
Some high school
- High School graduate (or GED)
Some college or technical school
- College graduate
- Some graduate or professional school
G Graduate or professional degree (for example, MS, PhD, MD)

24. How would you describe your race? (Check all that apply)
$\square$ White
Black or African American
Asian
- Native Hawaiian or Pacific Islander
- American Indian or Alaska Native
口 Other:

25. Are you Hispanic or Latino (if female, Latina)?*
Y Yes
- No

* Adapted from: CDC. 2014 Behavioral Risk Factor Surveillance System Questionnaire. 2013; https://www.cdc.gov/brfss/questionnaires/pdfques/2014 BRFSS.pdf. Accessed March 5th, 2015.
26. What is your current employment status (check only one)?*
Employed for wages
Self-employed
Out of work for more than 1 year
O Out of work for less than 1 year
A A Homemaker
A Student
$\square$ Retired
u Unable to work

27. Are you currently homeless?
$\square$ Yes
$\square$ No

28. How many times do you usually inject each day? (number of injections)

29. How many times do you usually inject each week? (number of injections)

30. How long have you been injecting? OR (if less than 1 year) years months

31. What is your drug of choice (i.e., what drug do you most often inject)?

32. Are you hepatitis $C$ positive?
$\square$ Yes
No
Don't Know

THANK YOU! PLEASE RETURN THE COMPLETED SURVEY TO MR. DAVIS AND RECEIVE YOUR \$10 SHEETZ GIFT CARD TODAY. 